\title{
Rooftop Unit Comparison Calculator User Manual
}

\author{
J.D. Miller
}

April 2015

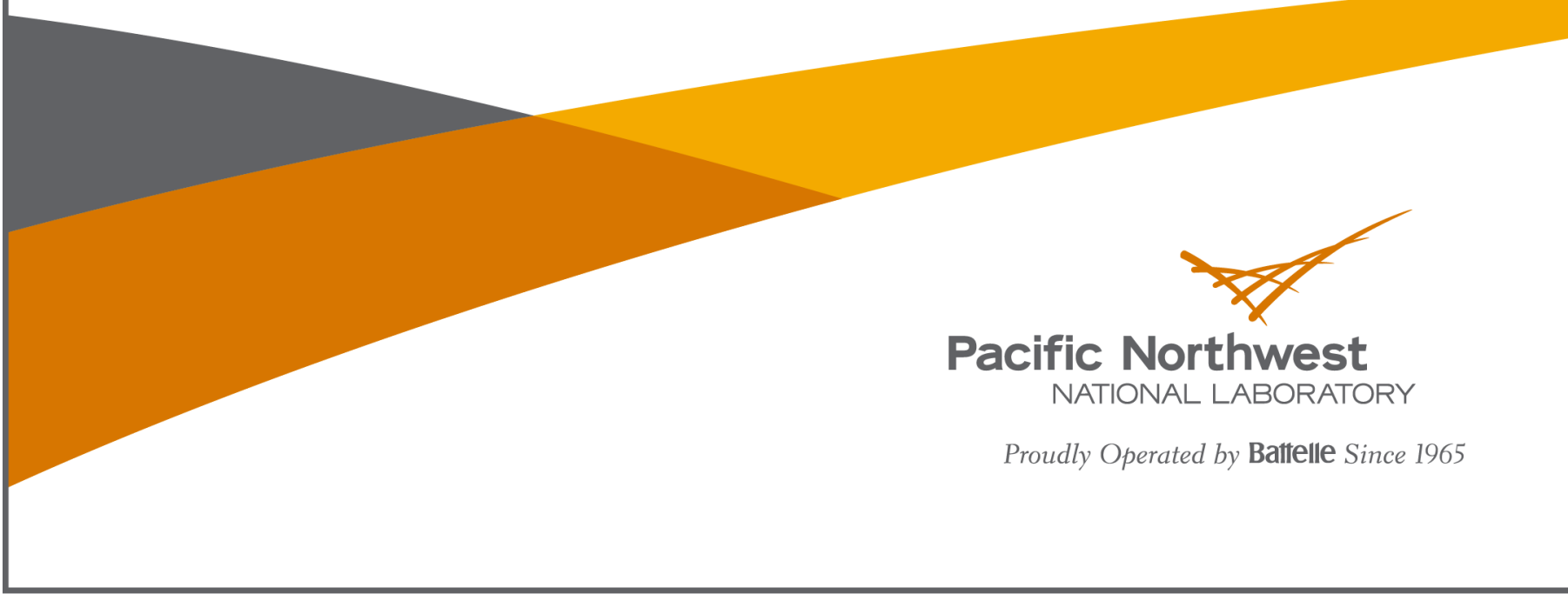




\section{DISCLAIMER}

United States Government. Neither the United States Government nor any agency thereof, nor Battelle Memorial Institute, nor any of their employees, makes any warranty, express or implied, or assumes any legal liability or responsibility for the accuracy, completeness, or usefulness of any information, apparatus, product, or process disclosed, or represents that its use would not infringe privately owned rights. Reference herein to any specific commercial product, process, or service by trade name, trademark, manufacturer, or otherwise does not necessarily constitute or imply its endorsement, recommendation, or favoring by the United States Government or any agency thereof, or Battelle Memorial Institute. The views and opinions of authors expressed herein do not necessarily state or reflect those of the United States Government or any agency thereof.

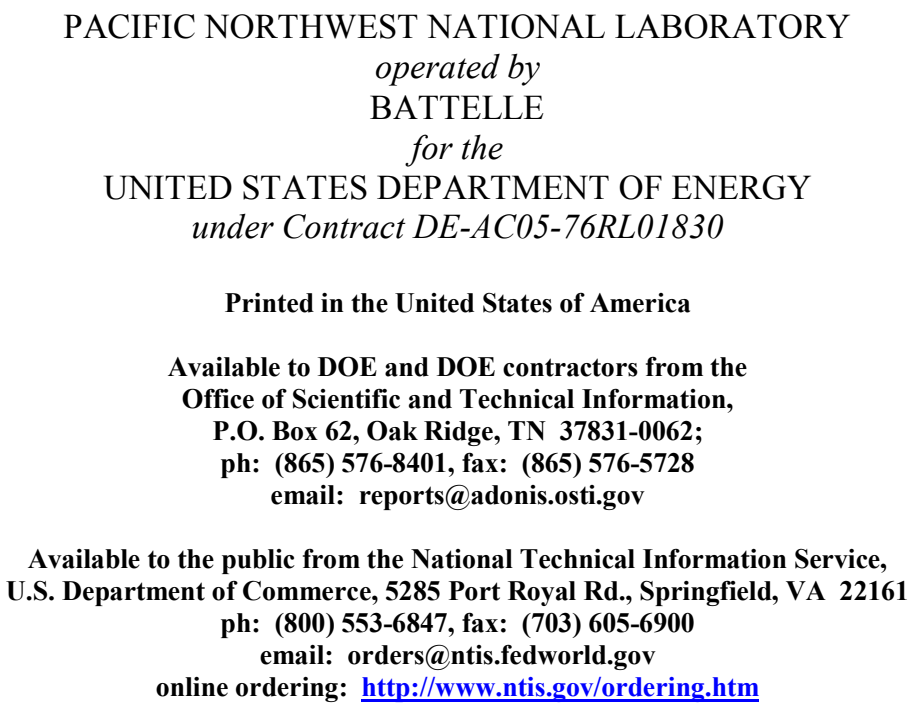

This document was printed on recycled paper. 
PNNL-24130

\section{Rooftop Unit Comparison Calculator User Manual}

J.D. Miller

April 2015

Prepared for

U.S. Department of Energy under Contract DE-AC05-76RL01830

Pacific Northwest National Laboratory

Richland, Washington 99352 


\section{SUMMARY}

Packaged rooftop air conditioners and heat pump units (RTUs) are used in 46\% (2.1 million) of all commercial buildings, serving over $60 \%$ (39 billion square feet) of the commercial building floor space in the U.S. (EIA 2003). The annual primary (source) energy consumption associated with these units is over 1.3 quads. Therefore, even a small improvement in rated efficiency or part-load operation of these units can lead to significant reductions of energy use and carbon emissions.

The majority of building owners are not familiar with the savings that the new high efficiency RTU products provide.

The Building Technologies Office (BTO) has previously funded development of the RTU comparison calculator (RTUCC). RTUCC is a web-based tool that provides the user a way to compare energy consumption and costs for two units with different efficiencies.

Prior to development work in 2014, the RTUCC could not estimate savings associated with either the RTU Challenge unit or the advanced RTU controls retrofit. Therefore, BTO funded PNNL to enhance the tool so that building owners can compare energy and savings associated with these two new classes of products. These enhancements to the calculator (version 4.3) are described in this document and are generally referred to as the "Specific Candidate Unit" feature. This document serves as a user manual for the RTUCC and is an aggregation of the calculator's website documentation. Content ranges from new-user guide material like the "Quick Start" to the more technical/algorithmic descriptions of the Methods pages. There is also a section listing all the context-help topics that support the features on the Controls page. Appendix A has a discussion of the EnergyPlus runs that supported the development of the building-response models. 


\section{ACKNOWLEDGEMENT}

The author would like to acknowledge the Building Technologies Office of the U.S. Department of Energy (DOE) Office of Energy Efficiency and Renewable Energy for supporting this effort.

The author would also like to thank Jeff McCullough whose spreadsheet prototype was the conceptual spark for this development effort, Tim Hillman who along with Jeff helped with the original design of the web interface, Todd Taylor for providing the schedule-indexed weather data, Jim McIntyre for supporting us through the many changes on the PNNL public server, Brad Hollomon for encouraging rigor in the calculation engine and supporting the development of the spreadsheet interface, David Winiarski for technical help and various discussions along the way, Peter Armstrong for supporting the first application of the calculator in evaluating specific candidate RTUs, Gregory Sullivan for his thorough technical review and his use of the calculator in commercial applications, Linda Sandahl (and Jeff) for reigniting development efforts to better represent variable-capacity condensers and variable-speed fans, Anne Wagner for her patient counsel during the external review, Michael Rosenberg for his support in using EnergyPlus to develop the building-response models, and Weimin Wang and Srinivas Katipamula for their guidance and review during the development of the "Specific Candidate Unit" feature and the computation-engine update that supported it. 


\section{Contents}

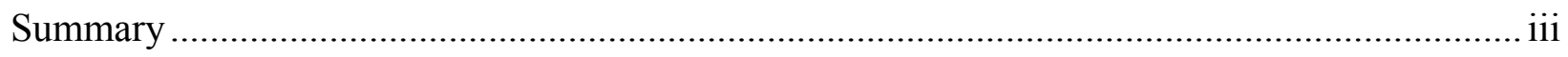

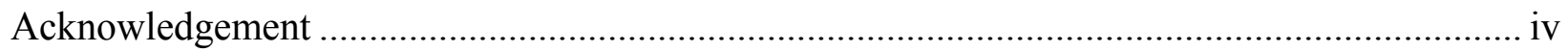

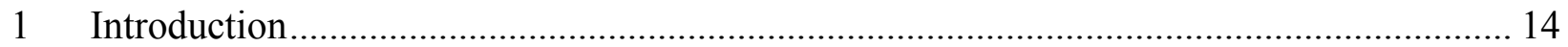

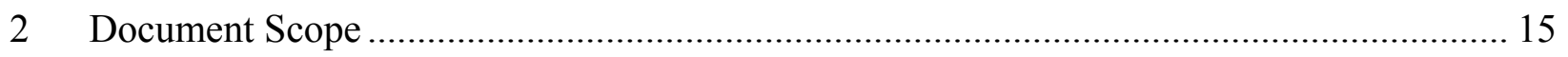

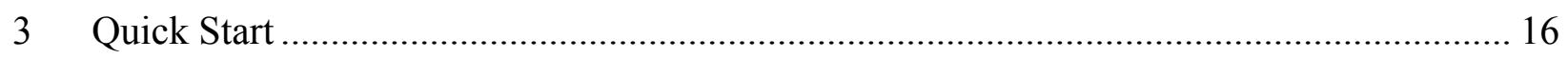

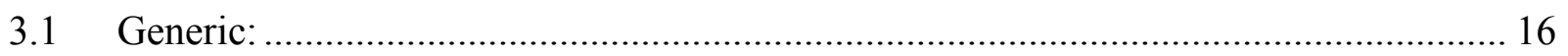

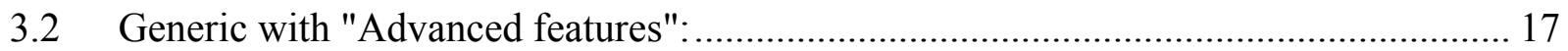

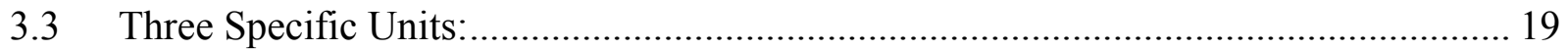

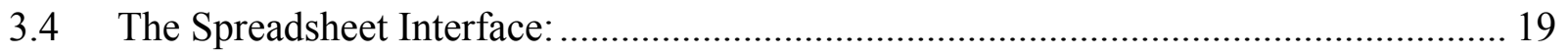

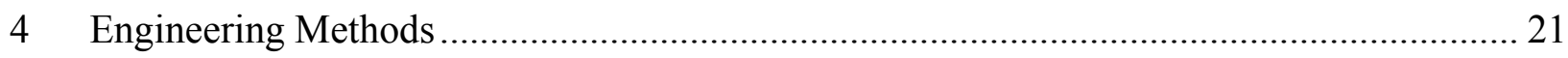

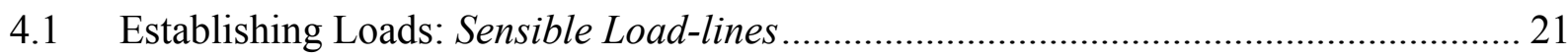

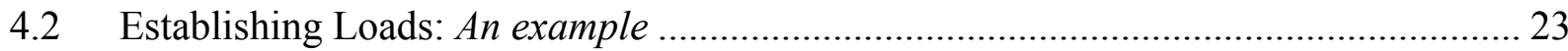

4.3 Corrections to Tested Performance: Capacity and Condenser Power ........................... 24

4.4 Corrections to Tested Performance: Sensible Capacity ..................................................... 26

4.5 Corrections to Tested Performance: the Spreadsheet Interface ..................................... 27

4.6 Corrections to Tested Performance: Three Specific RTUs ............................................ 30

4.7 Equipment Response To Loads .............................................................................. 32

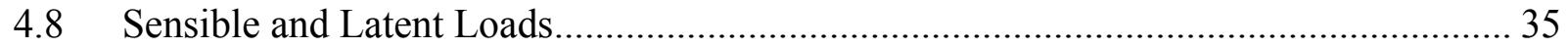

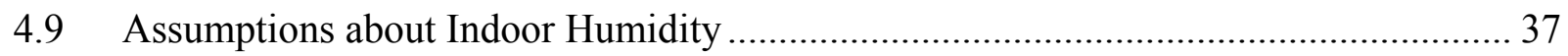

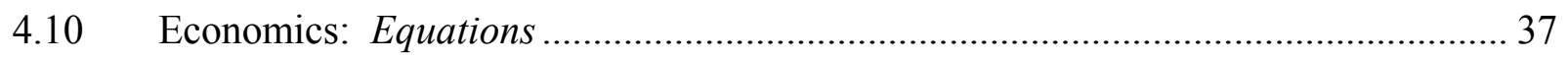

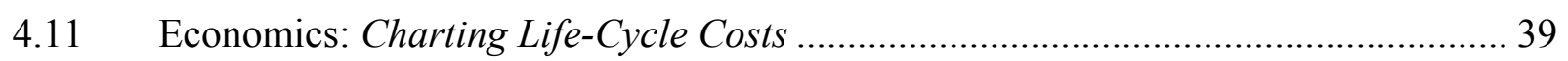

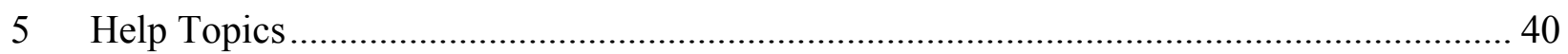

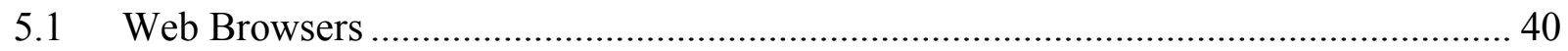

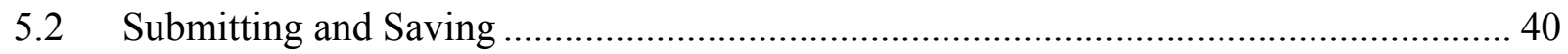

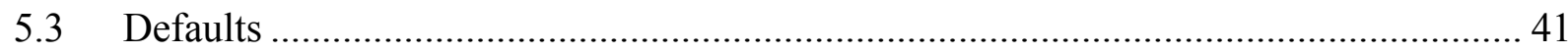

5.4 Building Type

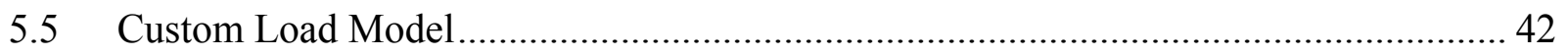

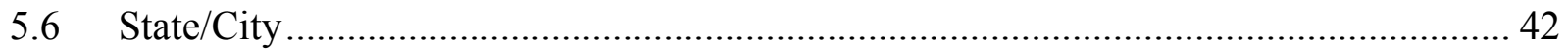

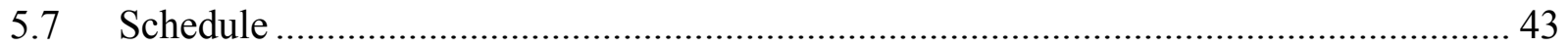

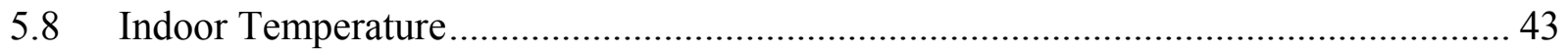

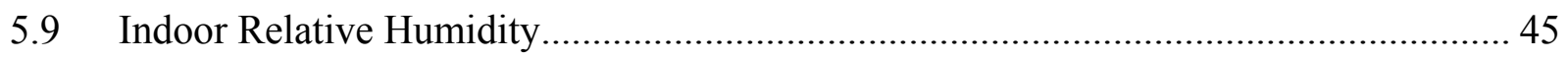




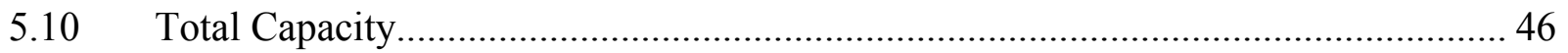

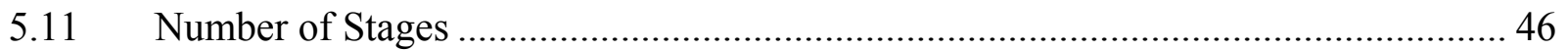

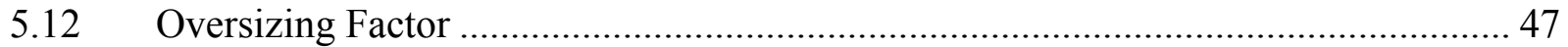

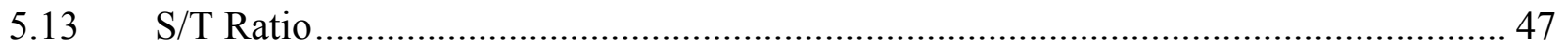

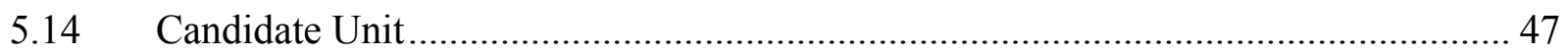

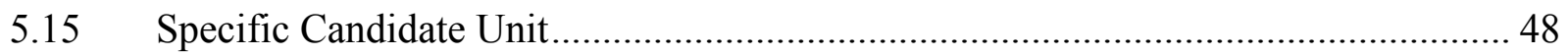

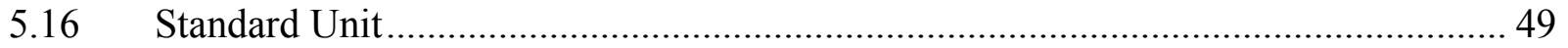

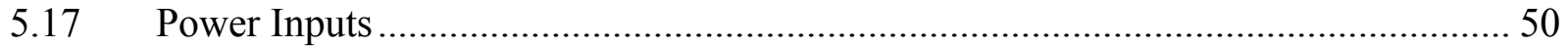

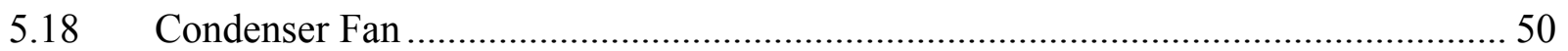

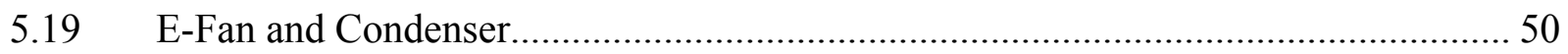

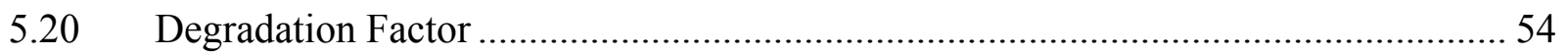

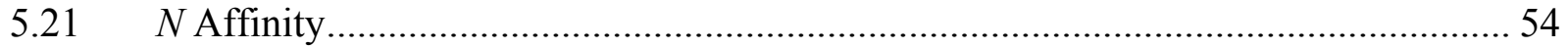

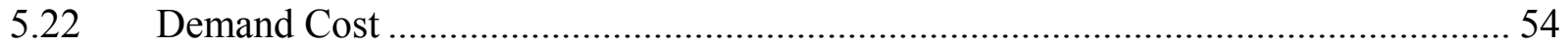

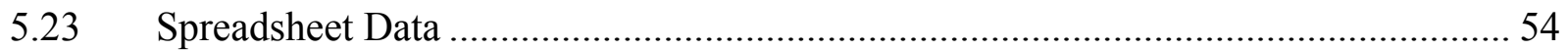

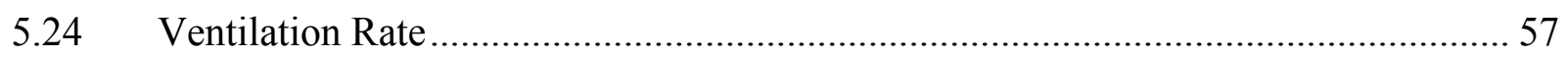

5.24.1 Effect of location (city/state) on calculated ventilation levels..............................5 58

5.24.2 Impact of ventilation changes as influenced by competing effects ....................... 58

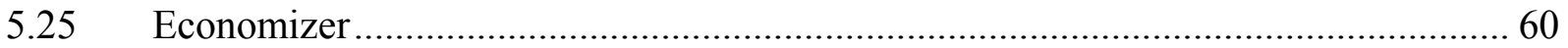

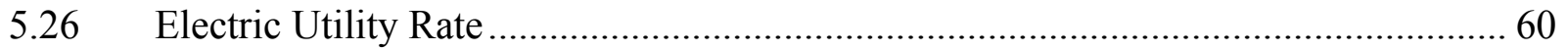

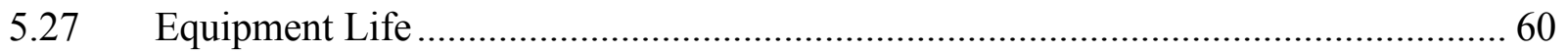

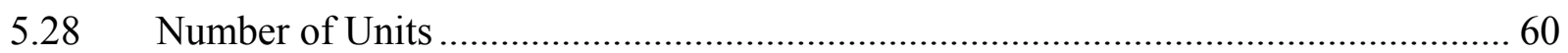

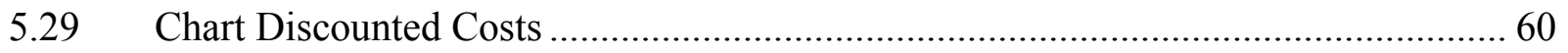

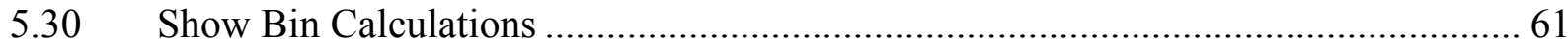

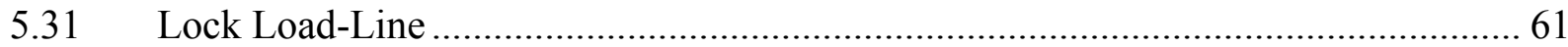

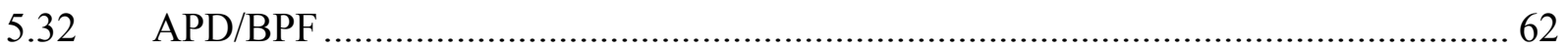

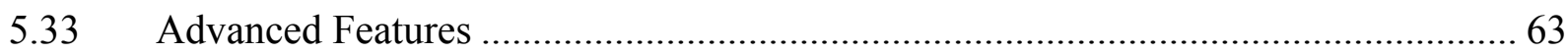

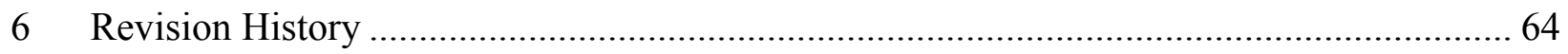

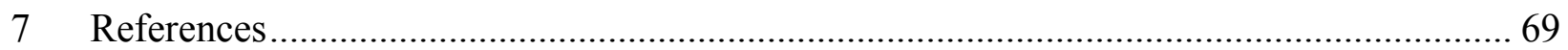

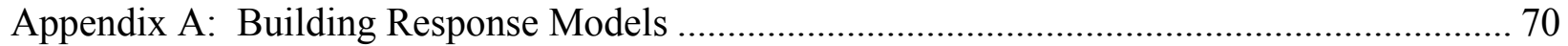

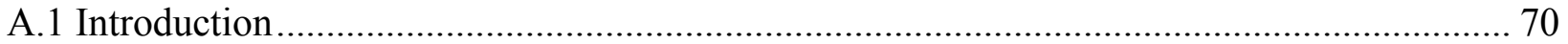

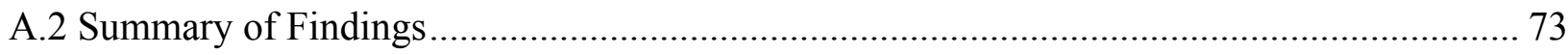

A.3 EnergyPlus Output and Post-Processing Approach................................................................ 74 


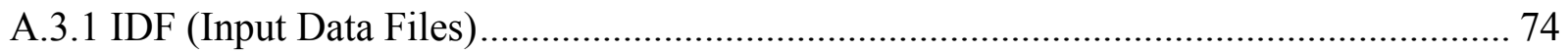

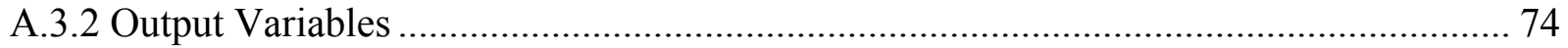

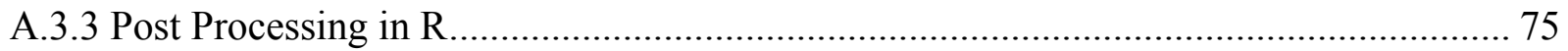

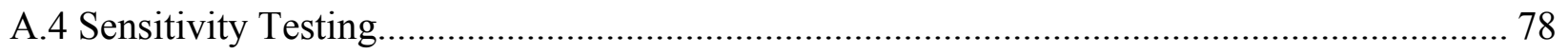

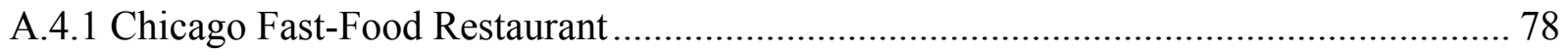

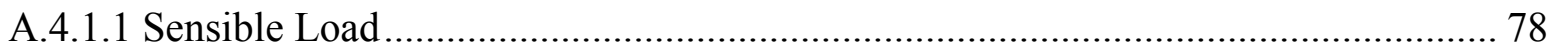

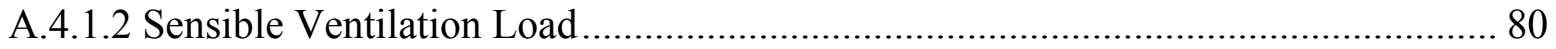

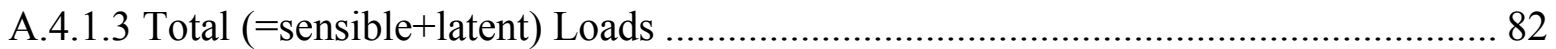

A.4.2 Chicago Medium Office / Response Models and Weather Data .................................... 83

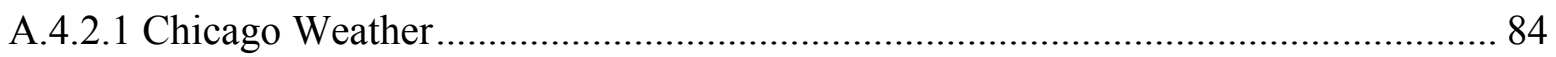

A.4.2.2 Phoenix Weather .............................................................................................. 90

A.4.2.3 San Francisco Weather....................................................................................... 91

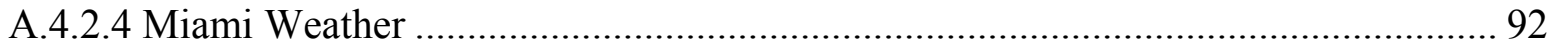

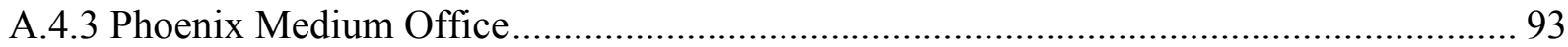

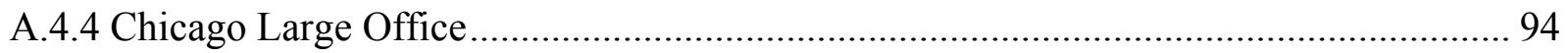

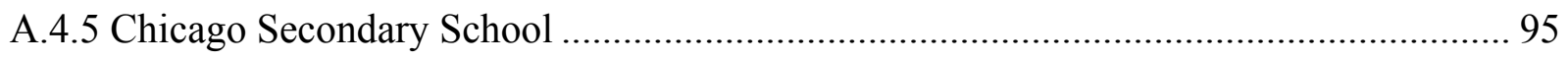

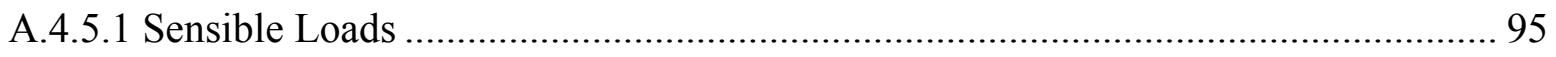

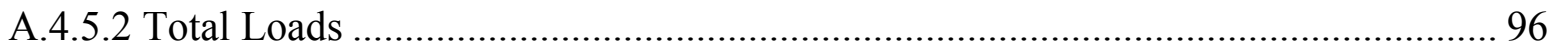

A.5 Model Runs with Chicago Weather ................................................................................ 97

A.5.1 Chicago Small Office................................................................................................ 97

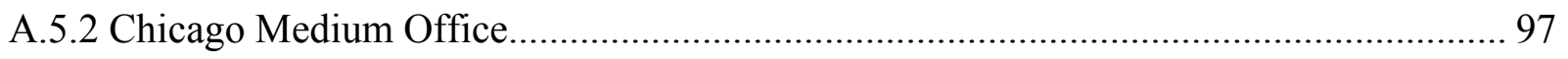

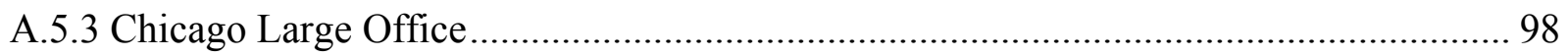

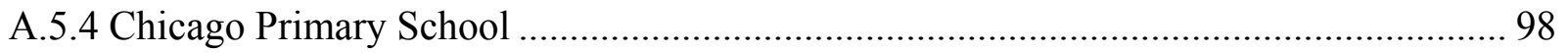

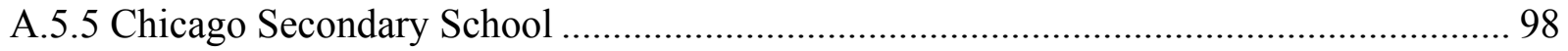

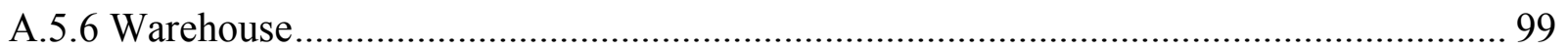

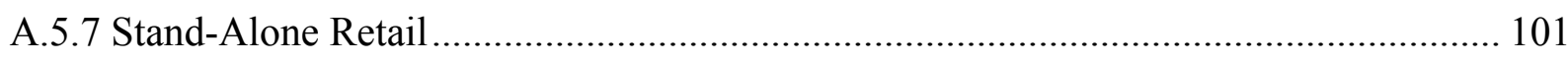

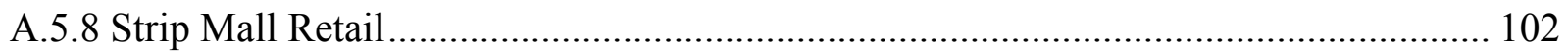

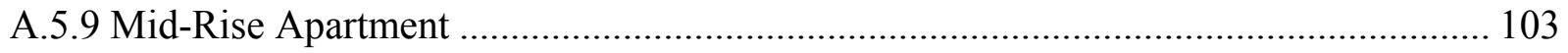

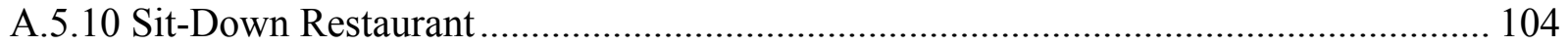

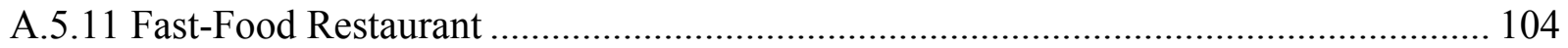

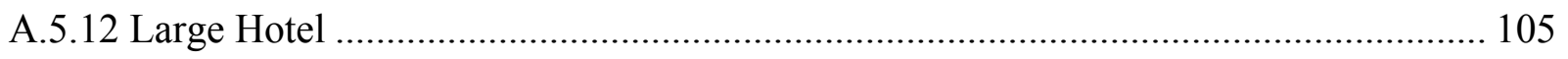

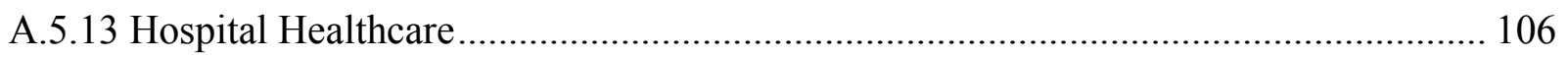




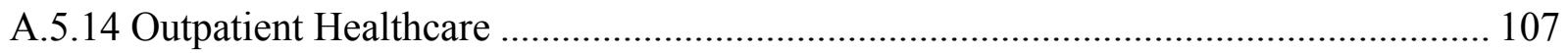

A.6 Check of Total Ventilation Load Calculation .............................................................. 108

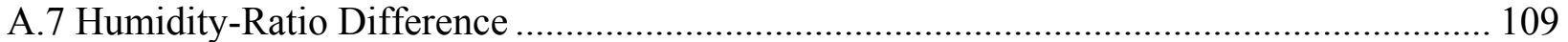




\section{Figures}

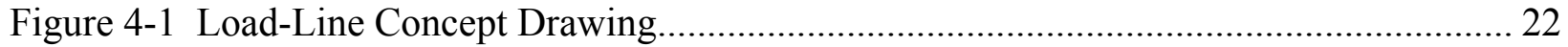

Figure 4-2 Loads and Hours: Wichita, KS; Medium Office ....................................................... 23

Figure 4-3 Illustration of DOE-2 Correction Curves (entering wet-bulb temperature $=67^{\circ} \mathrm{F}$ ) $\ldots 25$

Figure 4-4 Left: Graphical illustration of the definition of the bypass factor: ratio of segment yellow-blue to segment red-blue. Right: Graphical illustration of the iterative technique used to determine the sensible-to-total ratio from a given bypass factor and total capacity.

Figure 4-5 Total and sensible capacity data normalized by corresponding values at AHRI rating conditions. This graph shows the stronger dependence of the sensible capacity on

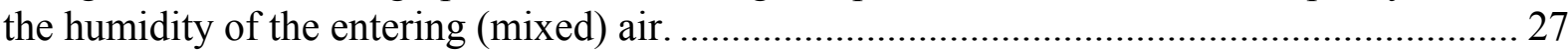

Figure 4-6 Example of performance-correction relationships for total capacity......................... 28

Figure 4-7 Examples of performance-correction relationships for power.................................... 29

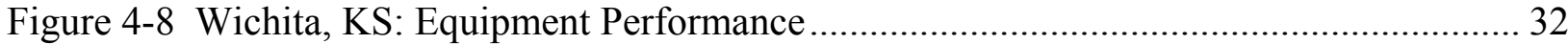

Figure 4-9 Humidity-ratio differential (outdoor minus indoor) as affected by temperature differential (outdoor minus indoor). Left: raw hourly data. Right: daily averages.................. 37

Figure 4-10 Example chart of discounted costs (Wichita, KS) .................................................. 39

Figure A-1 Sensible load as affected by envelope temperature differential. Left: Load-line concept drawing. Right: Corresponding example of load data from an EnergyPlus simulation.

Figure A-2 Sensible cooling loads as affected by envelope temperature differential. Left: DX coil loads. Right: Aggregate of zone loads

Figure A-3 Daily average sensible cooling loads for Chicago Medium Office as affected by envelope temperature differential.

Figure A-4 Raw sensible loads for Chicago Fast-Food Restaurant as affected by envelope temperature differential. Left: sensible load (Intercept $=45.8+/-0.2 \mathrm{MJ} / \mathrm{h}$; Slope $=9.97$ $+/-0.07 \mathrm{MJ} / \mathrm{hC}$ ). Right: sensible ventilation load $($ Slope $=7.61+/-0.03$; Slope Fraction $=$ 7.61/9.97 $=0.76)$.

Figure A-5 Sensible ventilation load for Kitchen and Dining zones as affected by envelope temperature differential. Left: filtered to exclude negative loads; Slope $=6.8$. Right: raw data.

Figure A-6 Sensible ventilation load for Dining zone only. Left: filtered to exclude negative loads; Slope $=1.8$. Right: raw data.

Figure A-7 Sensible ventilation load for Kitchen zone only. Left: filtered to exclude negative loads; Slope $=5.0$. Right: raw data.

Figure A-8 Raw total (latent + sensible) load for Chicago Fast-Food Restaurant as affected by envelope temperature differential. Left: total load $($ Intercept $=76.28 \mathrm{MJ} / \mathrm{h}$; Slope $=$ 
16.16 MJ/hC). Right: total ventilation load (Slope $=11.31$; Slope Fraction $=11.31 / 16.16$ $=0.70)$.

Figure A-9 Chicago Medium Office with Chicago weather. Raw sensible load as affected by envelope temperature differential. Left: sensible load (Intercept $=382+/-2 \mathrm{MJ} / \mathrm{h}$; Slope $=33.7+/-0.5 \mathrm{MJ} / \mathrm{hC}$ ). Right: sensible ventilation load (Slope = 16.91 +/- 0.13; Slope Fraction $=0.50$ ) 84

Figure A-10 Raw sensible load for the Chicago Medium Office building type. Load response shown as affected by outdoor temperature $(T$, not $\Delta T)$. 85

Figure A-11 Indoor temperature as correlated with temperature differential. Left: no setback. Right: setback enabled. 86

Figure A-12 Chicago Medium Office with no setback. Raw sensible load as affected by envelope temperature differential. Left: sensible load (Intercept $=320.4 \mathrm{MJ} / \mathrm{h}$; Slope $=$ $28.87 \mathrm{MJ} / \mathrm{hC}$ ). Right: sensible ventilation load (Slope $=18.67$; Slope Fraction $=$ $18.67 / 28.87=0.65)$

Figure A-13 Data plots illustrating the patterns and impact of mechanical ventilation. Left: no mechanical ventilation. Right: mechanical ventilation enabled. 88

Figure A-14 Heating and cooling loads in response to changing $\Delta T$. Left: heatingventilation load is removed. Right: raw data (includes heating-ventilation loads). Both plots have mechanical ventilation turned off.

Figure A-15 Raw sensible loads for the Chicago Medium Office building simulated with Phoenix weather as affected by envelope temperature differential. Left: sensible load $($ Intercept $=317.1 \mathrm{MJ} / \mathrm{h}$; Slope $=27.06 \mathrm{MJ} / \mathrm{hC})$. Right: sensible ventilation load $($ Slope = 18.13; Slope Fraction $=18.13 / 27.06=0.67$ ).

Figure A-16 Raw sensible loads for the Chicago Medium Office building simulated with San Francisco weather as affected by envelope temperature differential. Left: sensible load $($ Intercept $=403.9 \mathrm{MJ} / \mathrm{h}$; Slope $=34.05 \mathrm{MJ} / \mathrm{hC})$. Right: sensible ventilation load $($ Slope $=18.84 ;$ Slope Fraction $=18.84 / 34.05=0.55)$.

Figure A-17 Chicago Medium Office building type with Miami Weather. Raw sensible load as affected by envelope temperature differential. Left: sensible load (Intercept $=326.55$ $\mathrm{MJ} / \mathrm{h}$; Slope $=37.66 \mathrm{MJ} / \mathrm{hC}$ ). Right: sensible ventilation load (Slope = 19.38; Slope Fraction $=19.38 / 37.66=0.51)$.

Figure A-18 Phoenix Medium Office building type with Phoenix weather. Raw sensible load as affected by envelope temperature differential. Left: sensible load (Intercept $=$ $327.65 \mathrm{MJ} / \mathrm{h}$; Slope $=29.45 \mathrm{MJ} / \mathrm{hC}$ ). Right: sensible ventilation load (Slope = 18.01; Slope Fraction $=18.01 / 29.45=0.61$ ).

Figure A-19 Raw total load for the Chicago Large Office building type with Chicago weather as affected by envelope temperature differential. Left: total load (Intercept $=$ $4250+/-30 \mathrm{MJ} / \mathrm{h}$; Slope $=410+/-6 \mathrm{MJ} / \mathrm{hC})$. Right: total ventilation load (Slope $=283$ $+/$ - 4; Slope Fraction $=0.69$ ). 
Figure A-20 Sensible load for the Secondary School building type. Left: sensible load $($ Intercept $=779+/-9 \mathrm{MJ} / \mathrm{h}$; Slope $=95+/-2 \mathrm{MJ} / \mathrm{hC} ; \mathrm{I} / \mathrm{S}=8.2 \mathrm{C})$. Right: sensible ventilation load $($ Slope $=12.62+/-0.10 ;$ Slope Fraction $=0.13)$.

Figure A-21 Total load for the Secondary School building type. Left: total load (Intercept = $3256 \mathrm{MJ} / \mathrm{h}$; Slope $=339.1 \mathrm{MJ} / \mathrm{hC} ; \mathrm{I} / \mathrm{S}=9.6 \mathrm{C}$ ). Right: total ventilation load (Slope = 20.04; Slope Fraction $=0.06$ )

Figure A-22 Chicago Small Office building with Chicago weather. Raw sensible load as affected by envelope temperature differential. Left: sensible load (Intercept $=43.4+/-0.3$ $\mathrm{MJ} / \mathrm{h}$; Slope $=3.46+/-0.04 \mathrm{MJ} / \mathrm{hC}$ ). Right: sensible ventilation load (Slope $=1.120+/-$ 0.001 ; Slope Fraction $=0.32$ )

Figure A-23 Chicago Primary School. Left: sensible load $($ Intercept $=$ Intercept $=718+/-5$ $\mathrm{MJ} / \mathrm{h}$; Slope $=63.5+/-1.0 \mathrm{MJ} / \mathrm{hC})$. Right: sensible ventilation load (Slope = $38.8+/-0.3$; Slope Fraction $=0.61)$.

Figure A-24 Warehouse. Left: sensible load (Intercept $=15.5+/-0.5 \mathrm{MJ} / \mathrm{h}$; Slope $=3.68$ $+/-0.16 \mathrm{MJ} / \mathrm{hC}$ ). Right: sensible ventilation load (Slope $=2.35+/-0.02$; Slope Fraction $=0.64)$.

Figure A-25 Warehouse. Left: sensible load (Intercept $=3.8+/-1.5 \mathrm{MJ} / \mathrm{h}$; Slope $=5.3+/-$ $0.4 \mathrm{MJ} / \mathrm{hC})$. Right: sensible ventilation load (Slope $=1.47+/-0.01$; Slope Fraction $=$ $0.28)$.

Figure A-26 Warehouse; hours with zero load are excluded. Left: sensible load (Intercept $=$ $5.7+/-1.7 \mathrm{MJ} / \mathrm{h}$; Slope $=7.1+/-0.5 \mathrm{MJ} / \mathrm{hC})$. Right: sensible ventilation load (Slope = $1.47+/-0.01$; Slope Fraction $=0.21$ )

Figure A-27 Stand-Alone Retail. Left: sensible load (Intercept $=245.4+/-1.5 \mathrm{MJ} / \mathrm{h}$; Slope $=26.5+/-0.3 \mathrm{MJ} / \mathrm{hC})$. Right: sensible ventilation load (Slope = $16.69+/-0.09$; Slope Fraction $=0.63)$.

Figure A-28 Strip Mall Retail. Left: sensible load (Intercept $=187.2+/-1.6 \mathrm{MJ} / \mathrm{h}$; Slope $=$ $35.4+/-0.4 \mathrm{MJ} / \mathrm{hC}$ ). Right: sensible ventilation load (Slope $=14.00+/-0.09$; Slope Fraction $=0.40)$.

Figure A-29 Mid-Rise Apartment. Left: sensible load (Intercept $=128.3+/-0.5 \mathrm{MJ} / \mathrm{h}$; Slope $=12.32+/-0.08 \mathrm{MJ} / \mathrm{hC})$. Right: (none). Slope Fraction $=\mathrm{NA}(0.30$ assumed $)$.

Figure A-30 Sit-Down Restaurant. Left: sensible load (Intercept $=98.0+/-0.5 \mathrm{MJ} / \mathrm{h}$; Slope $=17.65+/-0.14 \mathrm{MJ} / \mathrm{hC})$. Right: sensible ventilation load (Slope $=13.88+/-0.04$; Slope Fraction $=0.79)$.

Figure A-31 Large Hotel. Left: sensible load (Intercept $=1313+/-7 \mathrm{MJ} / \mathrm{h}$; Slope $=149.5$ +/- 1.4 MJ/hC). Right: sensible ventilation load (Slope = 143.3 +/- 1.3; Slope Fraction = $0.96)$.

Figure A-32 Hospital Healthcare. Left: sensible load (Intercept $=1370+/-4 \mathrm{MJ} / \mathrm{h}$; Slope $=$ $55.8+/-0.3 \mathrm{MJ} / \mathrm{hC})$. Right: (none). Slope Fraction = NA (0.8 assumed). ...................... 106

Figure A-33 Outpatient Healthcare. Left: sensible load (Intercept $=704+/-3 \mathrm{MJ} / \mathrm{h}$; Slope $=$ $41.8+/-0.3 \mathrm{MJ} / \mathrm{hC}$ ). Right: sensible ventilation load (Slope $=2.270$ +/- 0.011; Slope Fraction $=0.054)$ 
Figure A-34 Comparison of the calculated total-ventilation load (calculated in post processing using ventilation mass flow) and reported ventilation load (load reported directly from EnergyPlus). The blue line indicates where data would be expected if there was one-to-one agreement. Left: Medium Office. Right: Sit-Down Restaurant. .

Figure A-35 Humidity-ratio differential (outdoor minus indoor) as affected by temperature differential (outdoor minus indoor). Left: raw hourly data. Right: daily averages............... 109 


\section{Tables}

Table 5-1 Candidate Unit Consumption as Affected by Indoor Temperature (locked and

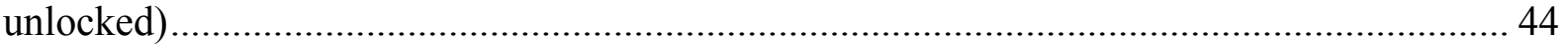

Table 5-2 Capacity levels for each "Number of Stages" setting........................................... 47

Table 5-3 Evaporator Fan Energy (kWhrs) as Affected by Operation Mode........................... 53

Table 5-4 Candidate unit energy consumption as affected by ventilation rate (locked and

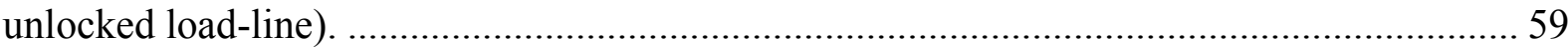

Table 5-5 Candidate Unit Consumption as Affected by Humidity Levels (locked and

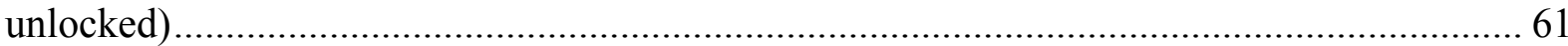

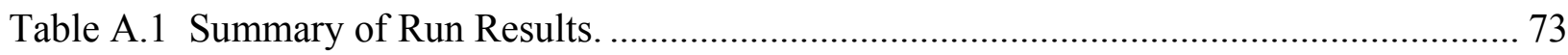

Table A.2 Calculation of S\&I fraction in three cities using the Fast-Food building model....... 79

Table A.3 Calculation of S\&I fraction in three cities using the total-load version of the Fast-

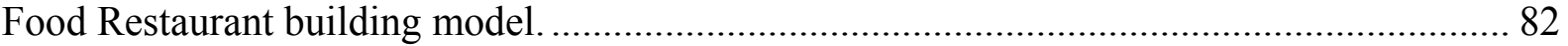

Table A.4 Summary of Chicago Medium Office response model as affected by the weather

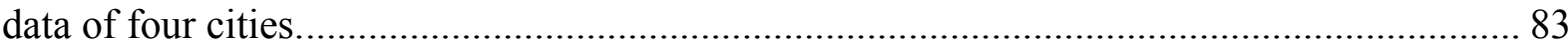

Table A.5 Calculation of S\&I fraction in three cities using a Chicago Medium Office

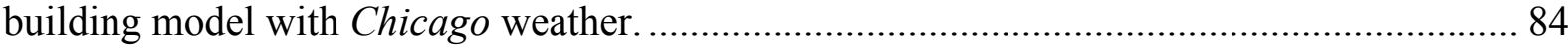

Table A.6 Calculation of S\&I fraction in three cities using the Chicago Medium Office building model with no setback.

Table A.7 Calculation of S\&I fraction in three cities using the linear model resulting from the Chicago Medium Office building with Phoenix weather.............................................. 90

Table A.8 Calculation of S\&I fraction in three cities using the linear model resulting from the Chicago Medium Office building with San Francisco weather.

Table A.9 Calculation of S\&I fraction in three cities using the Chicago Medium Office building model with Miami weather.

Table A.10 Calculation of S\&I fraction in three cities using the Phoenix Medium Office building model with Phoenix weather.

Table A.11 Calculation of S\&I fraction in three cities using the Chicago Large Office building model with Chicago weather. 


\section{INTRODUCTION}

Packaged rooftop air conditioners and heat pump units (RTUs) are used in $46 \%$ (2.1 million) of all commercial buildings, serving over $60 \%$ (39 billion square feet) of the commercial building floor space in the U.S. (EIA 2003). The annual primary (source) energy consumption associated with these units is over 1.3 quads. Therefore, even a small improvement in rated efficiency or part-load operation of these units can lead to significant reductions of energy use and carbon emissions.

The majority of building owners are not familiar with the savings that the new high efficiency RTU products provide.

The Building Technologies Office (BTO) has previously funded development of the RTU comparison calculator (RTUCC). RTUCC is a web-based tool that provides the user a way to compare energy consumption and costs for two units with different efficiencies.

RTUCC calculates the runtime and energy use of evaporator fans, condenser fans, and compressors as affected by weather, mixed-air and outdoor-air conditions, building types, system types, control strategies, and occupancy schedules.

Because RTUCC uses a simplified modeling approach, it completes annual energy performance and economic analyses in approximately 1 second, while the detailed hourly simulation programs such as EnergyPlus and DOE-2 can take several minutes.

The RTUCC's short calculation time is achieved by eliminating the dynamic and sequential hour-by-hour modeling of the commercial building. Building envelope, internal, and ventilation loads are estimated in the RTUCC through the use of a representative linear-response model for each in a set of 14 typical-building types ${ }^{1}$. These building models are scaled to match the capacity of the selected RTU at design conditions for the selected city.

The 8,760 hours in a city's weather year are binned by outdoor temperature. Typically, between 10 and 20 5-degree temperature bins represent the entire year. This compressed representation of the weather is then used to drive the linear-building models and the RTUCC's system simulator.

The simplified building analysis also eliminates the labor needed to specify the details of a building and makes the comparative analysis of RTU units relatively easy.

The end result is a calculation that provides reasonable accuracy for use in comparing the performance of two RTU systems. More detailed predictions of energy use for individual units are best done with hourly building-simulation software.

\footnotetext{
${ }^{1}$ Mid-Rise Apartment, Hospital Healthcare, Outpatient Healthcare, Large Hotel, Small Office, Medium Office, Large Office, Fast-Food Restaurant, Sit-Down Restaurant, Stand-Alone Retail, Strip Mall Retail, Primary School, Secondary School and Warehouse.
} 


\section{Document SCOPE}

This single reference document serves as a user manual. It is essentially an aggregate of the content accessible from the RTUCC website. It contains introductory help material as well as discussions of the engineering methods used in the calculator's engine.

Prior to development work in 2014, the RTUCC could not estimate savings associated with either the RTU Challenge unit or the advanced RTU controls retrofit. Therefore, BTO funded PNNL to enhance the tool so that building owners can compare energy and savings associated with these two new classes of products. These enhancements to the calculator (version 4.3) are described in this document and are generally referred to as the "Specific Candidate Unit" feature. Note that this document is a reflection of the RTUCC web content (html pages and PDFs) as of April 2015 (calculator version 4.3). The RTUCC website and its supporting documentation pages are updated as revisions are made to the calculator. Please refer to the website for updates on documentation. 


\section{QUICK START}

This outline discusses the four basic modes in which the calculator is used to represent a rooftop unit. The intent here is to describe the data requirements for each of these modes and strategies to make the best comparisons with consideration for data availability. This discussion will generally progress from low data-input requirements to high. Help on each of the features below can be accessed by clicking on the question mark next to the feature's name on the calculator's Control page.

\subsection{GENERIC:}

This is the default mode and uses a generic set of DOE-2 coil curves to represent all units.

- No data; a basic demo: A simple click of the submit button will use all the default values of the calculator's features and will generate the basic summary output on the Results page. Turning on the "Show bin calculations" feature will produce a more detailed report. Clicking the "Advanced Features" checkbox displays additional calculator parameters and their default values.

- Basic description of a single-stage RTU: This is the minimum data that is needed for an initial comparison of two single-stage units (with single-speed evaporator fans). This level of comparison can be done without enabling the calculator's "Advanced Features" feature.

- Total capacity (and oversizing factor): The nominal size of the unit in kBtuh. This is the cooling capacity at test conditions established by the Air-Conditioning, Heating, and Refrigeration Institute (AHRI).

- EER: This is the Energy Efficiency Rating (EER) of the unit at AHRI test conditions.

- Costs: Purchase cost (in units of $\mathrm{k} \$: \$ 1,000=1 \mathrm{k} \$$ ) and estimate of annual costs (\$).

- Economizer: This should be checked if the unit can be configured for economizing and it is enabled.

- The environment: This characterizes the environment that essentially determines how hard (and how long) the unit will have to work.

○ Building type: Pick a building type. This establishes a building-load model that predicts cooling load as driven by weather data.

- Location: Pick a state and city to establish the weather data used in driving the building-load model. 
- Schedule: Pick a schedule that best represents the occupancy patterns in the building.

- Setpoint and setback: Pick a setpoint temperature and also a setback temperature to determine control points for occupied and unoccupied periods.

- Economics: This affects the savings and payback calculations.

- Electric Utility Rate: Enter your local electric rate.

- Equipment life: This is the time period over which energy and cost savings are calculated.

- Discounting: If it is difficult to estimate a discount rate, turn this feature off to give non-discounted (simple) payback calculations.

\subsection{GENERIC WITH "ADVANCED FEATURES":}

Clicking the "Advanced Features" checkbox reveals added features that allow the calculator to do more detailed modeling of the single-stage unit. These features also support modeling of the more advanced characteristics of multi-stage and variable-capacity systems. Please refer to the help topics for these features.

- More detailed representation for the single-stage unit:

- Fan and Condenser Power Data: The "Power Inputs" feature allows the user to specify the three power inputs. The evaporator fan power can be estimated as the difference between the gross and net capacity of the unit (expressed in kWatts). The user will notice that the calculator recalculates the EER and condenser power if the evaporator fan or the auxiliary values are edited. A helpful editing pattern is to first edit the "EFn" and "Aux" fields, then re-enter the EER value. Clicking the "Power" button (upper right) will recalculate default values for the power parameters based on the unit's capacity and EER.

- E-Fan and Condenser: Choose between "1-Spd: Always ON" and "1-Spd: Cycles With Compressor." The difference here is that one setting models the evaporator fan as running continuously and the other allows the fan to cycle off with the compressor. Refer to the help topic for this feature for additional information related to unoccupied hours and economizing.

- Humidity: Generally it is best to leave this set to automatic unless there is data on internal humidity levels that might be affected by a separate humidity control system.

- Ventilation: This feature value is automatically calculated based on building type and generally does not need to be edited. 
- Degradation Factor: This is the fractional drop in efficiency of the unit when running at small loads. It will be difficult to get a specific value from a manufacturer. This can be edited to explore the sensitivity of the savings to changes in this parameter. Generally leave this at the default value.

- S/T Ratio: This is the sensible-to-total capacity ratio at AHRI test conditions. This should be available in a manufacturer's performance brochure. Leave at default levels if not available.

- Demand: Generally only use these fields if you must calculate a demand charge. Refer to the help topic for this feature (click on the question mark).

- Staged and Variable-capacity Units: Four of the "Advanced Features" features, facilitate modeling of variable-capacity systems.

○ The features:

- E-Fan and Condenser: Select from three different system types: (1) oneor two-stage units with a single-speed evaporator fan, (2) units with multistage condensers, and (3) units with a variable-capacity condenser.

- Number of Stages: The number of stages in a unit with a staged condenser. Leave this set to 1 for single-stage and variable-capacity units.

- N for Fan Energy Calcs: The value of $n$ used in fan-affinity law calculations for variable-speed fans. This should generally be left at the default value. This feature has no impact for a single-stage unit with a single-speed evaporator fan.

- Condenser Fan: The fraction of condenser power used by the condenser fan at AHRI rating conditions. This fraction can usually be estimated from a manufacturer's brochure. This feature applies only (and can only be edited) if the "V-Spd" unit is selected under the "E-Fan and Condenser" feature.

- Examples:

- Two-stage unit with a single-speed evaporator fan:

Set the "Number of Stages" feature to 2. Set the "E-Fan and Condenser" feature to one of the "1-Spd" choices.

- Two-stage unit with a two-speed evaporator fan: Set the "Number of Stages" feature to 2. Set the "E-Fan and Condenser" feature to one of the "N-Spd" choices.

- Multi-stage unit with a corresponding multi-speed evaporator fan: This is the generalization of the previous case. Change the "Number of Stages" feature to the corresponding level. Set the "E-Fan and Condenser" feature to one of the "N-Spd" choices. 
- A unit with a variable-capacity compressor, variable-speed condenser fan, and variable-speed evaporator fan:

Set the "E-Fan and Condenser" feature to the "V-Spd" choice.

\subsection{Three SPECIFIC UNITS:}

In contrast to the generic correction curves that are used in the modes described above, specific performance curves and algorithms are used to characterize these three units and are written into the computer code of the calculator's computation engine. These proprietary curves and algorithms were provided to PNNL from the manufacturers. There is a Methods page (Section 4.6) that provides background information on these units. Please also refer to the help topic for this feature.

- Specific Candidate Unit: Use this advanced feature to select one of the three units. A selection here automatically sets values for several related calculator features: "E-Fan and Condenser" and "Number of Stages." These automatically-set values may need refinement depending on the specifics of the application. For example, when setting the "Specific Candidate Unit" feature to the "Advanced Controls" value, this type of add-on technology can be applied to either a single-stage or two-stage unit. Similarly, depending on whether the unit's evaporator fan is set to cycle with the compressor or run continuously will determine whether the "E-Fan and Condenser" setting needs refinement.

- Other features: Selecting one these specific units defines its basic nature; however, additional parameters should be set to fully characterize a particular version of the unit. This includes all other RTU-related parameters that are used under the generic-mode approach described above: EER, capacity, power splits, S/T ratio, and degradation factor (if not V-Spd). Use default values if parameters cannot be obtained from a manufacturer's brochure. As done above, the environment and economic parameters need to be set: state and city, schedule, etc.

\subsection{THE SPREADSHEET INTERFACE:}

The spreadsheet provides a general mechanism to characterize full-load and part-load performance of an RTU's condenser using tables from a manufacturer's performance brochure. Please review the help topic for the spreadsheet feature. There is a Methods page that provides additional information (see Section 4.5). The spreadsheet itself has additional instructions and annotation inside. Note that spreadsheet models are not allowed to be used with the "Specific Candidate Unit" feature.

There are three levels of input data. Only the full-load data (first bullet below) is required. The second and third bullets describe optional inputs:

- Full-load total gross capacity and condenser power (required): This data is readily available from manufacturers' performance tables. Some interpolation might be required 
because the spreadsheet needs this data at particular operating temperatures. This data alone can be used to replace the generic coil curves. The good news is that users can build a coil model from this data even if they do not have the additional data described below. If the user prefers to stop at this data level, there are two control cells in the spreadsheet for facilitating putting NAs (Not Available) in the S/T cells (B20 on the FullLoad Performance sheet) and NAs in the part-load tables (B3 on the Part-Load Performance sheet). The NAs will cause the RTUCC to use only the full-load coil data and activate its native apparatus dew point method for S/T modeling and its native partload modeling of fans and condensers for estimating part-load performance.

- Sensible-to-total (S/T) data (not required): The sensible-to-total capacity ratios are also generally available from manufacturers. However, the native $\mathrm{S} / \mathrm{T}$ modeling in the calculator is capable of accounting for changing evaporator flow and condenser capacity levels. For this reason, the spreadsheet's $\mathrm{S} / \mathrm{T}$ model is only recommended for systems with a single-stage and a single-speed evaporator fan.

- Part-load data (not required): This type of tabulated data is currently not available for the public from manufacturers. As a result, this portion of the spreadsheet is only a prototype and demonstrates how this part-load data could be structured in a way related to Integrated Energy Efficiency Ratio (IEER) calculations. The B3 cell on the part-load sheet can be used to turn this off (fill with NAs) or show example values that are generated with consideration for the part-load nature of a single-stage unit or a variablecapacity unit. 


\section{ENGINEERING MethodS}

This series of pages describes the engineering methods behind the rooftop unit comparison calculator (RTUCC). Each section starts with a discussion and concludes with a short outline that emphasizes key points.

- Establishing Loads

- Sensible Load-lines

- An Example: schedule and load data

- Corrections to Tested Performance

- Total Capacity and Condenser Power

- Sensible Capacity

- Manufacturers' Data and the Spreadsheet Interface

- Three Specific Units

- $\quad$ Equipment Response to Loads

- Mixed Air and Coil Conditions

- Capacity Corrections

- Load Balance

- Condenser Power

- Evaporator-fan Power

- Latent Loads

- Economics

- Equations

- Charting Life-Cycle Costs

\subsection{ESTABLISHING LOADS: SENSIBLE LOAD-LINES}

The RTUCC uses weather data to conduct a binned energy analysis of the rooftop unit for cities across the United States. Weather tape data (outdoor dry-bulb and coincident web-bulb) was binned in 5 degree increments and filtered by the selected occupancy schedule. The result is a database of hours (in each bin) and coincident wet-bulb temperatures for each city and occupancy schedule combination.

The first step in the binned analysis is to determine the air-conditioning load for each weather bin. Because building characteristics are not explicitly entered for the RTUCC, building loads must be inferred by assuming the unit would be installed in a building suitable for its specified capacity. Load behaviors for this hypothetical building are established via a "Total" sensible load-line and the "Non-ventilation" sensible load-line. These load-lines are defined by the yellow line markers in Figure 4-1. 


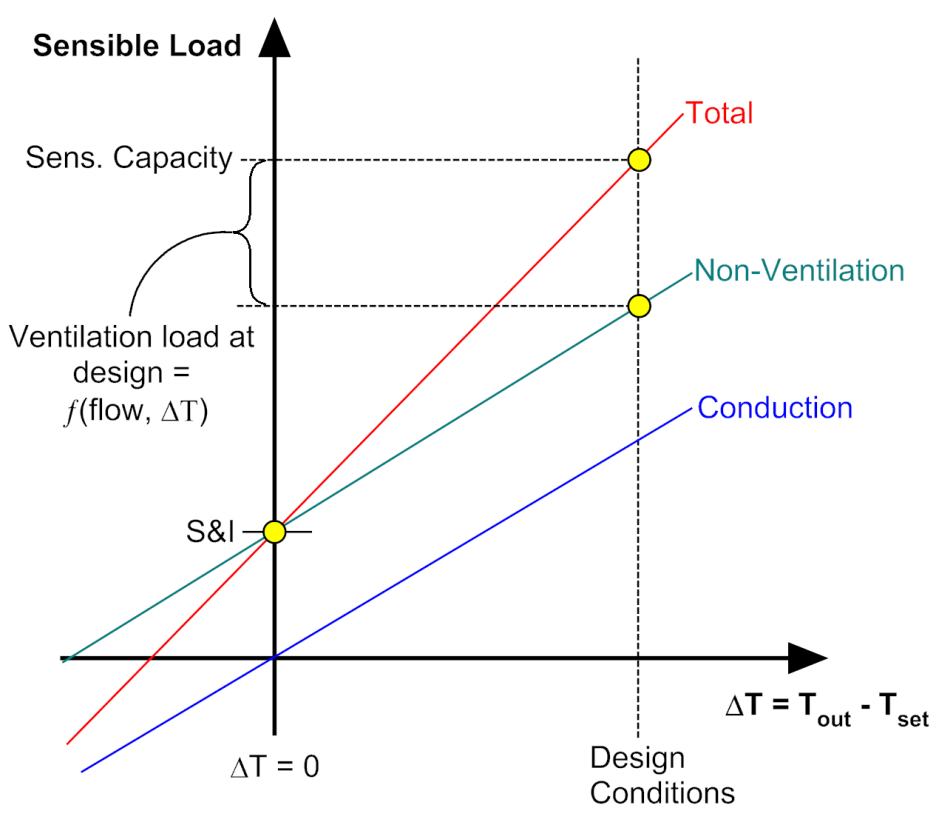

Where S\&I are the SOLAR and INTERNAL gains

Figure 4-1 Load-Line Concept Drawing

Fundamental to the cost estimator is the assumption that total sensible loads and sensible capacity are balanced at design conditions. This balance is achieved by scaling a linear buildingload model $^{2}$ until the load equals the unit's capacity at design conditions.

Another principal concept is that internal gains and solar loads contribute to the total sensible load. This essentially produces an intercept in the load model such that there is a cooling load even when the outdoor temperature matches the setpoint. This two-point nature to the load model allows for quantitative adjustments to better reflect various building types. For example, the "Building Type" feature allows the user to select a building type that is internally dominated and insensitive to weather (high internal gains as with a multi-story office building) or one more coupled to the weather (low internal gains as with a warehouse). The EnergyPlus load models for each building type can be manually edited to produce custom versions of each building-load model. The analysis behind the load models can be reviewed in this document (Appendix A). There is also related discussion in the help for the "Building Type" feature.

The RTUCC uses a sensible load analysis formulation. Humidity impacts on performance are accounted for as they affect the unit's sensible capacity to meet the total sensible load (and also the effects on system power draw). Interior humidity conditions can be set to automatically track outside conditions (by assuming inside humidity ratio is equal to outside humidity ratio), or they can be set to a constant (relative humidity) value. Note that energy usage associated with other humidity controlling devices is not included in the estimates by the calculator.

Key points in this section:

\footnotetext{
${ }^{2}$ Load models are also referred to as "response models" (see Appendix A).
} 
- Establish high point of the total sensible load-line

○ Total sensible load=Total sensible capacity @ design conditions

- Use rated sensible capacity corrected to design conditions

- Establish two points for the non-ventilation line

- Subtract sensible ventilation load at design conditions

- $\mathbf{S V L}=f\left(\right.$ ventilation mass flow, $\left.\mathrm{t}_{\text {out }}-\mathrm{t}_{\text {in }}\right)$

- Solar and internal gains point (the intercept)

- Value is calculated based on user's selection of building type and location.

Calculation is based on EnergyPlus load models for building types used by the American Society of Heating, Refrigerating and Air-Conditioning Engineers (ASHRAE).

\subsection{ESTABLISHING LOADS: AN EXAMPLE}

Fan capacity, not used in ventilation, can optionally be used in economizing the load. The economizer reduces the sensible load, at outdoor temperatures below the setpoint, by fully opening the outside-air damper and bringing in additional ventilation air. When economizing, the supply fan runs at full speed.

In the example chart produced for Wichita by the RTUCC (Figure 4-2), the "After Economizer" trace shows the final load data that will drive the runtime calculation (this trace is reduced to zero for those bins where the economizer can satisfy all the cooling load).

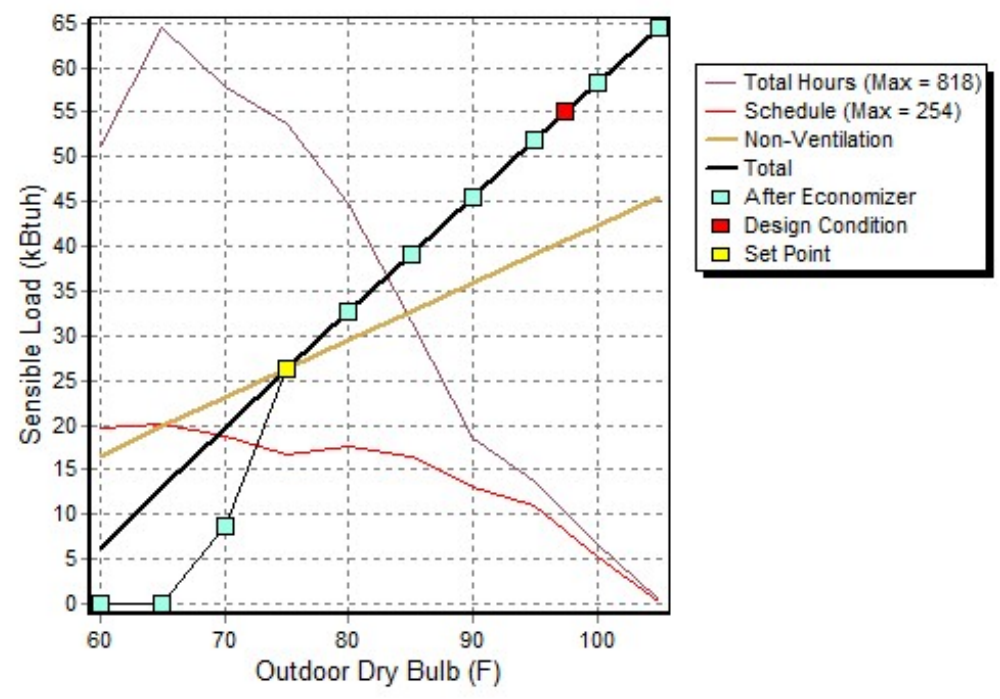

Figure 4-2 Loads and Hours: Wichita, KS; Medium Office

For temperature bins where the economizer cannot satisfy the load on its own, supplemental direct expansion (DX) cooling is modeled in the calculator in what is called integrated mode (see the $70^{\circ} \mathrm{F}$ bin in Wichita chart). In this mode, the unit balances the load against the two cooling sources to determine how much DX runtime is needed in the hour. During the economizer-only portion of the hour, the fan runs at full speed. During the DX-only portion of the hour, the fan 
runs at full-speed for a single-speed system. However, for a variable-capacity unit, the DX-only portion of the hour runs at a fan speed that corresponds to the first-stage of a staged unit or the minimum capacity of a unit with a variable-capacity condenser.

The final step in presenting the load data to the unit is to determine the number of hours in which the unit experiences that load. In the example chart for Wichita, with a schedule of Monday Friday, 7am - 7pm, the hour distribution, as binned by outside temperature, is shown normalized, with a fine red line ( $\max$ value $=254$ hours). This line is labeled "Schedule." The potential total hours, as would be the case if no schedule were filtering the hours, is shown by the fine darkbrown line, labeled "Total Hours." This total-hours trace is displayed for use as a reference to give the user a sense of the impact of the filtering by the schedule. When an "All Day, All Week" schedule is selected, these two traces (filtered and unfiltered) are identical and lay on top of each other.

When considering how unit performance varies from city to city, it is useful to keep in mind that the building-load model (in this case: "Office-Medium") is scaled to match the capacity of the candidate unit at design conditions. As a result of this explicit balance, design temperature is not by itself a primary driver of energy usage, but rather two associated weather characteristics have more impact:

1. Hour count in the cooling bins (longer cooling season means more energy use).

2. The skew of the hour distribution (the more skewed toward high temperatures, the more time at high load and low capacity; the result is longer runtime per bin hour).

The RTUCC reports calculation results for any temperature bin where there is a positive cooling load (before economizer reductions).

Key points in this section:

- Economizer

- Sensible load is reduced by fully opening the outside-air damper and running the supply fan at full speed.

- Hours of operation from weather tapes

- Schedule acts as filter

- Binned by dry-bulb (with coincident wet-bulb)

\subsection{Corrections to Tested Performance: CaPacity and Condenser Power}

Because of the binned analysis in the calculator, there is need to determine equipment performance at ambient conditions other than the AHRI rating conditions $\left(\mathrm{ODB}=95^{\circ} \mathrm{F}\right.$, $\mathrm{EWB}=67^{\circ} \mathrm{F}$ ). The DOE-2 cooling correction curves shown in Figure 4-3 allow performance to be estimated at other environmental conditions. These corrections are functions of outdoor dry-bulb temperature (ODB) and entering (mixed air) wet-bulb temperature (EWB). 


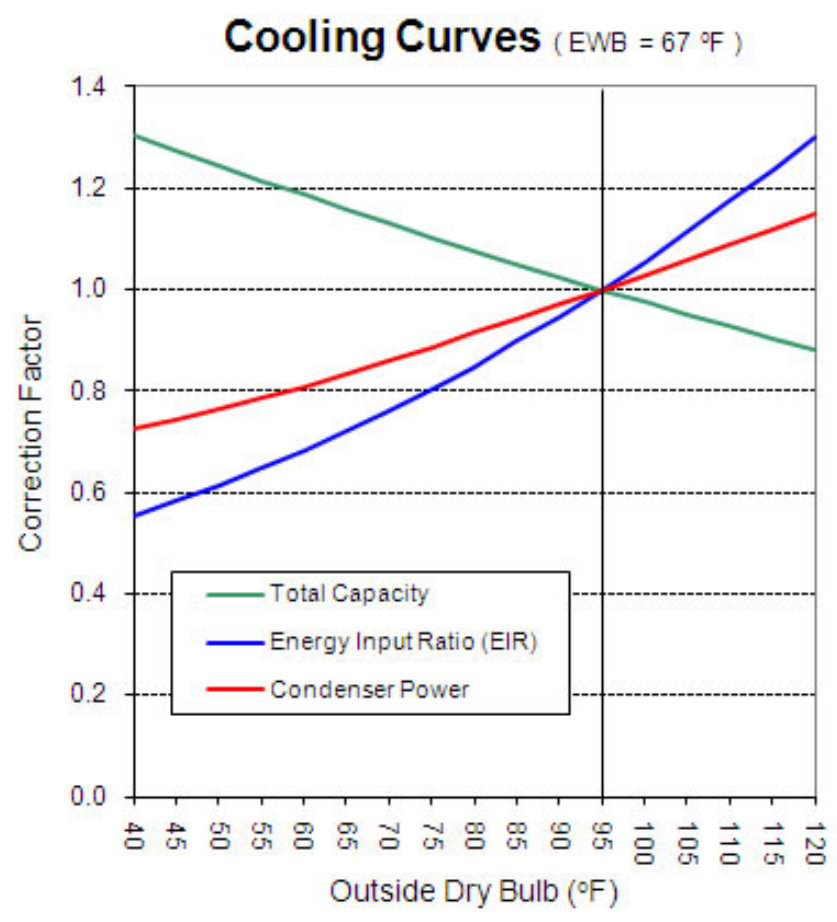

Figure 4-3 Illustration of DOE-2 Correction Curves (entering wet-bulb temperature $=67^{\circ} \mathrm{F}$ )

In making corrections to system power draw, the calculator makes use of two of these DOE-2 correction functions. In DOE-2 reference literature, these can be identified in the commercial package DX group of functions:

COOL-CAP-FT: correction to the rated total gross cooling capacity

COOL-EIR-FT: correction to cooling energy input ratio $\left(\mathrm{EIR}=\mathrm{Btu}_{\mathrm{in}} / \mathrm{Btu}_{\mathrm{out}}\right)$

The calculator's correction function for condenser power draw is derived as the product of these two functions.

The system performance calculation page serves to demonstrate the correction methods described in this section and the next. The results of corrections to total gross capacity are seen in columns with header names "TCap (Gross)."

Key points in this section:

- DOE-2 Correction Curves:

- Total Gross Capacity $=\mathrm{TGC}_{\text {rated }} * f_{\mathrm{TC}}(\mathrm{ODB}, \mathrm{EWB})$

- Condenser Power $=\mathrm{CP}_{\text {rated }} * f_{\mathrm{TC}}(\mathrm{ODB}, \mathrm{EWB}) * f_{\mathrm{EIR}}(\mathrm{ODB}, \mathrm{EWB})$ 


\subsection{Corrections to Tested Performance: Sensible Capacity}

In making predictions of sensible capacity at conditions differing from AHRI test conditions, the calculation engine makes use of the iterative apparatus dew point and bypass factor method. This method answers the question: At what $\mathrm{S} / \mathrm{T}$ ratio does the cooling process preserve the bypass factor that is determined at AHRI rating conditions?

This method acts to first characterize the cooling coil with a bypass factor based on its sensible and total capacity at AHRI test conditions (see left graph in Figure 4-4). This bypass factor can then be used to predict the sensible-to-total ratio of the coil at entering conditions, flow rates, and total capacity levels, other than those at AHRI test conditions (see right graph in Figure 4-4). The relative effect of these corrections on sensible and total capacity is illustrated in Figure 4-5.
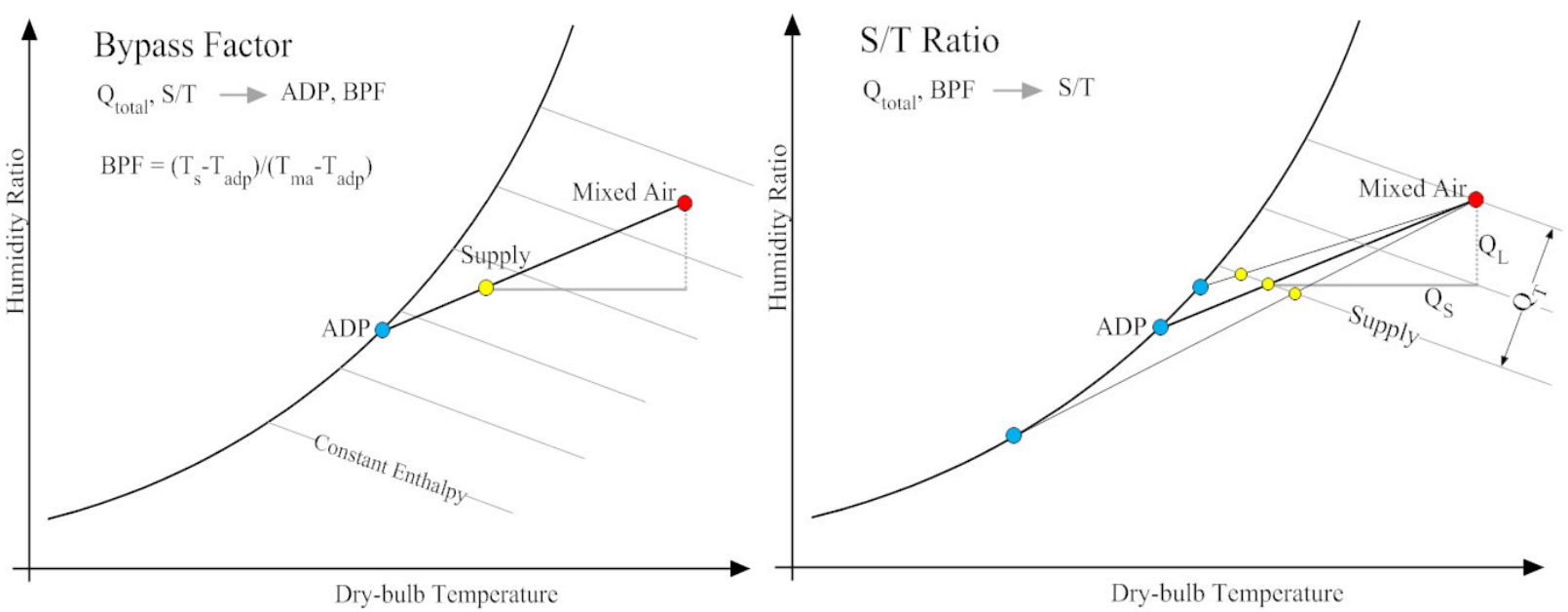

Figure 4-4 Left: Graphical illustration of the definition of the bypass factor: ratio of segment yellow-blue to segment red-blue. Right: Graphical illustration of the iterative technique used to determine the sensible-to-total ratio from a given bypass factor and total capacity.

There is a separate system performance calculation page that serves to dynamically illustrate the Apparatus Dew Point and Bypass Factor (ADP/BPF) method described here. The results shown in the "S/T" columns are the result of the iterative calculation using the ADP/BPF method. Of the three variations of this $\mathrm{S} / \mathrm{T}$ result, the $\mathrm{S} / \mathrm{T}$ (BP-EP) is the one actually used in the calculation engine. This version has algorithmic similarities with the form of the method that is used in the EnergyPlus system simulator (EnergyPlus Engineering Reference, 2014).

The letters "S" stands for supply, "E" for entering, and "O" for outside, "HR" for humidity ratio, "DB" for dry-bulb, and "WB" for wet-bulb. The "BPF" factor, shown at the top row of the second table, is the calculated bypass factor at the user-entered AHRI conditions. All columns to the right of and including the "BPF" column are calculated with the ADP/BPF method.

To run the calculation, enter system performance data (at AHRI test conditions) in the text boxes in the top row and then press the "Submit" button. This produces a table of predicted coil performance for a variety of entering conditions. The second row of inputs allows you to change 
the capacity level and air flow rate that the projection table is based on (by default, these values are left equal to the AHRI conditions specified in the first row of inputs).

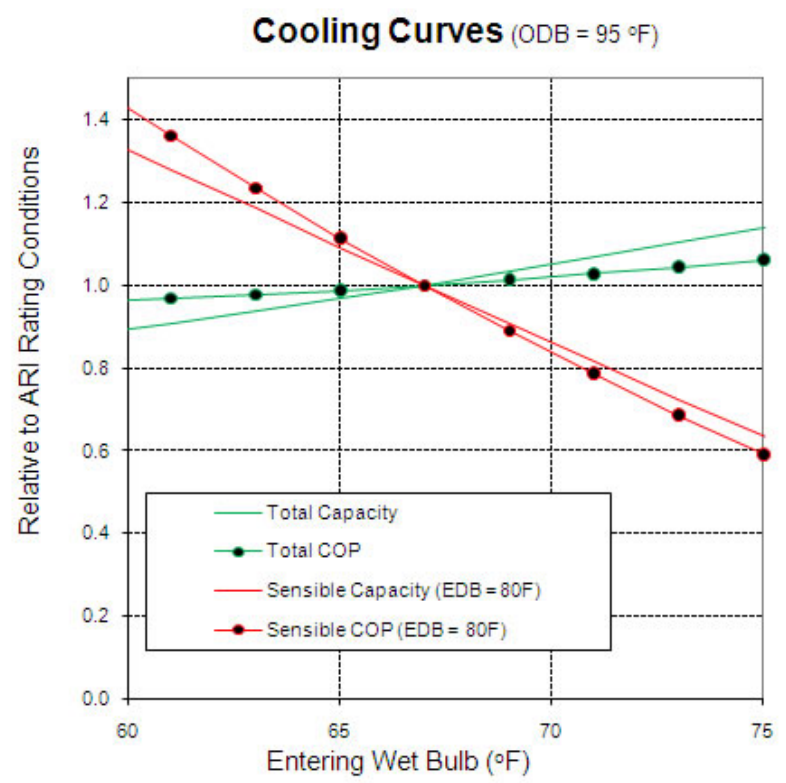

Figure 4-5 Total and sensible capacity data normalized by corresponding values at AHRI rating conditions. This graph shows the stronger dependence of the sensible capacity on the humidity of the entering (mixed) air.

Key points in this section:

- Sensible / Total

- The sensible/total capacity ratio is determined by an iterative technique based on the apparatus dew point and bypass factor method (ADP/BPF). This method is dependent on the $S / T$ ratio (at rating conditions), air flow rate, total capacity, $O D B, E W B$, and $E D B$.

- This iterative technique can be represented functionally: $\boldsymbol{S} / \boldsymbol{T}_{\mathrm{ADP}}=\boldsymbol{S} / \boldsymbol{T}_{\mathrm{ADP}}\left(S / T_{\text {rated, }}\right.$ Flow, Capacity, $\left.O D B, E W B, E D B\right)$

- Sensible capacity is the product of this ratio and the total capacity.

○ $\boldsymbol{S C}=\boldsymbol{S} / \boldsymbol{T}_{\mathrm{ADP}} * \boldsymbol{T C}(O D B, E W B)$

\subsection{Corrections to TeSted Performance: THE SPREAdSheEt INTERFACE}

For manufacturers' performance data specified in detail via the spreadsheet interface, the regression forms outlined above are used to represent the unit in the calculation engine. This detailed data (except for degradation data), is commonly provided in a manufacturer's detailed specification brochure. These unit-specific regression models serve to replace the default DOE-2 regression models for capacity and power draw. The $\mathrm{S} / \mathrm{T}$ regression model replaces the default apparatus dew point bypass-factor method for predicting sensible capacity. The degradation 
regression model replaces the default linear degradation model that is characterized by the "degradation factor" on the Controls page.

Regressions are done for four categories of performance data: (1) gross capacity, (2) condenser power, (3) sensible-to-total capacity ratio, and (4) part-load degradation. The polynomial regression forms (see outline below) illustrate the dependence on outdoor dry-bulb (ODB), entering wet-bulb (EWB), entering dry-bulb (EDB), and load fraction (LF). Net performance relationships are determined by including the effects of the evaporator fan. Note that the term entering refers to the mixed air entering the evaporator coil. Examples of these regression-base correction models are shown in Figure 4-6 and Figure 4-7.

The part-load degradation data that is needed for the regression above is a 16-point table of EER data, indexed by load fraction and outdoor temperature. This is basically an expanded version of the EER dataset that is required for an IEER calculation (4-points). This EER table differs from that used in the IEER calculation in that the evaporator fan power has been removed from the EER calculations. The first step in processing this table is to normalize all the EER data by the corresponding full-load values. This results in a 16-point normalized representation of the partload efficiency factor (the values in the full-load row are all 1.00 after normalization).

The graphs to the right are examples of these regression models for one particular unit. Regression lines are in blue and corresponding raw data is marked by red circles.

These specific regression models are utilized in ways similar to the default methods outlined above and in the previous pages. Equipment performance at operating conditions is calculated by applying these regression models to shape (or correct) the AHRI rated performance specifications.

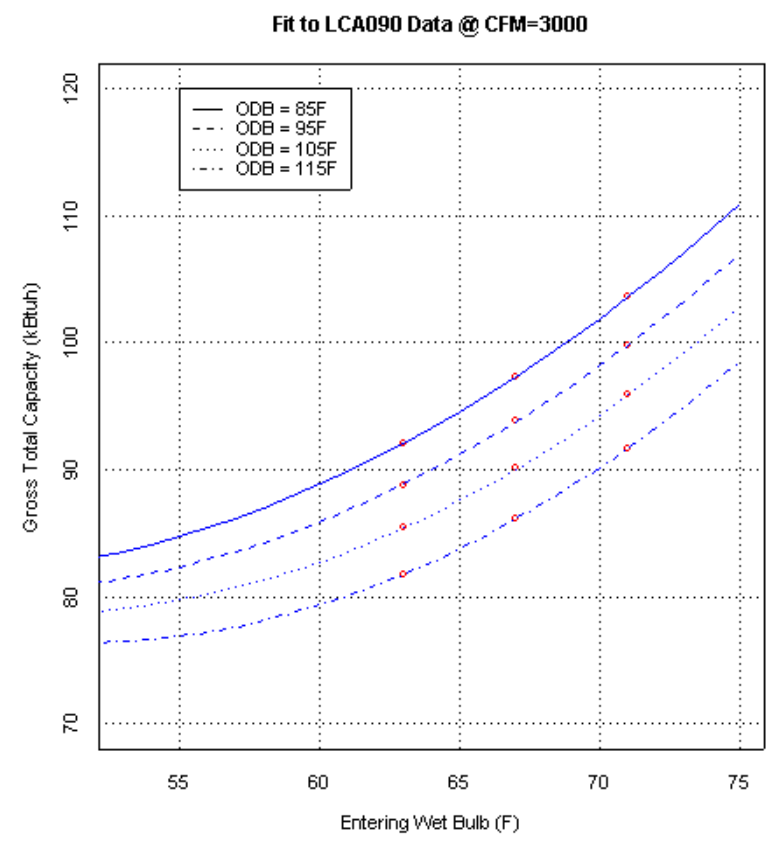

Figure 4-6 Example of performance-correction relationships for total capacity. 

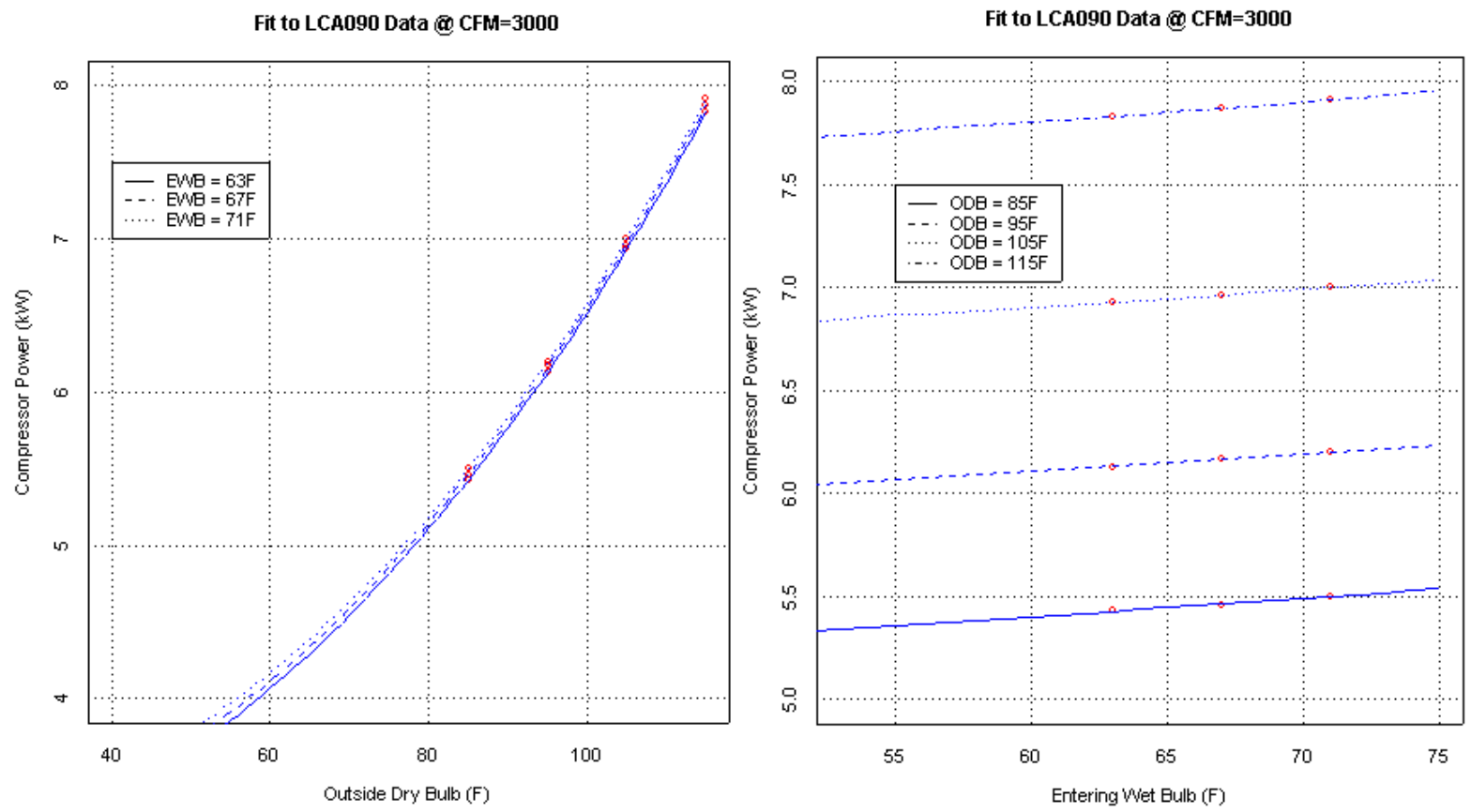

Figure 4-7 Examples of performance-correction relationships for power.

This outline summarizes the model forms used in the regressions and the functional forms developed from them:

- Regression of manufacturers' performance data:

- $f_{\mathrm{GTC}}=$ Gross Total Capacity Correction $=$

Regression_GTC( ODB, EWB)/ Regression_GTC( 95,67$)$

Polynomial model form $=\mathrm{ODB}+\mathrm{ODB}^{\wedge} 2+\overline{\mathrm{EWB}}^{\wedge} 2+\mathrm{ODB}^{*} \mathrm{EWB}+$

$\mathrm{ODB}^{\wedge} 2 * \mathrm{EWB}^{\wedge} 2$

- $f_{\mathrm{CP}}=$ Condenser Power Correction $=$

Regression_CP(ODB, EWB) / Regression_CP( 95, 67)

Polynomial model form $=\mathrm{ODB}^{\wedge} 2+\mathrm{EWB}^{\wedge} 2+\mathrm{ODB}^{*} \mathrm{EWB}+\mathrm{ODB}^{\wedge} 2 * \mathrm{EWB}^{\wedge} 2+$ $\mathrm{ODB}^{\wedge} 3 * \mathrm{EWB}^{\wedge} 3$

- $f_{\mathrm{S} / \mathrm{T}}=$ Sensible to Total Ratio $=$

Regression_S/T( ODB, EWB, EDB)

Polynomial model form $=\mathrm{EWB}+\mathrm{EDB}+\mathrm{EWB} * \mathrm{EDB}+\mathrm{EWB}^{\wedge} 2 * \mathrm{EDB}+$

$\mathrm{ODB}^{*} \mathrm{EWB}^{*} \mathrm{EDB}^{\wedge} 2+\mathrm{ODB}^{*} \mathrm{EWB}^{\wedge} 2 * \mathrm{EDB}$

- $f_{\text {PLDF }}=$ Part-Load Degradation Factor $=$

Regression_DegradationFactor( Load Fraction, ODB)

Polynomial model form $=$ Intercept $+\mathrm{LF}+\mathrm{LF}^{\wedge} 2+\mathrm{LF}^{*} \mathrm{ODB}+\mathrm{LF}^{\wedge} 2 * \mathrm{ODB}$ 
- Apply corrections to gross AHRI values, then add the effects of fans to give net performance relationships

- Net Total Capacity $=f_{\text {GTC }}(\mathrm{ODB}, \mathrm{EWB}) * \mathrm{GTC}_{\mathrm{AHRI}}-\left(\mathrm{P}_{\text {evap-fan }} * 3.413\right)$

- Net Sensible Capacity $=f_{\mathrm{GTC}}(\mathrm{ODB}, \mathrm{EWB}) * \mathrm{GTC}_{\mathrm{AHRI}} * f_{\mathrm{S} / \mathrm{T}}(\mathrm{ODB}, \mathrm{EWB}, \mathrm{EDB})$ $\left(\mathrm{P}_{\text {evap-fan }} * 3.413\right)$

- Condenser Power $=f_{\mathrm{CP}}(\mathrm{ODB}, \mathrm{EWB}) * \mathrm{CP}_{\mathrm{AHRI}}$

- $\mathbf{E E R}=\mathbf{N T C} /\left(\mathbf{C P}+\mathrm{P}_{\text {evap-fan }}+\mathrm{P}_{\text {aux }}\right)$

\subsection{Corrections to Tested Performance: Three SPECIFIC RTUS}

The RTUCC was adapted in version 4.3 to support the evaluation of three specific highperformance RTU units. The performance curves and algorithms that characterize these units are explicitly written into the computer code of the calculator's computation engine. This contrasts with the spreadsheet interface (as described in previous Methods sections) that facilitates a general interface for characterizing full-load and part-load performance.

The following outline briefly explains each of the three computational approaches used in representing these units.

- Advanced Controls: This is a retrofit package and includes adding a controller and a variable-frequency drive to the supply fan. The controller also adds an integrated economizer option. Although some controllers in the market are capable of adding demand-controlled ventilation, the RTUCC does not yet provide that feature. The following outline lists the fan levels set by the Advanced Control system. For two-stage RTUs, the first-stage cooling runs the fan at $75 \%$ and second-stage cooling runs the fan at $90 \%$. Single-stage RTUs address all calls for cooling by running the fan at $90 \%$.

- No call for cooling:

- Fan runs at $40 \%$

- Normal operation

- First-stage call:

- ODB $>=70 \mathrm{~F} ;$ Fan at $75 \%$

- ODB < 70F; Fan at $90 \%$

- Second-stage call: Fan at $90 \%$

- Economizer

- Fan at $75 \%$

- Fan at $90 \%$ (integrated)

- Three-Stage RTU: This is a high-performance unit with three stages. Stage capacities are approximately 40,60 , and $100 \%$ of full-load capacity. This unit uses a three-speed evaporator fan and single-speed condenser fans.

This three-stage unit is represented by six correction curves. Each of the three stage levels is modeled by a pair of correction curves: (a) one for modifying the rated gross capacity, and (b) one for modifying the rated energy input ratio (EIR). Each of these six curves has a 
polynomial form and is a function of the wet-bulb temperature entering the evaporator coil and the outdoor dry-bulb temperature entering the condenser coil.

- $\quad$ RTU with Variable-Speed Compressor: This is a high-performance unit that uses a combination of staging and variable-capacity control and a variable-speed evaporator and condenser fans. This unit first engages its variable-capacity condenser to satisfy smaller loads; at higher loads, the additional stages are also used.

- The variable-speed compressor unit's performance curves (polynomial form) have been modified to estimate full-load capacity. This modified curve was generated from test data that corresponds to full-load operation. This modified curve is used to determine full-load capacity values at bin conditions. Full-load capacities and corresponding building loads are used to estimate the load fraction (sensible coil load/sensible coil capacity). Sensible to total capacity ratios are determined with the ADP/BPF (apparatus dew point/bypass factor) method.

- Load balance equations are solved by iteratively searching for the $f f$ value (flow) at which the corrected capacity balances the load. A fan-flow based modification function is used in the capacity correction. During the iterative process, sensible capacity is determined with the ADP/BPF method.

- This ff value (determined in the load balance) is then used to modify the EIR and capacity corrections to determine the appropriate power consumption of the condenser.

- These correction curves fully capture the hybrid nature of the condenser unit. They represent the two-stage (one variable-capacity stage and one fixed-capacity stage) design and the performance of the condenser fan. There is no need for explicit modeling of the staging or the condenser fan used in this system.

- The evaporator fan performance is estimated with a power-law model (fan-affinity law) using the default exponent of 2.5. The model depends on the $f f$ value as described above.

- The evaporator fan runs at $40 \%(f f=0.40)$ during times of pure ventilation.

- If the coil load is less than the minimum capacity of the RTU (15\%), the condenser runs less than the full hour. In this case, the runtime equals the ratio of the coil load to the minimum capacity. In all other cases, the variable-capacity condenser runs the full hour.

Selection of either the Three Stages or the Variable-Speed Compressor RTU options invokes corresponding sets of capacity and efficiency correction curves for that unit. The "Advanced Controls" option only affects the behavior of the evaporator fan and therefore uses the default DOE-2 corrections curves.

\section{Discussion:}

The RTUCC can model generic versions of the variable-speed and three-stage units described above. This is done by setting the "Specific Candidate Unit" feature to "None" and setting the "E-Fan and Condenser" feature to either "V-Spd: Always On" or one of the "N-Spd..." options.

- Variable-Speed Compressor: The general iterative approach used in the load balance described above is also used to model the generic variable-capacity unit. The iteration 
searches for the capacity fraction at which the unit's sensible capacity balances the sensible load. The key differences here are: (1) that the part-load capacity and part-load compressor power at bin conditions are determined by scaling the full-load value by the capacity fraction (no specific modifying curve), and (2) the default DOE-2 curves are used to represent corrections to capacity and EIR as affected by operation conditions.

- Three-Stages: The generic model uses the DOE-2 correction curves for each stage level.

Please refer to the Quick-Start (Section 3) for more information on generic modeling.

\subsection{EQUIPMENT RESPONSE To LOADS}

The plot of equipment performance in Figure 4-8 is generated when the "Show bin calculations" option is selected. The plot data is from five of the columns in the bin-calculations tables.

- The red (TCF) line is the correction to the total capacity.

- The green (PCF) line is the correction to the system power draw.

- The brown line is the $\mathrm{S} / \mathrm{T}$ ratio.

- The lines with square markers indicate system energy usage and are normalized to allow plotting with the correction factors.

- Blue square markers indicate consumption from the condenser.

- Red square markers indicate consumption by the evaporator fan.

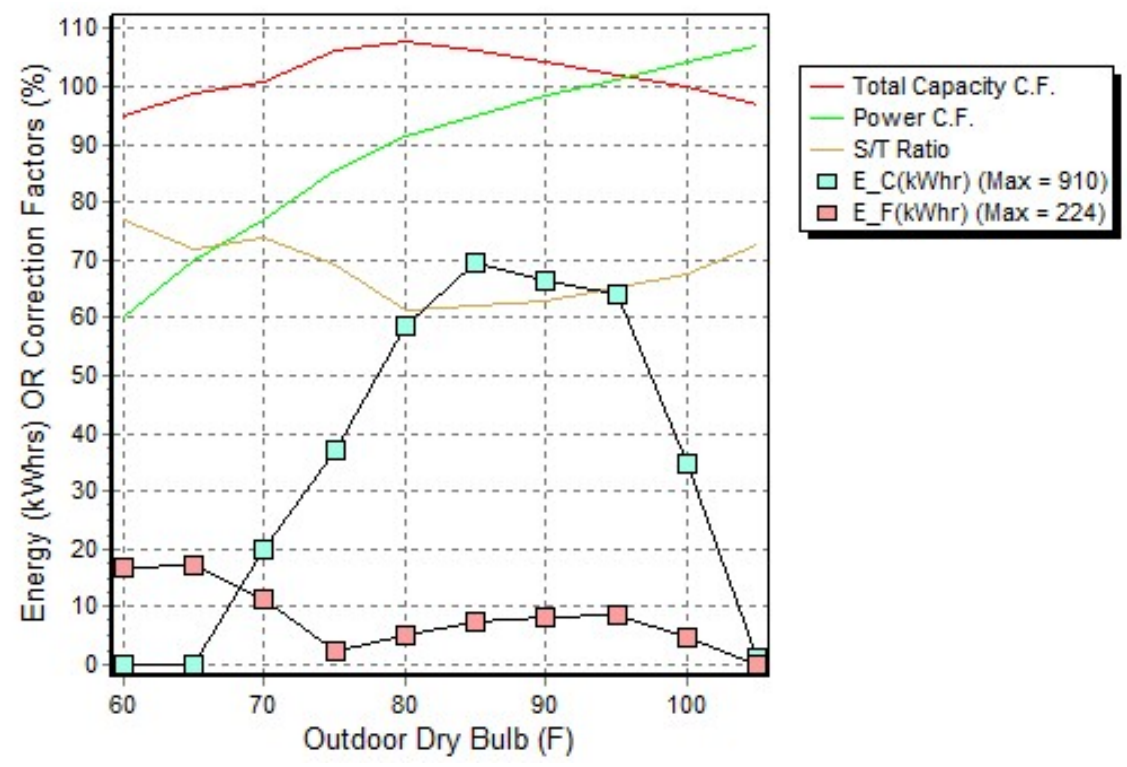

Figure 4-8 Wichita, KS: Equipment Performance

Modeling the unit's response to the cooling load starts with corrections to the total gross (fullload) capacity as affected by outdoor and mixed-air conditions and evaporator fan air-flow rate. This is followed by solving for the condenser runtime or doing an iterative search for the 
condenser capacity level at which the RTU's sensible capacity balances the sensible cooling load. Once the runtimes (or capacity levels of staged or variable capacity units) are determined, fan energy and condenser power levels are established and corresponding energy usage is calculated.

This outline provides a high-level description of this sequence of steps:

- Entering (coil) conditions are determined with a mixed-air calculation based on ventilation rate and economizer usage.

- Capacity Corrections:

- Total capacity is calculated as corrections to gross AHRI rated capacities as affected by environmental conditions (outdoor dry-bulb, and entering wet-bulb and dry-bulb). Corrections can be in one of three forms: (1) generic DOE-2 corrections curves, (2) spreadsheet-based correction curve, and (3) specific manufacturers' corrections as selected by the "Specific Candidate Unit" feature.

- Sensible capacity is calculated using the apparatus dew point bypass factor method. Outside temperature, mixed-air temperature and wet-bulb affect the estimate of sensible capacity. For staged and variable-capacity systems, capacity level and fanspeed level also affect these calculations. (Note that the spreadsheet interface supports regression modeling of the sensible-to-total ratio data as provided in manufacturers' data. However, capacity level and fan level effects on $\mathrm{S} / \mathrm{T}$ are not considered if $\mathrm{S} / \mathrm{T}$ data is supplied with the spreadsheet.)

- Load balance between sensible capacity and sensible load can be calculated for three types of RTU:

- Single-stage system: The unit runs for part of the hour (cycles).

- Multiple-stage systems: As a multi-stage unit attempts to match its capacity to the cooling load, it will progress from lower to higher stage capacities. This search for balance starts with its first stage, then steps through pairs of intermediate stages, and potentially ends at its highest stage level. During times of intermediate load levels, the lower stage level (of the stage pair) and the associated compressors that comprise it, run the whole hour. The difference between the two levels in the stage pair (a single compressor) runs part of the hour (cycles) and its performance is degraded according to runtime fraction.

- Variable-capacity compressors: Through an iterative calculation, the unit's compressor capacity and fan flow are adjusted to balance the sensible capacity with the sensible load.

- Condenser-power corrections:

- Full-load corrections are made to the rated efficiency as affected by environmental conditions. In a process that mirrors the correction methods for capacity, full-load condenser power corrections are made based on the efficiency and capacity corrections.

- Part-load corrections:

- For systems that cycle, degradation is calculated using a linear relationship depending on the user specified "Degradation Factor."

- For systems with variable-capacity condensers, as the system unloads, the compressor power scales down in proportion to the capacity, and the 
- Evaporator fan power:

condenser fan power follows fan-affinity laws. The variable-speed system does not need to cycle and therefore has no part-load degradation.

- (Note that the spreadsheet interface provides a polynomial-regression model of condenser power as a function of load fraction and outdoor dry-bulb. This can be used to model fixed or variable capacity systems.)

- Full-load evaporator fan power can be specified on the Controls page or the default value can be used as determined by the unit's rated total capacity. The following linear relationship is based on a survey of RTUs: Power_FullLoad $=(0.0132 *$ TotalCapacity_kBtuh) -0.2283 .

- Part-load corrections:

- Single-speed fans run at the same speed (and power) whenever they are on.

- For multi-speed fans, affinity laws are used to estimate fan-power draw at part-load conditions. The fan-flow rate scales with capacity as the unit unloads.

Power_PartLoad $=$ Power_FullLoad $*((\text { CFM_PartLoad }) /(\text { CFM_FullLoad }))^{n}$, where $n$ has a default value of 2.5 but can be specified on the Controls page.

The following outline discusses key algorithmic elements of the calculation engine:

- The RTUCC accounts for fan savings of evaporator and condenser fans that change speeds as capacities change. For systems with a variable-capacity compressor, both the evaporator and condenser fans will operate at reduced power levels. For staged systems, only the evaporator fan reduces speed; the condenser fans turn on/off as their corresponding compressor turns on/off. Reduced fan speeds correspond to reduced fan power. For example, a fan running at half speed will operate at $1 / 8$ th power. This is predicted by the fan-affinity laws when a value of 3 (for $n$ ) is used in the model (e.g. $\left.(1 / 2)^{3}=1 / 8\right)$. The $n$ value can be changed by the user on the RTUCC Controls page. For systems with condenser fans that change speed, the fraction of the condenser power at test conditions must be known (or estimated) to facilitate the calculation.

- With RTUCC version 4.3 comes improved modeling of the sensible capacity of the unit as affected by flow changes. This means the calculator is now representing row-split units (evaporators in series) and therefore a single-speed fan on a two-stage unit will yield a much more sensible first stage (this makes it more efficient in terms of satisfying the sensible load as governed by a sensible thermostat). These flow effects are also visible for higher multi-stage and variable-speed compressor systems. They become more sensible as they run at lower condenser capacities (and fan flows).

- The "Advanced Controls" retrofit (an option available under the "Specific Candidate Unit" feature) acts to reduce the speed of a single-speed evaporator fan. When this option is selected, fan-related corrections to gross-total capacity and efficiency are made based on the fan-speed fraction (relative to rating conditions). These are the standard flow-driven correction curves that are part of the DOE-2 set of curves. These corrections have a relatively small impact on savings calculations for the Advance Control retrofit with less than a $1 \%$ reduction in overall savings.

- Modeling of the Advanced Controls retrofit unit in the RTUCC indicates a small condenser penalty (negative condenser energy savings). This is because the reduced fan 
speeds make the unit less sensible (more latent because the coil is colder), so there is more condenser runtime needed to satisfy the sensible load. There is some supporting evidence of this in the Pacific Northwest National Laboratory (PNNL) field assessment data (Wang et al. 2013). This is indicated in the field data by overall unit savings that are less than the fan savings. However, it is important to note that the evaporator-fan energy savings are much larger than the condenser penalty, so there is clearly a net benefit to the Advanced Controls retrofit.

- Integrated-economizer cases (temperature bins where economizer cooling must be supplemented by DX cooling) are processed so that when the DX is on, the fan speed corresponds to the capacity fraction of the unit. This approach attempts to satisfy the load by running part of the hour in economizer-only mode (high-fan speed) and part of the hour in DX-only mode. If first-stage DX (or minimum capacity of a variable speed system) can't sufficiently supplement the economizer, then the attempt at integrated operation is replaced by a normal DX analysis (no economizer). This reflects the fact that at higher stage levels for staged systems (or at capacity levels above the minimum capacity for units with variable-capacity condensers), the DX runs the whole hour.

- The calculation engine prevents any variable-speed system from operating in variablespeed mode below $\mathbf{1 5 \%}$ of capacity. When a variable-speed system operates below the $15 \%$ limit, it does not run the full hour. Instead, a runtime is calculated using the $15 \%$ minimum.

\subsection{Sensible AND LATENT LoAdS}

The following outline describes how the calculator accounts for latent loads. The progression here is from coil conditions to runtime calculations. Each of the three sections below begins with a brief statement or outline followed by a few paragraphs of explanation. Note that this content is also partially discussed in the previous section on "Equipment Response to Loads" (Section 4.7).

1. Evaporator Coil Conditions: Outside air (ventilation) and indoor air mix before passing over the coil. Conditions in the mixed stream depend on weather (outdoor dry-bulb and wetbulb) and indoor air (dry-bulb, setpoint, and room humidity).

The analysis of latent and sensible loads starts at the evaporator coil. It is the conditions at the coil that determine how much of the system's total capacity is available for handling sensible loads and how much of that capacity will be used for dehumidification. The coil conditions are determined by mixed-air calculations that consider the contributions from the two air streams (indoor return air and outdoor ventilation air).

2. Latent and Sensible System Capacity: Components of total capacity are based on mixed air conditions.

- Rated system capacities and S/T ratio (at AHRI test conditions) are projected to coil conditions.

- ASHRAE psychrometric routines (thermodynamic properties of moist air)

- DOE-2 total-capacity curves for evaporator coils

- EnergyPlus Apparatus dew point and bypass-factor algorithms for calculating latent and sensible components of total capacity 
Once coil conditions are known, then the rated capacities can be projected to them. This projection takes the characteristics of the system at AHRI test conditions (Outdoor dry-bulb $=95^{\circ} \mathrm{F}$, Inside dry-bulb $=80^{\circ} \mathrm{F}$, Inside wet-bulb $=67^{\circ} \mathrm{F}$ ) and projects to the mixed-air coil conditions in the simulation (as affected by indoor setpoint and weather-bin conditions).

The three sub-bullets list the algorithmic sources for the projection calculations. The result of the projection is sensible and latent capacities at coil conditions. A significant portion of the computational code of the RTUCC is in support of this step.

3. Runtimes are determined by the system's sensible capacity to maintain the indoor dry-bulb (satisfy sensible loads) as controlled by the thermostat.

- Building Sensible Loads: Load-line established to match (1) the system sensible capacity at outdoor design conditions and (2) the sensible internal loads when outdoor conditions equal the setpoint.

- Building Latent Loads (condensate): Calculated using S/T ratio at coil conditions and sensible loads (see "LLdE" and "S/T" columns in the bin tables).

The initial statement here on runtimes reflects how a thermostatically controlled air conditioner works. It simply tries to keep the dry-bulb temperature from rising. That is, as sensible loads act to increase the temperature of the room air (and eventually trigger the thermostat), the air conditioner runs (cooling the air) until the thermostat says to stop.

Runtimes are basically the ratio of the sensible load to the sensible capacity. So by establishing the sensible capacity of the system, we can determine how long it has to run (to satisfy the thermostat).

The sub-bullets on the sensible load-line describe the two outdoor conditions that establish the line (design and setpoint).

While the unit runs to counteract sensible loads, it also dehumidifies. To calculate condensate formation (latent coil load), the split between sensible and latent capacities (the S/T ratio) and the sensible load (SL), can be used. S/T is short for the ratio of the sensible to total capacities.

$\mathrm{LL}=\mathrm{TL} * \mathrm{~L} / \mathrm{T}=(\mathrm{SL} /(\mathrm{S} / \mathrm{T})) *(1-\mathrm{S} / \mathrm{T})$

This LL (Latent Load) is reported in the RTUCC as the "LLdE" column in the bin calculations. Another column that gives a good indication of how the coil conditions are affecting the sensible and latent capacities is the " $\mathrm{S} / \mathrm{T}$ " column.

Systems in humid climates will have a lower portion of their capacity available for sensible cooling (lower $\mathrm{S} / \mathrm{T}$ ratios). 


\subsection{ASSUMPTIONS ABOUT INDOOR HUMIDITY}

The RTUCC has two ways of modeling indoor humidity. The default is to assume that that indoor humidity ratio tracks with the outdoor humidity ratio. This assumption produces mixedair (coil conditions) more humid than would be seen in an hourly simulation like EnergyPlus. The corresponding latent coil loads will be higher in the RTUCC than a corresponding hourly model like EnergyPlus. The alternate assumption of fixed indoor humidity assumes that some other conditioning device is acting to control the indoor humidity to a fixed level. In this case, the latent coil loads of the RTUCC should be similar to that of an hourly simulation which also uses a fixed humidity assumption.

The data in Figure 4-9 was generated from EnergyPlus modeling of a medium-sized office building. This shows a positive difference during the summer cooling season (delta $\mathrm{T}$ greater than -10), and a negative difference in winter. Summer cooling, and resulting dehumidification, suppresses the indoor humidity ratio.
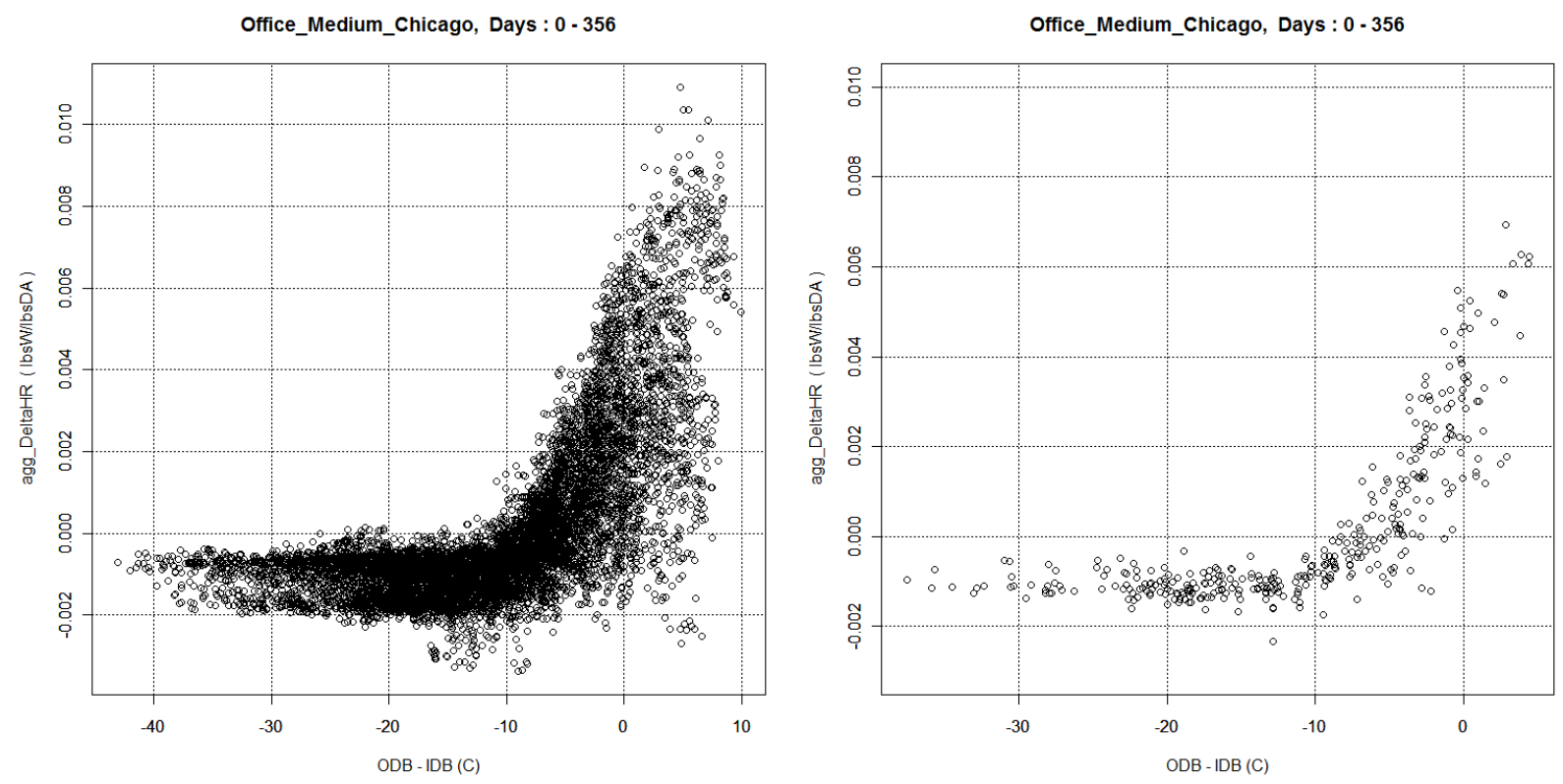

Figure 4-9 Humidity-ratio differential (outdoor minus indoor) as affected by temperature differential (outdoor minus indoor). Left: raw hourly data. Right: daily averages.

\subsection{ECONOMICS: EQUATIONS}

Economic results are presented based on the estimated annual costs and the purchase cost of the rooftop unit. The following equations are used in these calculations:

Uniform Present Value Factor: Factor used in calculating present value of a recurring cost.

$$
\mathrm{UPV}(\mathrm{N}, \mathrm{DR})=(\mathrm{a}-1) / \mathrm{DR} * \mathrm{a}
$$


where,

$\mathrm{a}=(1+\mathrm{DR})^{\mathrm{N}}$

$\mathrm{DR}=$ Effective Discount Rate (see next equation)

$\mathrm{N}=$ Number of years over which the recurring cost occurs

Effective Discount Rate: Discount rate after the effect of inflation is removed. Note this equation is not used in the calculator (the RTUCC's input for discount rate is an effective value, not a nominal value). The equation is offered here to further illustrate the difference between the nominal and effective discount rates.

$\mathrm{DR}=(1+\mathrm{ND}) /(1+\mathrm{IR})-1.0$

where,

$\mathrm{ND}=$ Nominal discount rate (including effects of inflation)

$\mathrm{IR}=$ Assumed inflation rate

Life-Cycle Cost: Present day value of purchase cost and annually recurring costs over $\mathrm{N}$ years of operation.

$\mathrm{LCC}=\mathrm{C}_{\text {purchase }}+\left(\mathrm{UPV}(\mathrm{N}, \mathrm{DR}) * \mathrm{C}_{\text {annual }}\right)$

where,

$\mathrm{C}_{\text {purchase }}=$ Unit purchase cost (purchase and installation)

$\mathrm{C}_{\text {annual }}=$ Annual cost (fuel and maintenance)

Annualized Cost: The uniform annual payment such that the discounted present value of the payment series is equal to the life-cycle cost of the system in question. Or it can be thought of as the uniform annual loan payments on a loan for the amount of the life-cycle cost.

$$
\mathrm{AC}=\mathrm{LCC} / \mathrm{UPV}(\mathrm{N}, \mathrm{DR})
$$

Net Present Value: The life-cycle cost difference between the two competing units.

$$
\mathrm{NPV}=\mathrm{LCC}_{\text {candidate }}-\mathrm{LCC}_{\text {standard }}
$$

Simple Payback: Estimate of number of years in which annual cost savings compensate for the additional capital costs of the candidate unit.

$$
\mathrm{SPB}=\text { Capital Cost Savings / Annual Cost Savings }
$$

Payback: Number of years in which annual cost savings compensate for the additional capital costs of the candidate unit. This is determined by iteration in the calculator (i.e., $\mathrm{N}$ when $\mathrm{NPV}(\mathrm{N})=0)$. 
Rate of Return: The discount rate at which the net present value is zero. This is determined by iteration in the calculator (i.e., DR when $\operatorname{NPV}(D R)=0$ ).

Savings to Investment Ratio: Ratio of the present worth of operating cost savings to the purchase cost savings.

$\mathrm{SIR}=\left({\mathrm{C} \_S T_{\text {annual }}}_{-} \mathrm{C} \_\mathrm{CA} A_{\text {annual }}\right) * \mathrm{UPV}(\mathrm{N}, \mathrm{DR}) /\left(\mathrm{C}_{-} \mathrm{CA}_{\text {purchase }}-\mathrm{C}_{-} \mathrm{ST}_{\text {purchase }}\right)$

where,

C_ $\mathrm{ST}_{\text {annual }}=$ Annual costs of standard unit

C $\mathrm{CA}_{\text {annual }}=$ Annual costs of candidate unit

$\mathrm{C}_{-} \mathrm{ST}_{\text {purchase }}=$ Purchase costs of standard unit

$\mathrm{C}_{-} \mathrm{CA}_{\text {purchase }}=$ Purchase costs of candidate unit

\subsection{ECONOMICS: CHARTING LIFE-CyCLE COSTS}

The chart in Figure 4-10 is produced when the calculator is run. It shows discounted (to present day value) costs for the candidate and base units, each year, over the specified system life. The cross-over point shows the payback time when the candidate unit's lower operating costs recover any difference in initial cost.

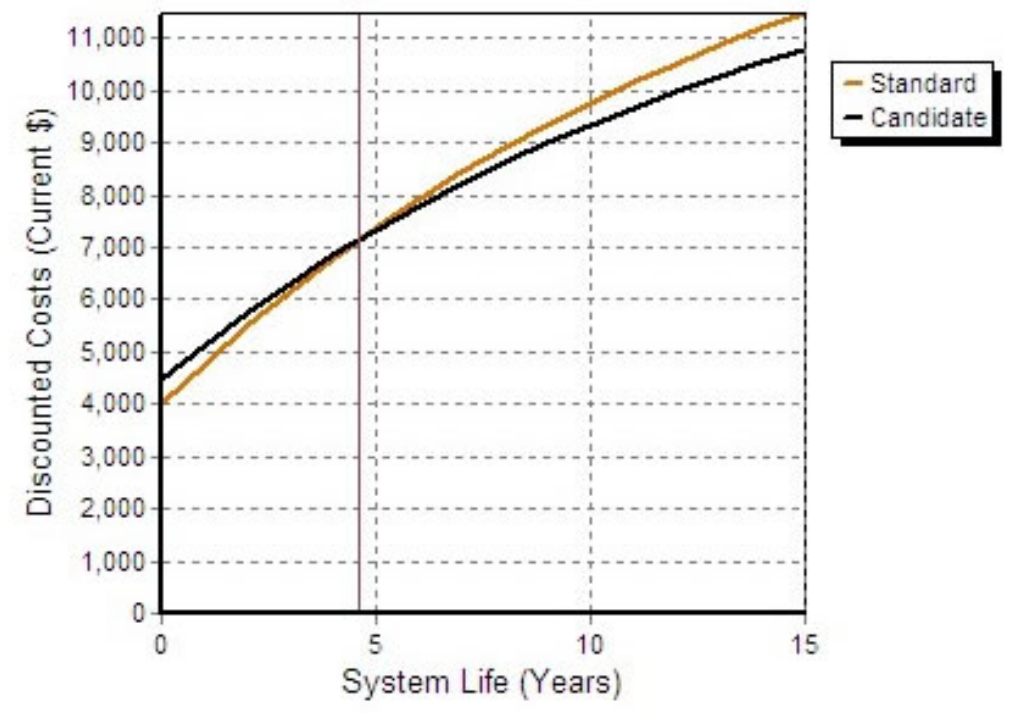

Figure 4-10 Example chart of discounted costs (Wichita, KS). 


\section{HELP TOPICS}

This section is a collection of help topics for each of the features on the calculator's Controls page. These help topics can be accessed by clicking on the question mark next to a feature's name. This opens the help window and positions the corresponding topic at the top. Topics are generally outlined as follows:

Name: The section name corresponds with the help-topic name (which is often the name of the feature).

Overview: Each section starts with a brief summary (or phrase) that is similar to the pop-up tip that is viewed by positioning the cursor over a question mark on the Controls page (and waiting one second or so).

Discussion: The remainder of the section includes a more detailed discussion. General tips on usage will be in the first part of this discussion. Following this may include additional discussion on the expected qualitative behaviors when changing the values of this feature.

\subsection{WEB BROWSERS}

The RTUCC has been tested and is fully functional in the Mozilla Firefox and Microsoft Internet Explorer web browsers. Performance and display characteristics are best in Firefox. In RTUCC version 4.3 and later, WebKit-based browsers such as Google Chrome and Apple Safari are now supported.

(In RTUCC version 4.2 and earlier, an issue with Chrome was apparent when returning to the Controls page from the Results page. The back button, or return links, could reset features on the Controls page to their default state.)

\subsection{Submitting AND SAVING}

Click one of the "Submit" buttons or any of the gray divider rows to submit the Controls page to the calculation engine.

The Controls and Results pages can be saved as local web pages to record run parameters and performance results. Note that a parameter-summary table is included on the Results page (after the "Results" table and chart).

Discussion: To capture the details of an RTUCC run, do a "save-page-as" from the browser and then pick the "web-page, complete" option. This works well from the Firefox and Google Chrome browsers. Google Chrome does the best job capturing all formatting details. Tables, images (graphs), and everything will be captured. (Note that currently the MS Internet Explorer browser does not properly capture the Results page.) From the saved local page, or directly from 
the original page, you can drag your cursor over tables and paste them into Excel for additional analysis.

The Controls and Results pages can also be printed to multiple sheets of letter size paper. Print from the "File / Print" menu option and not the "File / Print preview" option.

\subsection{Defaults}

Use the "Restore" button to change all feature values back to the defaults shown in the far right column. If a feature has been changed from its default value, the background color (of the corresponding table cell in this column) changes to a slightly darker shade. This color change is intended as an additional reminder to the user as to which features are actively being changed.

Most of the default values are static. However, some defaults are calculated (and updated) depending on the settings of other features. The "S\&I Fraction," "Ventilation Rate," and the three power features are examples of defaults that depend on other features.

The "Power" button is displayed next to the "Restore" button if the "Advanced Features" are turned on. The "Power" button acts to reset the power draw values. Clicking the "Power" button will cause the three power fields (evaporator, auxiliary, and condenser) to be set to default levels as calculated based on current settings of the "EER" and "Capacity" features. (See additional discussion in the help topic for the Power Inputs feature in Section 5.17)

\subsection{BUILDING TYPE}

Select the building type that best represents your building. Each building type has an associated load model that is used to predict RTU loads and mechanical ventilation as a function of outdoor temperature. Each load model is derived from EnergyPlus analysis on a prototype ASHRAE building.

Several parameters on this input form are calculated as the building type is changed (e.g. "S\&I Fraction" and "Ventilation Rate"). Manually entered values for "Ventilation Rate" will be overwritten whenever the building-type selection is changed.

To input custom load-model parameters, select the "Advanced Controls" option, then edit the parameter fields below the building-type feature. Refer to the help in Section 5.5 on custom load models.

If the load-line is locked, the building type (and two of the parameters in the custom-load model) will be locked (disabled). When locked, ventilation levels can be changed directly or by the ventilation parameter in the custom model.

Discussion: There are two supporting documents: (1) the description of the prototype buildings, see pages 8-13 in the PDF, and (2) a description of the corresponding EnergyPlus analysis done to develop the load models (Appendix A). 


\subsection{CuSTOM LOAD MODEL}

To define a custom load model, edit the three parameters and then click the "Apply" button. This calculates new values for associated features and must be done before submitting the form. (The "Advanced Controls" option must be selected to show the parameter fields for the custom load model.)

The parameter values for the custom model are initialized to the default parameters for the currently-selected building type.

When entering custom load-model parameters, the units are $\mathrm{KBtu} / \mathrm{hrF}$ for the slope (S), KBtu/hr for the intercept (I), and dimensionless for the ventilation-slope fraction (VSF). After editing these custom parameters, the "Apply" button must be used to calculate the corresponding new values for the "Ventilation Rate" feature.

If the load-line is locked (see Section 5.31), two of the parameters of the custom load model are locked (disabled) and cannot be changed. If locked, ventilation levels can be changed directly with the ventilation feature or with the ventilation parameter in the custom load model. The "Apply" button applies the ventilation levels set in the load model and overwrites any changes manually entered through the ventilation feature.

Discussion: A custom load model is defined to describe the approximate linear relationship between the sensible-cooling load on the heating, ventilation and air conditioning (HVAC) system and the difference between the inside and outdoor temperatures. The model is characterized by three parameters: (1) a slope parameter accounting for conduction, ventilation, and infiltration through the building envelope; (2) an intercept parameter accounting for internal loads; and (3) a ventilation-slope fraction parameter that specifies how much of the first parameter is caused by ventilation. Refer to the help section on building types for additional information. The load models documentation (see Appendix A) further describes the concepts and analysis behind this approach.

The slope and intercept of the load model are scaled to produce a sensible load that is in balance with the sensible capacity of the RTU at design conditions. In a sense, the building is scaled (sized) to match the capacity of the cooling system. This scaling operation produces the final load-line that is processed in the binned analysis.

\subsection{STATE/City}

The state feature affects the lists of cities appearing in the cities feature. The state and city features are used together to query weather data for this location. If your city is not listed, select the city whose climate is most similar yours (usually the closest city). 
Discussion: The calculator uses weather data to conduct a binned energy analysis for the RTU in cities across the United States. Weather tape data (outdoor dry-bulb and coincident web-bulb) was binned in 5 degree increments and filtered by the selected occupancy schedule. The result is a database of hours (in each bin) and coincident wet-bulb temperatures for each city and schedule combination.

\subsection{SCHEDULE}

This defines the time during which the building is assumed to be occupied. For the unoccupied periods outside of this schedule, the air conditioner is assumed to be shut down (no runtime) or the thermostat is set back. The system behavior during unoccupied periods is determined by the "Indoor Temperature" and the "E-Fan and Condenser" features.

Discussion: The "Schedule" feature filters the number of hours that can accumulate in a weather bin. Shorter occupant schedules translate into fewer potential hours where the cooling system can be subject to a load.

The hours outside of this schedule are considered to be unoccupied if the "Indoor Temperature" feature is set to "Cond. Off."

\subsection{INDOOR TEMPERATURE}

This is the thermostat setpoint. The second feature, "Setback," acts to increase the cooling setpoint temperature during unoccupied hours. The cooling setpoint, during unoccupied hours, is calculated as the sum of these two features (base setpoint + setback $=$ higher setpoint during the unoccupied hours). Additional information for this feature can be found in the topic for the "EFan and Condenser" feature (Section 5.19).

This setback feature acts to perform a double run. The load-line is established (locked) in the primary run (for occupied hours) at non-setback conditions. The same load-line is applied again in the secondary setback run for the unoccupied hours. The sum of the two runs is used to populate the energy categories in the "Results" table.

A special case is considered when "Setback" is set to "Cond. Off." In this case the condenser and the evaporator fan are not allowed to run during unoccupied hours and during these unoccupied hours, only auxiliary energy is calculated (and totaled). In this case, bin calculations are not displayed for the unoccupied hours.

For all normal setback cases, both condenser and fan energy (and auxilliary energy) are calculated during unoccupied hours. Turning on the "Show Bin Calculations" option will display separate bin calculations for these unoccupied hours. This is in addition to (displayed after) the occupied-hours bin calculations. (Note: there will be a link above each of the two "Loads and Hours" plots for the candidate unit. Clicking this link will scroll/jump the page back and forth between the occupied and the unoccupied-hours results. Just clicking this twice will put this jump into the browser's page history and then you can use the Alt-arrow keys (hold Alt key 
down, then try the left and right arrow keys) to toggle back and forth. This can be a useful trick for visually comparing the occupied and unoccupied results.)

Note that for staged systems using normal setback, the evaporator fan will cycle with the compressor during the unoccupied hours. However, if the special case of "Cond. Off" is selected, the condenser and the evaporator fan are always off during unoccupied hours.

The "Schedule" (scheduled hours) trace is shown in the "Loads and Hours" chart. This illustrates the occupied hours where the condenser is allowed to operate as established by the selected schedule. The "Total Hrs" trace shows all the hours (sum of occupied and unoccupied). Note that for normal setback, the "Schedule" trace in the "Loads and Hours" chart for unoccupied hours will represent the unoccupied hours (i.e., the hours where the condenser is active in the setback run for the unoccupied times).

Discussion: When interpreting the impact of changes to the thermostat setpoint feature, consideration must be given to whether the non-ventilation load-line is locked or not. If the loadline is locked, this feature will give the most intuitive results. However, in the default state (loadline unlocked), the impact of this feature may be less than expected. Consider the following example in Table 5-1:

Table 5-1 Candidate Unit Consumption as Affected by Indoor Temperature (locked and unlocked)

\begin{tabular}{c|c} 
Indoor Temperature & Candidate Unit (kWhrs) \\
\hline 75 & 10,798 \\
\hline 70 & 12,807 \\
\hline 70L (line locked@75) & 14,670 \\
\hline
\end{tabular}

Note: All other RTU parameters are at default values.

For the locked case (third row in table above), the primary driver behind the larger increase is that the non-ventilation load-line is not automatically adjusting to accommodate the more severe conditions at the lower setpoint (see discussion on locking feature in Section 5.31). As a result, the effect of the original non-ventilation load-line (based on delta-T) is clearly increased by the lower setpoint.

In the contrasting unlocked case (second row), the load-line is defined to be in balance with the capacity (see Methods page in Section 4.1). As a result, the change in setpoint has a less direct impact on the consumption. This is because the lower return temperatures adversely affect mixed-air conditions and the corresponding system performance. In addition, to achieve balance between total sensible load and the reduced capacity, the total sensible load must be reduced. Therefore, the primary driver behind the increase in consumption, for this case, is not directly loads, but rather seen most strongly in two associated effects:

1. Mixed-air conditions: The lower return temperature affects the load-line calculation (at design), the capacity (at bin conditions), and the power (at bin conditions) used while satisfying the load. The overall effect is an increase in power usage (see similar 
discussion of the unlocked case for the ventilation rate feature)

2. More cooling bins: The lower setpoint essentially extends the cooling season. There are more bins where the cooling loads are satisfied by the economizer but still add to energy consumption because of fan power.

Note that the setpoint affects the mixed-air conditions (for both locked and unlocked cases). The lower return temperature adversely affects the sensible capacity, which dominates the performance-correction effects (see the bin data for the overall correction factor (OCF)). The OCF factor is higher for the lower setpoint cases. Higher OCF factors correspond to higher consumption.

\subsection{INDOOR RELATIVE HUMIDITY}

Uncheck the "auto" feature to specify a fixed humidity level. In "auto" mode the inside humidity reflects outdoor conditions. In "fixed" mode, the indoor relative humidity is assumed to be controlled to a fixed value.

Discussion: The "auto" mode models the interior humidity as tracking the outdoor conditions. Tracking is equivalent to assuming a moisture mass balance if moisture gains (from people and processes) equal moisture losses from condensate. This approximation is a simple way to model humidity conditions if no other information is known about moisture dynamics in the building. The tracking mode calculates the interior relative humidity and wet-bulb by assuming the moisture content of the interior air is equivalent to that of the exterior air on a humidity ratio basis (i.e., mass of water per mass of dry air is equivalent inside and out). The tracking mode is constrained to allow indoor humidity of values no less than $20 \%$ and no more that $65 \%$. (The "auto" mode generally overestimates the interior humidity ratio. This assumption tends to overestimate associated latent loads. There is a discussion of this on the last page of the documentation on the building load models.)

Alternatively a "fixed" mode is used if the "auto" feature is not selected. In "fixed" mode, the interior humidity ratio is assumed fixed (at the user selected level) and is not affected by the outside conditions.

If you know the typical humidity levels in your building during the summer, use the "fixed" mode (especially if you have an associated piece of equipment that controls the humidity). If not, use the "auto" mode. Note that the calculator does not account for the energy usage of any associated humidification or dehumidification devices. It strictly calculates energy estimates for the air conditioner.

A comparison of empirical observations (in test homes) with these simplified methods for modeling humidity has shown acceptable agreement in annual estimates (Miller 1984).

Note that a sensible load analysis (also see Section 4.8) best represents a thermostat's sensible nature and is key in supporting the RTUCC's calculations that are affected by humidity and latent loads. A sensible thermostat responds to temperature changes and sends requests to the unit to 
remove the sensible cooling load. The effects of humidity are accounted for in how the unit's sensible capacity is diminished by humidity in the entering air (mixture of outside and return).

The change to a sensible analysis (in revision 2.0), from the original total-energy analysis, changes the question the calculation answers from:

\section{Total energy question}

Given the unit's total capacity at the specified entering conditions, AND assuming that all sensible and latent loads are met by the unit, how much energy would it consume in satisfying the total load?

\section{To:}

Sensible energy question

Given the unit's available sensible capacity at the specified entering conditions, AND that the unit is controlled by a thermostat (a purely sensible device), how much energy would it consume in satisfying the sensible component of the total load?

\subsection{Total CaPacity}

Select the AHRI net cooling capacity of the equipment being used (or equivalently, the cooling load of the building at design conditions).

If a variable-speed system is selected using the "E-Fan and Condenser" feature, the staging feature is ignored. In this case all the bin calculations are reported in the first stage.

Discussion: This is the total (sensible and latent) net (including evaporator fan energy) cooling capacity at AHRI test conditions.

In all cases (unless the spreadsheet interface is used), the units are represented with this AHRI rated capacity, the EER, and DOE-2 correction factors.

Note that changing the capacity value automatically changes the default power values for the blower fan and the condenser.

\subsection{NUMBER OF STAGES}

Set this calculator feature to reflect the number of stages (capacity levels) in the RTU. This determines whether the unit will process the load with one or multiple stages. A multiple-stage system minimizes part-load cycling losses and evaporator-fan energy (see Methods page on equipment response to loads in Section 4.7). Table 5-2 lists the assumed capacity levels for each of the five available staging levels.

Discussion: If a variable-speed system is selected using the "E-Fan and Condenser" feature, the "Number of Stages" feature is ignored. 
A two-stage unit with a single-speed fan can be modeled by setting the "Number of Stages" feature to "2" and the "E-Fan and Condenser" feature to either of the "1-Spd" options.

Table 5-2 Capacity levels for each "Number of Stages" setting.

\begin{tabular}{r|l} 
Stages & Capacity Levels \\
\hline 1 & 1.0 \\
\hline 2 & $0.5,1.0$ \\
\hline 3 & $0.4,0.6,1.0$ \\
\hline 5 & $0.2,0.4,0.6,0.8,1.0$ \\
\hline 10 & $0.1,0.2,0.3,0.4,0.5,0.6,0.7,0.8,0.9,1.0$
\end{tabular}

\subsection{OVERSIZING FACTOR}

Set this calculator feature to reflect the percent that the RTU's capacity exceeds load at design conditions. This factor serves to diminish the load so that the unit is effectively oversized at design conditions.

Blower-fan savings associated with variable-speed systems (see "E-Fan and Condenser" feature) are larger for oversized units.

\subsection{S/T RATIO}

The S/T ratio (the ratio of sensible to total capacity at ARHI test conditions) is used in estimating the sensible capacity of the unit. This along with the rated total capacity of the unit are the starting point for calculating sensible capacity and system power draw at conditions other than AHRI test conditions. See the Methods page on sensible capacity (Section 4.4) and also the interactive web page that calculates a table of steady-state system performance values at conditions other than AHRI test conditions.

\subsection{CANDidate Unit}

This row of features is used to set the Energy Efficiency Ratio (EER), cost (in k\$), and maintenance cost (in \$) for the candidate unit. Note that resetting the EER automatically changes the default power values for the condenser.

\section{Discussion:}

- EER: Net capacity / Total system power, as determined at AHRI test conditions.

- Cost: Total system purchase and installation cost (in units of thousands of dollars).

- Maintenance Cost: Total system annual maintenance costs (in units of dollars). 
Note that Integrated Energy Efficiency Ratio (IEER) values are not to be entered here. IEER values are a demographically and operationally weighted average of a set of four representative part-load EER values. An IEER value serves to project a unit's rated performance to a national level. These weighted averages are intended to rank units on a national basis. While IEER values are very useful in sorting units nationally, they are not intended for evaluating a unit for a particular climate and operation strategy. However, the raw EER part-load data that goes into the IEER calculation can be very useful for characterizing a unit's performance under specific conditions.

(Refer also to the help in Section 5.23 on the spreadsheet interface. The modeling of the full and part-load data is described there.)

The spreadsheet interface serves to adapt this supporting EER data (originally intended for the IEER calculations) for use in modeling work:

1. A superset of the four points: The 4 points are expanded to a table of 16 . These added points provide the information needed for building a regression model as a function of part-load fraction and outdoor dry-bulb.

2. Separation of evaporator and condenser power draws: Removing the evaporator fan power from the EER data set allows for independent modeling of the condenser unit and the evaporator fan.

\subsection{SPECIFIC CANDIDATE UnIT}

Select from a list of three specific high-performance RTU systems. Each of these three options corresponds to a commercially available rooftop unit or an add-on control system. The basic features are summarized below.

1. Advanced Controls: This is a retrofit fan controller that minimizes fan usage for any RTU that has a single-speed fan.

2. Three Stages: This is a high-performance RTU with three stages. Capacity levels are 40, 60 , and $100 \%$. This unit uses a three-speed evaporator fan.

3. Variable-Speed Compressor: This is a high performance RTU that uses a combination of staging, variable-capacity condensers, and a variable-speed evaporator fan. This unit first uses its variable capacity to meets the lower loads; at higher loads the additional fixed-capacity stages are used.

\section{Discussion:}

The setting of the "Setback" and "E-Fan and Condenser" calculator parameters significantly affects the assessment of the Advanced Controls system. Please also refer to the help topics for these two calculator parameters (Sections 5.8 and 5.19). 
The following outline lists the fan levels set by the Advanced Controls system. For two-stage RTUs, the first-stage cooling runs the fan at $75 \%$ and second-stage cooling runs the fan at $90 \%$. Single-stage RTUs address all calls for cooling by running the fan at $90 \%$.

- No call for cooling:

- Fan runs at $40 \%$

- Normal operation

- First-stage call:

- ODB $>=70^{\circ} \mathrm{F}$; fan at $75 \%$

- ODB $<70 \mathrm{~F}$; fan at $90 \%$

- Second-stage call: Fan at $90 \%$

- Economizer

- Fan at $75 \%$

- Fan at $90 \%$ (integrated)

Selection of either the "Three Stages" or the "Variable-Speed Compressor" RTU options invoke corresponding sets of capacity and efficiency correction curves for that unit. The "Advanced Controls" option only affects the behavior of the evaporator fan and therefore uses the default corrections curves.

Note that selection of a specific unit acts to configure associated features for the candidate unit. For example, selecting the "Three Stages" option sets the "Number of Stages" feature to "3" and the "E-Fan and Condenser" feature to "N-Spd: Always ON." Specific performance curves are used in the calculation engine depending on the selection.

However, a selection here does not automatically set either the "EER," "Total Capacity," or "Power Inputs" fields. It is necessary for the user to set these manually.

\subsection{STANDARD UNIT}

Use this feature to set the energy efficiency ratio (EER), cost (in $\mathrm{k} \$$ ), and maintenance cost (in \$) for the standard unit.

Discussion: See the discussion in Section 5.14 on the candidate unit.

Note that the standard unit's cost features can be used to investigate the economics of replacing an existing unit (with indefinite life remaining). In this case, the standard unit is not new and represents an existing unit. For example, starting with default settings set the standard (existing) unit to an EER of 8 and a cost of $\$ 1,000$ (assumed cost for an anticipated compressor replacement). Adjust maintenance costs to reflect the need for more annual service on the existing unit. 


\subsection{POWER INPUTS}

Specification of all the power inputs at AHRI rating conditions (in kWatts). This includes the evaporator fan (blower), auxiliary power (power needed for control electronics), and condenser unit (fan and compressor).

These three fields are initialized to default levels based on the total capacity and EER of the unit. Changing these three values will cause the EER to be recalculated. A helpful editing pattern is to first edit the "EFn" and "Aux" fields, then re-enter the "EER" value. Clicking the "Power" button (top or bottom right) will cause the three fields to be set to default levels based on current EER and capacity. Note that blower power can be determined from manufacturer's performance data as the difference between gross and net capacity when expressed in kWatts.

(Note that condenser-fan power can be specified with the "Condenser Fan" feature.)

The corresponding three categories of energy consumption are shown as columns in the bin-calcs table (the "Show Bin Calculations" option must be on). Their annual sums are shown in the "Results" table (the "Advanced Features" option must be on).

\subsection{CONDENSER FAN}

Use this feature to specify the condenser-fan power as a percentage of the total condenser power (at AHRI rating conditions).This feature has an effect on energy calculations ONLY in the case of variable-capacity condensers. This feature is disabled if the "E-Fan and Condenser" feature is set to a non-variable-speed mode.

Discussion: This feature acts to split out the condenser-fan power for use in accounting for the reduced-power draw of variable-speed condenser fans in the calculation engine. It has impact ONLY in the case of a variable-speed system. Fan affinity laws are used to estimate the reduced (from full-load) power consumption of the condenser fan. Full-load condenser-fan power is calculated using this feature's indicated percentage of the AHRI-rated condenser power (as specified in the "Cnd" field of the "Power Inputs" section).

\subsection{E-FAN AND CONDENSER}

Set this calculator feature to reflect the characteristics of the evaporator fan and the condenser. This feature is dual purpose in that it allows the user to select: (1) the fan type for single or multiple-stage systems or (2) a variable-speed system (i.e., variable-capacity condenser and a variable-speed evaporator fan).

The following options are available:

- Single-Speed Fan (1-Spd) for one or two-stage condenser:

- Always ON 
- Cycles with Compressor

- N-Speed Fan (N-Spd) for N-stage condenser:

- Always ON

- Cycles with Compressor

- Variable-Speed Fan for variable-capacity condenser (V-Spd):

- Always ON

The "Cycles with Compressor" fan modes will cycle the blower fan with the compressor during both occupied and unoccupied hours. In this case, the fan is completely off whenever the compressor is off. An exception to this occurs when the economizer is active. When economizing, the blower fan always runs at full speed.

The "...Always ON" fan-mode choices for staged units will cause the blower fan to be "...Always ON" during occupied hours, but will cycle the fan with the compressor during unoccupied hours. In other words, the "...Always ON" mode runs the fan at all times during occupied hours, but cycles during unoccupied hours. The fan is effectively in "Cycles with Compressor" mode during unoccupied hours.

The behavior of the system during unoccupied hours is affected by the "Setback" feature, which determines if the condenser is allowed to run during that time. When the "Setback" feature is set to "Cond. Off," then DX cooling and the evaporator fan are kept off during the unoccupied hours. When the "Setback" feature is set to any value other than "Cond. Off," then DX cooling is allowed during unoccupied hours and at loads as determined by a reduced thermostat setpoint.

A special case where the condenser and the evaporator fan are off during unoccupied hours can be investigated by setting the "Setback" feature to "Cond. Off." This will set everything off during unoccupied hours independent of how the "E-Fan and Condenser" feature is set.

(For clarity, the following two paragraphs restate the information above from a different perspective: condenser on or off.)

Blower-fan energy, when the condenser is off, is handled in three ways: (1) If a "Cycles..." fan mode is in effect, the blower fan is completely off when the condenser is off; (2) if a "...Always On" fan mode is in effect, the unit will reduce fan speeds if it can (e.g., an N-Spd or V-Spd fan) to levels that support ventilation; and (3) whenever the economizer is active, the fan runs at full speed.

Blower-fan energy, when the condenser is on, depends on the type of the unit, but basically the fan fraction goes up and down with the capacity fraction for any unit except those with singlespeed fans. The main exception to this is when the economizer is active; then the fan always runs at full speed.

The "N-Spd:" fan-mode choice is a valid option only when the unit has two or more stages. If the "Stages" feature is not set to "2" or higher, a warning message is displayed when the user attempts to "Submit" to the calculation engine. 


\section{Discussion:}

A noteworthy special case is a system with two stages and a single-speed fan. A single-speed fan system runs at one speed (full speed) during both first-stage and second-stage compressor operation. This system, if there are two stages, is assumed to have a row-split evaporator design (i.e., essentially two evaporator coils in series, one after the other, subject to the same air flow from one blower fan). The row-split design yields high sensible (less latent) performance when only the first-stage is active as compared to when both stages are active. In first-stage mode, the coil will be warmer and therefore yield a more sensible output (high fan flow coupled with lower condenser cooling causes warmer supply temperatures). This higher sensible capacity in the first stage can be seen in the calculator by looking at the $\mathrm{S} / \mathrm{T}$ values in the tables and charts for bins where the first stage is active without the second stage. Table 5-3 illustrates the variation in evaporator (blower) fan energy usage as affected by parameters in the calculator: (1) economizer mode, (2) setback mode, (3) condenser mode, and (4) fan mode. The results shown here are for the candidate unit. All parameters not represented in the table are set to default levels. Fan energies ran from the high of $3,167 \mathrm{kWh}$, to the low of 397 for a variable-speed fan that adjusts to reduce fan speeds when additional flow is not needed.

Generally, the table shows reductions in fan energy for systems that can reduce fan speeds when full flow is not needed. Also, the control option of allowing the fan to cycle with the compressor reduces the fan use and energy consumption. When the economizer is active, the fan savings of a "V-Spd" and "N-Spd" system are reduced (because the fan runs at full speed while economizing). The "1-Spd" fan consumes the most fan energy because it's always running at full speed.

It helps to consider a few specific comparisons in Table 5-3:

- Note that the 2,347 for the two-stage unit (in row 6) is higher than the 2,088 for the onestage unit (in row 5). Because of the setback mode, the condenser is active and the fan is cycling with it during unoccupied hours. The one-stage unit satisfies the load with shorter condenser runtimes (always running at full capacity). So there is more fan-only time (which means more time when the fan is off) during the unoccupied hours with the onestage unit. As a result, the one-stage unit uses less fan energy in this case.

- Contrast the above case with the corresponding two rows in the "Cond. Off" block of runs: 1,498 for both cases. The two systems yield identical results here because the fan is always on during occupied hours and always off during unoccupied hours.

Contrast two cases for the variable-speed fan: 397 (4th row) but 1,782 in the last row. The last run has more requirements for the fan to be running at full speed. As a result, the distinction between 1-Spd, 2-Spd, and V-Spd units in the last group of runs is smaller than the other three groups of runs. 
Table 5-3 Evaporator Fan Energy (kWhrs) as Affected by Operation Mode.

\begin{tabular}{l|l|l|l|l|l}
$\begin{array}{l}\text { Econ } \\
\text { Mode }\end{array}$ & $\begin{array}{l}\text { Setback } \\
\text { Mode }\end{array}$ & $\begin{array}{l}\text { Cond } \\
\text { Mode }\end{array}$ & $\begin{array}{l}\text { Fan } \\
\text { Mode }\end{array}$ & $\begin{array}{l}\text { Fan } \\
\text { Always On }\end{array}$ & $\begin{array}{l}\text { Fan } \\
\text { Cycles }\end{array}$ \\
\hline Off & Cond. Off & 1-Stage & 1-Spd & 1,498 & 771 \\
\hline Off & Cond. Off & 2-Stage & 1-Spd & 1,498 & 1,030 \\
\hline Off & Cond. Off & 2-Stage & N-Spd & 552 & 484 \\
\hline Off & Cond. Off & V-Spd & V-Spd & 397 & NA \\
\hline \hline Off & 5 deg. & 1-Stage & $1-S p d$ & 2,088 & 1,361 \\
\hline Off & 5 deg. & 2-Stage & $1-S p d$ & 2,347 & 1,879 \\
\hline Off & 5 deg. & 2-Stage & N-Spd & 794 & 726 \\
\hline Off & 5 deg. & V-Spd & V-Spd & 558 & NA \\
\hline \hline On & Cond. Off & 1-Stage & $1-S p d$ & 1,498 & 1,229 \\
\hline On & Cond. Off & 2-Stage & $1-S p d$ & 1,498 & 1,417 \\
\hline On & Cond. Off & 2-Stage & N-Spd & 958 & 953 \\
\hline On & Cond. Off & V-Spd & V-Spd & 756 & NA \\
\hline \hline On & 5 deg. & 1-Stage & $1-S p d$ & 3,167 & 2,898 \\
\hline On & 5 deg. & 2-Stage & $1-S p d$ & 3,283 & 3,202 \\
\hline On & 5 deg. & 2-Stage & N-Spd & 2,320 & 2,315 \\
\hline On & 5 deg. & V-Spd & V-Spd & 1,782 & NA
\end{tabular}

Capacity unloading can be achieved by multiple stages or other variable capacity methods. Consider a two-stage unit with a two-speed fan. A two-speed fan system has a lower fan speed when running only the first stage. Based on fan affinity laws, power consumption at the lower fan speed $\left(1 / 2\right.$ the air flow of the high-speed mode) is assumed to be one eighth $\left((1 / 2)^{3}=1 / 8\right)$ of the high-speed power specified in "EFn" field on the Controls page. Note: this feature is only visible when the "Advanced Features" are turned on. When only ventilating, the two-speed system runs at its lower fan speed.

The variable-speed choice represents a system that has a variable-speed evaporator fan and a variable-capacity condenser. Variable capacity is achieved either though many stages or other capacity-unloading methods. Detailed part-load condenser-performance behavior can be represented through the spreadsheet interface. The spreadsheet data represents the condenser fan and the compressor at part-load conditions and can be used to account for condenser fans that can reduce their speed. When either of the variable-speed options is selected, the "Stages" feature is effectively disabled and all bin results are reported in the columns for the first stage. The cycling-degradation factor is set to zero when either of the two variable-speed modes is selected; it is assumed that the variable-speed unit does not cycle.

Both variable-speed and staged-condenser systems run the condenser and evaporator fan at capacity levels needed to satisfy the sensible load. At reduced load, capacity and fan air flow are reduced together, yielding different sensible-latent splitting (as determined by the apparatus dew point and bypass factor method) of the total capacity. These systems are assumed to have either a row-split or interlaced evaporator design (serial air flow from one multi-speed fan). 


\subsection{DEgRADATION FACTOR}

Set the value of this calculator feature to represent the part-load degradation factor of the RTU as a percentage. This value determines how much the unit performance is degraded as a function of load fraction. At no load, performance is degraded by this percentage. At full load, the degradation is zero. A linear relationship is used for load fractions between these two extremes.

\subsection{1 $N$ AFFINITY}

This calculator feature sets the exponent in the fan-power calculations (fan-affinity laws). For example, if the fan runs at one half of its flow capacity, the power it consumes will be $(1 / 2)^{N}$ of its full-flow power draw. If $N$ is 3 , then at one half flow it will consume $(1 / 2)^{3}$ or $1 / 8$ of its fullflow power.

\section{Discussion:}

An $N$ of 3 is considered to represent the theoretical fan affinity law behavior for an ideal fan and yields a high-end estimate of the fan energy savings associated with a multi-speed fan. At lower fan speeds, this value is considered to overestimate fan savings. An $N$ value of 2.5 is typically accepted as an exponent that best represents overall fan behavior.

An $N$ of 3 was assumed for all RTUCC versions before 4.3.

Also see the discussion in the "E-Fan and Condenser" topic (Section 5.19).

\subsection{Demand Cost}

Use this calculator feature to set the monthly demand rate $(\$ / \mathrm{kW})$ and the number of applicable months.

The calculation engine keeps track of the peak power draw during the season. The demand charge is calculated as the simple product of this peak value and the rate and number of months values entered in the two fields. Demand costs are shown in the "Results" table if the "Advanced Features" option is on.

\subsection{SPREADSHEET DATA}

Use this feature to input detailed performance data from the spreadsheet available for download on the RTUCC website (use the download links in the help topic for this feature).

The purpose of the spreadsheet data is to facilitate detailed modeling of the full-load and partload performance of the RTU's condenser (compressor and condenser fan). The RTU's evaporator fan is explicitly modeled in the calculator and its part-load behavior is not characterized by the spreadsheet. Some characteristics of the RTU at rating conditions are 
imported from the spreadsheet and serve to populate some of the features in the calculator (e.g., $\mathrm{S} / \mathrm{T}$ ratio, power inputs...).

Note: the sample spreadsheet referred to in this topic can be downloaded in a zip file, which includes the DetailedPerformanceData_VSCD.xlsm spreadsheet. This spreadsheet contains Visual Basic (VB) code. To enable the V $B$ code (sometimes referred to as macros), the user will usually need to click a button titled "Enable Editing" and then a button titled "Enable Content" at the top of the Excel interface.

Copy (select with a mouse drag or use control-a to select and then control-c to copy) all data from the "Data Summary" sheet of a detailed specification spreadsheet and paste (control-v) it in the "Spreadsheet Data" text box. Then click the check box next to the text box. This feature is a mechanism to import detailed performance data to the calculation engine within the RTUCC.

The web application produces four regression models from the pasted data:

- Gross Total Capacity:

Function of ODB (outside dry-bulb) temperature and EWB (entering wet-bulb) temperature.

This is a relative model and depends on the nominal capacity value set on the Controls page.

- Condenser Power:

Function of ODB and EWB.

This is a relative model and depends on the nominal condenser power value set on the Controls page.

- S/T Ratio:

Function of ODB, EWB, and EDB (entering dry-bulb) temperature.

This is an absolute model and ignores the nominal $\mathrm{S} / \mathrm{T}$ value set on the Controls page.

- Part-Load Performance:

Normalized EER (excluding blower-fan power), as a function of load fraction and ODB. The EER data is first normalized by the first row in the part-load performance table (the $100 \%$ load EER). Therefore, data feeding this regression is relative to the full-load performance in the first row. This essentially captures the part-load behavior of the condenser (and its fan) and is used with the full-load condenser-power model described above. The part-load behavior of the evaporator blower fan is accounted for separately with fan affinity laws. The end result of this is a relative part-load model of the condenser, which ignores the nominal degradation factor set on the Controls page.

A sample spreadsheet is provided to illustrate and test the import feature. Right-click on the link and then choose to open or choose to save ("Save Link As...") the Excel file to your computer. Note that this spreadsheet is version marked to ensure compatibility with the web application. Paste operations from older versions will not be accepted.

This sample spreadsheet has two "scratch" sheets that illustrate how supporting data be can be found in PDF format on manufacturers' web sites and used for this feature. One of the scratch sheets shows the raw images of the supporting tables (from a Lennox PDF). The other sheet 
shows intermediate tables that were assembled by pasting data from the PDF into the spreadsheet.

The "Part-Load Performance" sheet has detailed part-load performance data that serves to characterize the part-load behavior of the condenser. The upper table of data on this sheet corresponds to the part-load data that is used to determine a unit's IEER value. As a confirmation of this, the cells on the diagonal of the upper table are used to calculate the unit's IEER (the IEER result is shown on this sheet). For modeling work, the off-diagonal cells in the table are needed to characterize the part-load behavior as a function of load fraction and the outdoor drybulb temperature. The lower table specifically represents the condenser unit and has the evaporator-fan power and evaporator-fan heat subtracted from the EERs in the upper table. These special EER-Cond values are needed to represent the condenser's part-load behavior (including the condenser fan). The equation for calculating EER-Cond is given on the "Part-Load Performance" sheet. Only the YELLOW cells on the "Part-Load Performance" sheet feed into the regression models. As mentioned above, the part-load behavior of the evaporator's blower fan is accounted for separately with fan affinity laws.

Note, however, that the "Part-Load Performance" data will almost never be available on a manufacturer's web site. In the future, fully populated spreadsheets, including the part-load data, may be available for download from the RTUCC site. These will be provided to us from manufacturers for a selected set of rooftop units. But for now, leave this data set to NA or try out the feature using the test data that is provided with the sample spreadsheet.

Steps for importing the spreadsheet data:

\section{Copy:}

Copy all the data on the "Data Summary" sheet. Using control-a is one way to select a whole sheet. Another is to click the little corner cell in the upper left, or simply drag with your cursor over all the active cells in the sheet. The part-load data (last four rows of the Data Summary sheet), may optionally be excluded if the manufacturer's part-load data is not available.

2. Paste:

First, turn on the "Advanced Features" on the web site (this will reveal the "Spreadsheet Data" feature). Paste this data into the appropriate "Spreadsheet Data" cell on the Controls page. There is one spreadsheet cell for the candidate unit and one for the standard unit. After pasting, the cell may appear empty (blank); not until after step 3 will there be visible text in the cell (so don't double paste). Note: to remove data from a "Spreadsheet Data" cell, put the cursor in the cell, then use control-a (to select all the content in the cell), then use control-x (to clear it).

3. Click the check box (just to the right of the spreadsheet field):

This sets controls to reflect the corresponding values in the spreadsheet (e.g., EER, net capacity, evaporator fan power, auxiliary power, condenser power, and S/T ratio). Note, after pasting spreadsheet data, the check box is checked automatically if you click anywhere (outside the spreadsheet cell) on the page. After clicking the check box, the model number of the unit will be displayed in the spreadsheet cell. 


\section{Submit:}

Click the "Submit" button. On the report page, you'll see the raw sheet contents parsed and reconstituted by the engine and displayed. There will also be a table for each of the four regression models that have been developed (the table indicates the model form and the model coefficients and their corresponding $\mathrm{T}$ values). If data is insufficient for modeling, a warning message will be displayed where the regression model table would normally be displayed.

After pasting in the data, the cell background color for the "S/T Ratio" and the "Degradation Factor" features will be a light yellow. This is a warning that these features are effectively disabled if the modeling process succeeds. The corresponding $\mathrm{S} / \mathrm{T}$ model and degradation model are absolute and do not use these values. It is best to think of the values for these features as backups that will be used if the corresponding part of the modeling process fails (or if insufficient data is provided). The report page will indicate which of the models have succeeded. Again, there will be corresponding warning messages for those that fail.

All the other features on the Controls page are still active and can be changed by the user. Note that by unchecking the check box (also see step 3 above), you are then free to edit the nominal values for any of the parameters brought in by the spreadsheet (i.e., EER, net capacity, blower power, auxiliary power, and condenser power). Re-checking the check box then brings back the nominal spreadsheet values and displays them on the Control page. Note that the "Power" (top right) button (see help for the power fields in Section 5.17) will uncheck this check box and set the power fields to their default levels; re-checking the check box will again display the values from the spreadsheet.

The regression models will be in effect as long as there is spreadsheet data in the spreadsheet field (regardless of the check box state). To remove data from a "Spreadsheet Data" field, put the cursor in the field, then use control- $a$ (to select all the content in the field), then use control-x (to clear it).

\subsection{VenTILATION RATE}

Use this calculator feature to specify the ventilation rate in CFM or as a percent of the fan capacity. Remaining fan capacity (not used in ventilation) is available for use by the economizer.

Ventilation rates are calculated automatically when a building type is selected. Ventilation rates are calculated to produce the same fractional contribution to the slope of load-line as was determined in a corresponding EnergyPlus analysis of these building types (see the "Building Type" feature in Section 5.4). Updating any feature that affects this calculation will cause the ventilation rate to be updated. Manually entered values are allowed but will only persist until a ventilation-rate update is triggered by changing the value of a related calculator feature (for example the "State/City" feature).

An alternate method for establishing the ventilation level is by adjusting the ventilation fraction in a custom building-load model (see the "Building Type" feature). 
When the "Ventilation Rate" feature is in the "\% of fan capacity" mode, the ventilation parameter can be set anywhere between $0 \%$ (entering mixed air is composed completely of return air) to $100 \%$ (entering mixed air is composed completely of outside air) of the fan's capacity. This parameter range is equivalent to changing the fresh air damper from fully closed $(0 \%)$ to fully open $(100 \%)$.

When in the "CFM" mode, values above $100 \%$ of fan capacity may be allowed. CFM values in excess of primary fan capacity are assumed to be supported by a secondary fan.

For both the "\% of fan capacity" and the "CFM" modes, ventilation values are not allowed to exceed levels where the non-ventilation load would be less than the internal load (i.e., a negative conduction load is not allowed; see the Methods page in Section 4.1). An error message is reported to the user in this case. Another way to look at this issue is to remember that the design load is calculated to match the design capacity and that as ventilation is increased, the nonventilation loads must decrease to maintain the match. However, ventilation levels are not allowed beyond the point where the non-ventilation load model would have a negative slope (an envelope better than a perfect insulator).

\subsubsection{Effect of location (city/state) on calculated ventilation levels}

You may notice that the calculated ventilation rates change as the city and state change; the milder the climate (i.e., cooler summer design temperature), the higher the ventilation rate. The milder climate causes the unit to have a larger effective capacity (better coil performance) at the cooler design conditions, and because the building auto-sizes to balance loads with capacity at design conditions, this mean higher loads from all contributing components, including ventilation. The building model assumes that ventilation contributes a certain fraction of the slope in the load model. This fractional contribution from ventilation is only dependent on building type and does not change with location. (This ventilation piece of the load pie can be observed in the numerical specification of each load model and also in the "loads-and-hours" bin-calc plots.) However, even though the fraction stays constant, the actual ventilation CFM will increase as the design loads are increased for milder climates. You can think of this as a larger building (to match the higher capacity) requiring more ventilation because it has more square footage.

\subsubsection{Impact of ventilation changes as influenced by competing effects}

As intuitively expected, increasing ventilation levels generally causes higher consumption (and higher savings) to be reported in the RTUCC. But there are several competing effects that can cause unexpected changes in consumption as ventilation increases. Settings of associated parameters in the RTUCC determine the relative impact of these competing effects. The outline following Table 5-4 discusses these associated parameters from within the context of a locked or unlocked load-line:

Table 5-4 contrasts the savings as affected by changes in ventilation levels (all other settings are at default values). The first row is the base case of $24 \%$ ventilation flow. The second row shows the results when the ventilation feature is manually set to $50 \%$. The third row shows the result 
when the RTUCC starts at a ventilation rate of $24 \%$, then locks the non-ventilation load-line, and then changes the ventilation to 50\%. A comparison of rows 1 and 3 illustrates that an expected increase in consumption can essentially be nullified if the ventilation is increased when the loadline is locked (see explanation below in the "locked" section). Higher ventilation rates (rows 4 and 5) show an expected increase in consumption.

Table 5-4 Candidate unit energy consumption as affected by ventilation rate (locked and unlocked load-line).

\begin{tabular}{c|l|c} 
& Ventilation rate & Candidate Unit (kWhrs) \\
\hline 1 & $24 \%$ & 10,798 \\
\hline 2 & $50 \%$ & 11,654 \\
\hline 3 & $50 \%$ (locked@24\%) & 10,777 \\
\hline 4 & $100 \%$ (locked@24\%) & 10,954 \\
\hline 5 & $200 \%$ (locked@24\%) & 12,758 \\
\hline \multicolumn{2}{|c}{ Note: All RTUCC parameters are at default values. }
\end{tabular}

If non-ventilation load-line is unlocked,

- As ventilation increases, the composition of the load changes but the overall load is still constrained to match the unit's capacity at design conditions. If it were not for the effect of ventilation on the mixed-air conditions, there would be essentially no effect by ventilation on consumption in the unlocked case. Put in other words, in the unlocked case, changes in consumption are not directly caused by changes in ventilation load; these ventilation changes have only a very minor effect on the total-sensible load-line. (As a result, the truncating effect, where there can be less fan energy because of fewer cooling bins, rarely happens in the unlocked case; this is described below for the locked case.)

- Mixed-air conditions may become more favorable to the load-line and system performance as ventilation increases. The mixed-air conditions (and how they affect the unit's sensible capacity and power draw) are predominately responsible for changes in consumption as ventilation levels change. Consider the three primary steps where the mixed-air conditions have influence:

1. The design point: The load-lines are based on the sensible capacity at the design point. Unlike when in locked mode, here the non-ventilation line is re-calculated with each parameter change. The weather conditions and ventilation rates (and corresponding mixed-air conditions) at the design point affect the sensible capacity at design and the resulting load-line.

2. The bin calculations of sensible capacity: Bin conditions determine how the equipment (sensible capacity) can respond (runtime) to the load-line.

3. The bin calculations of power draw: Bin conditions determine how much power is used while the system is running.

If non-ventilation load-line is locked,

- Fewer cooling bins and less fan energy: Cooling bins that have total sensible-cooling load less than zero are excluded from the analysis. Increasing ventilation can decrease the 
number of cooling bins (below the setpoint) because ventilation cooling satisfies the internal gains and results in no cooling load. This effectively shortens the cooling season for the unit. The shortening occurs in bins of minor internal loads where the fan-only mode is predominant (economizer is satisfying the load without much compressor on time). The result is that low temperature bins are often truncated from the analysis as ventilation increases.

- Oversizing strengthens this effect because when the system is oversized, fan energy accounts for a higher fraction of the total system energy use.

- Mixed-air conditions (from increased ventilation) may be favorable to system performance.

- Internal gains are better satisfied (in bins below the setpoint; especially true if the economizer is disabled):

\subsection{ECONOMIZER}

This economizer feature enables the calculator to simulate the use of an economizer. The economizer is simulated by effectively increasing the ventilation rate from the specified rate to $100 \%$ of fan capacity. If enabled, the economizer is represented to be activate in any weather bin where increasing the ventilation acts to decrease the air conditioning load.

\subsection{ELECTRIC UTILITY RATE}

Enter your local electric rate. If you do not know the rate, you can find it on your bill or call the local utility provider.

\subsection{EQUIPMENT LIFE}

This is the number of years of life before the RTU is replaced.

\subsection{NUMBER OF UNITS}

This is a simple multiplication factor used in calculating costs for a group of units.

\subsection{Chart Discounted Costs}

If checked, discounted purchase and operating costs are calculated (the present value). If unchecked, undiscounted costs are used (simple payback).

The discount rate is the rate ABOVE inflation (not the nominal discount rate that INCLUDES inflation).

Discussion: The discount rate is an interest rate that is adjusted to remove the effects of actual or expected inflation. Specifically, it is an interest rate that is used to calculate the present value of 
expected yearly costs excluding the effects of inflation.

Note that the discount rate in the RTUCC is not a nominal discount rate. Nominal discount rates include the effects of inflation. The discount rate is always lower than the corresponding nominal discount rate.

\subsection{Show Bin Calculations}

Select this feature to show the detailed calculations for each outdoor dry-bulb bin. Detailed results include engineering calculations on system loads and performance, are tabulated and charted by outdoor dry-bulb bin temperature.

\subsection{LOCK LOAD-LINE}

Select this calculator feature to preserves the current non-ventilation load-line for use in any submits that follow.

Locking the non-ventilation load-line is equivalent to fixing the characteristics of a fictitious building that is the source of the cooling loads. Locking causes loads that are associated with internal gains to be fixed. Loads associated with conduction through the envelope are still driven by weather conditions, but the relationship to the temperature differential is fixed (slope of the load-line is fixed). Ventilation loads can still be adjusted after locking either by directly editing the ventilation value or by changing the building type or the ventilation parameter of a custom load model.

Discussion: In the default (unlocked) mode, loads are calculated to balance the unit's capacity at design conditions. Total sensible loads automatically adjust to balance capacity (also see the Methods page in Section 4.1 on the non-ventilation load for additional discussion). This principal assumption in the calculator can lead to counterintuitive (but correct) results in some of the features. For example (see Table 5-5), starting from the humidity feature's default "fixed mode" (not auto mode) value of $60 \%$ relative humidity, and then changing to $40 \%$, results in a significant drop in consumption if the load-line is locked (when at the original value of $60 \%$ humidity). A strong drop results when the load-line is locked because sensible non-ventilation loads stay fixed as sensible capacity increases at lower humidity levels. The result is lower runtimes.

Table 5-5 Candidate Unit Consumption as Affected by Humidity Levels (locked and unlocked)

\begin{tabular}{l|c} 
Inside Relative Humidity (\% R.H.) & Candidate Unit (kWh) \\
\hline 60 & 11,074 \\
\hline 40 (line locked @ 60) & $\mathbf{9 , 9 3 2}$ \\
\hline 40 & 11,190 \\
\hline
\end{tabular}

Note: All RTUCC parameters except "Fixed" inside relative humidity are at default values. 
Locking the non-ventilation load-line can be useful in testing the sensitivities to a parameter. Locking changes the "question" that is presented to the calculator. Consider, for example, the ventilation level parameter:

A. If locked, the question is: For fixed building characteristics, what is the energy impact of changing the ventilation level from:

1. an initial state where loads and capacities balance at design conditions,

2. to a new state (different ventilation) where total loads are free to be higher or lower (non-ventilation load is locked) than capacity at design?

B. If not locked, the question is: For an "adjustable" building that can adapt to be in balance with loads, what is the energy impact of changing the ventilation level from:

1. an initial state where loads and capacities balance at design conditions,

2. to a new state (different ventilation) where loads and capacity are again constrained to match and where the matching is again achieved by adjusting the non-ventilation load (the conduction component of the "adjustable" building)?

Or more simply put, once in locked mode, the non-ventilation building characteristics are fixed. When not locked, the "adjustable" building adjusts (conduction loads) to achieve balance between the specified ventilation load and the capacity of the unit.

Note: when using the locking feature, it may be best to use some "oversizing" to allow for extra capacity to accommodate load changes. The oversizing feature can help to minimize the number of bins where runtimes exceed 1.0. When locked, runtimes exceeding 1.0 are highlighted with light yellow background.

\subsection{APD/BPF}

This topic refers to the Demonstration web page for the Apparatus Dew Point and ByPass Factor Method (Carrier et al. 1959). A link to this web page is available on the Methods web page titled "Corrections to Tested Performance: Sensible Capacity."

Discussion: The first row of parameters serves to establish the capacity, flow rate, and sensible to total ratio (S/T) at AHRI rating conditions. These can be used to calculate the corresponding bypass factor at AHRI conditions. The bypass factor can be projected onto other mass flow rates using the expression $B F=\exp (-A 0 /$ massflowrate $)$. $A 0$ can be determined at AHRI conditions and then the expression can be applied at other mass flow rates. The second row of parameters, capacity and air flow rate, establish a baseline for a projection exercise, as illustrated in the output table below the parameters table. The table displays $\mathrm{S} / \mathrm{T}$ results for a variety of operating conditions, all at the capacity and flow rate established in the second line of parameters, all dependent on the $A O$ term established by the first row of parameters. By default the second line is set to equal the first line whenever a first-line parameter is changed. 


\subsection{AdVANCED FEATURES}

Use this feature to unhide the advanced-calculator features and initialize them to their default values. Advanced features are indicated by a tan background color in the features title cell. "Advanced Features" were new with the second release of the calculator. The new features in that release and those after are listed in the calculator's revision history. 


\section{REVISION HISTORY}

Version 4.3 (10/09/2014)

- Revised and updated the calculation engine to support the assessment of three specific high-performance RTU systems (each of these options corresponds to a commercially available add-on control system or a specific rooftop unit).

- A new calculator feature, "Specific Candidate Unit," allows the user to select one of these specific systems for evaluation. Selecting a system activates specific algorithms to represent the system:

- Specific fan-control strategies from the manufacturer of the advancedcontrol system.

- Specific performance curves from the manufacturers of the three-stage unit and the variable-speed unit.

- Extended general algorithms for modeling staged systems to represent units having three or more stages.

- Extended algorithms for modeling variations in evaporator fan speed, condenser fan speed, and condenser cooling capacity to better represent staged and variablecapacity RTUs. This yields better estimates of sensible capacity and better modeling of row-split evaporators as affected by flow and capacity variations. This also improves the calculator's estimates of evaporator and condenser fanenergy consumption and better estimates fan energy when a system is in economizer mode.

- Added a feature to specify the $n$ value used in applying fan affinity laws in fanenergy calculations.

- Promoted the "Number of Stages" feature so it can be specified differently for the candidate and standard units.

- Updated and added content to the Help and Methods pages. Added a new quickstart page that is accessible from the main menu. Added an overview page to the spreadsheet and additional annotation.

- Added functionality to support the calculation of savings related to variable-speed condenser fans. Split out the condenser fan on the Controls page, calculation engine, and Results page. Added a "Condenser Fan" help topic and updated related help topics and Methods pages.

- Updated the spreadsheet to include example calculated values in the "EER" and "EERCond" part-load tables on the "Part-load Performance" sheet. This is done with Visual Basic code embedded in the workbook. The code includes regression models for capacity and condenser power for an example RTU represented by the spreadsheet. This code also accounts for common part-load behaviors: (1) variable-speed condenser and evaporator fans, and (2) cycling degradation.

- User interface improved:

- Added page-state capture and re-load (from local storage) capability on the Controls page to support use of WebKit-type browsers such as Google Chrome and Apple Safari. This is necessary to preserve the state of several of the features when returning to the Controls page from the Results page. 
- Added a feature to preserve the vertical scroll position of the Controls page. This preserves the scroll position when using features that invoke a resend (regeneration) of the Controls page from the server.

- Modified the custom-load model features to enable viewing the load-model parameters for each of the standard building types. Modified the lock-the-loadline feature so that locking now acts to disable the "Building Type" feature and two of the associated custom-model features. Added a help topic for the customload model features.

- Changed the humidity feature to be visible whenever "Advanced Features" is selected. This is consistent with the approach used with the custom-load model features.

- Resolved several Cascading Style Sheets (CSS) formatting issues to improve rendering behavior and speed in the Internet Explorer and Google Chrome browsers.

- Added an input parameter-summary table that is displayed after the results table for each run.

- Combined the two discounting-related features into one row.

- Removed the Solar and Internal Gains (S\&I) feature, which has been replaced by the "Building Type" feature. The S\&I fraction is now reported in the design table as the "Internal Loads Fraction" in the "Design Conditions" table.

- Made each gray-divider row on the Controls page to act like a submission button.

- Improved the x-axis and y-axis scaling on the "Loads and Hours" and "Equipment Performance" charts so that all four charts (standard, candidate, occupied, and unoccupied) are scaled similarly.

\section{Version $4.2(9 / 26 / 2012)$}

- Corrected a problem in the fan energy calculations. This issue occurred when the "Fan and Compressor" feature was set to "V-Spd Always ON" or "2-Spd Always ON" and the "Setback" feature was set to "Cond. Off." For these two cases, the calculator overestimated fan energy consumption during unoccupied hours.

\section{Version $4.1(9 / 21 / 2011)$}

- Implemented the use of EnergyPlus load models to calculate S\&I factors and ventilation levels based on the selection of a building type.

- Changed the web application name from UAC Cost Estimator to RTU Comparison Calculator (RTUCC).

- Updated the Help and Methods pages. Added new content on latent-load calculations and the building-type feature.

\section{Version $4.0(1 / 15 / 2011)$}

- Apparatus Dew Point / By-Pass Factor (ADP/BPF) modeling of sensible capacities (S/T ratios): 
- Full implementation of iterative solution methods. Robust approach for web application. New module with shared psychrometric routines and ADP/BPF functionality.

- Dynamic web-based regression capability:

- Implemented commercial module in the calculator. Applied this for a dynamic regression of detailed user data and $\mathrm{ADP} / \mathrm{BPF}$ demo page.

- Restructuring of software to better support double runs of the engine (full comparisons) and promotion of general features to the specific feature groups (standard and candidate):

- Classes and procedures for structuring of bin data and parsing and modeling of spreadsheet data.

- Fan controls and algorithms:

- Collected new supporting fan data for fan power (vs. capacity) for setting power defaults.

- Converted to more appropriate (industry standard) curve shaping methods where operations are done using gross capacities (not net).

- Features for user specification of evaporator fan, condenser unit, and auxiliary electronics power draw.

- Choices for user-control strategies of the fans and compressors including simulation of variable-speed fans and variable-capacity compressors.

- Fan affinity laws are used in calculating fan power at part-load conditions.

- Spreadsheet interface:

- Added feature for importing detailed full-load performance data from a spreadsheet into the web application. Built data processing capability in the web application for modeling (regressing) and using the detailed performance data from the specification spreadsheet. This is a mechanism to feed data from a manufacturer's detailed specification into the calculation engine of the web application.

- Added feature for importing and modeling condenser part-load performance based on a spreadsheet table of part-load EER values. This table is a superset of the part-load EERs that are required to calculate an IEER aggregate.

- Night setback:

- This feature supports an automated double run of the calculation engine. The double run splits operation into occupied and unoccupied bin hours. Unoccupied hours are analyzed at a setback temperature reduced from the setpoint used during occupied hours.

- Oversizing feature:

- Changed to a more intuitive user interface and supporting algorithms.

- Payback calculations:

- Improved the calculation approach and corresponding messaging to the user.

- Larger range of capacities (3 to 30 tons) and corresponding additional DOE-2 shaping curves added for units less than 5 tons.

- User specification of system performance degradation as a function of load fraction.

- Features and algorithmic support for demand (peak power) and corresponding cost calculations.

- Promoted some existing features so they can be configured separately for each unit: economizer, $\mathrm{S} / \mathrm{T}$ ratio, degradation factor. 
- Improved mixed-air routines for better accounting of fan heat (mixing based on mass flows).

- Correction of load-matching problem at design conditions.

- Indoor humidity limits.

- Various improvements in the report page to support new features.

\section{Version $3.1 \quad(11 / 16 / 2004)$}

- Added hover-help tips for when mouse cursor is over the defining table cells of the "Design Conditions" and "Bin Calculations" tables. This includes any table cell with a cryptic definition in the bin-calcs report. Made some minor changes to the definitions on the bin-calcs report page.

- Added restriction to inside humidity ratio calculation (when in auto mode) so that its corresponding relative humidity cannot exceed $100 \%$.

- Removed feature that automatically fed manufacturer-specific cost data to the cost features.

\section{Version $3.0 \quad(04 / 10 / 2003)$}

- Changed economics algorithms

○ Discounting: Changed the uniform present value equation to be dependent on the effective discount rate (as opposed to using the nominal discount rate, an assumed inflation rate, and an escalation rate as was done in the original release).

- Rate of Return: Changed from an approximate closed-form equation to an exact iterative method.

- Payback: Added feature to calculate and present an exact iterative result.

- Added Methods pages and context-sensitive help topics.

- Added capability to represent special performance characteristics of manufacturers' units.

- Changed default value of S/T ratio (an "Advanced Feature") from 0.75 to 0.72 . Note that this change slightly affects the results as compared to Version 2.0. To reproduce Version 2.0 results, simply set the $\mathrm{S} / \mathrm{T}$ feature back to 0.75 .

- Added a "retail" schedule: M-Fri 9am-10pm, Sun 11am-6pm.

- Added feature to track if feature values differ from the defaults (background color of the default table cell turns yellow if selection differs from default value).

- Changed the definition of the Overall Correction Factor (OCF) factor to include normalization by the $\mathrm{S} / \mathrm{T}$ ratio at AHRI test conditions.

Version $2.0(10 / 17 / 2002)$

- Converted the calculation engine from a "total" to a "sensible" energy analysis (see help topic on "Advanced Features").

Note that this change significantly affects the results from the Unitary Air Conditioner (UAC) Cost Estimator. Refer to the discussions in the help topics for additional information on qualitative behaviors associated with various features (see especially: ventilation, humidity, lock load-line, and enthalpy controls)

- Added "Advanced Features" feature. 
- Added mixed-air calculations to properly determine the conditions entering the evaporator coil.

- Added a system performance calculation page that serves to illustrate the correction methods (see Methods pages: system power draw and sensible capacity). It also demonstrates the iterative apparatus dew point methods that were used in developing the generalization to the sensible capacity correction. This generalization serves to extend the DOE-2 curves to entering temperatures other than $80^{\circ} \mathrm{F}$.

- Corrected formulation error related to oversizing factor.

- Corrected formulation error related to staging.

\section{Version $1.0(09 / 28 / 2001)$}

- Initial release 


\section{REFERENCES}

EnergyPlus Engineering Reference, 2014. The Board of Trustees of the University of Illinois and the Regents of the University of California through the Ernest Orlando Lawrence Berkeley National Laboratory.

Carrier, W.H., R.E. Cherne, W.A. Grant, and W.H. Roberts. 1959. Modern air conditioning, heating and ventilating, $3 \mathrm{~d}$ ed. New York: Pitman Publishing Corporation.

Wang W, S Katipamula, H Ngo, RM Underhill, DJ Taasevigen, and RG Lutes. 2013. Advanced Rooftop Control (ARC) Retrofit: Field-Test Results. PNNL-22656, Pacific Northwest National Laboratory, Richland, WA.

Miller, J.D., "Development and Validation of a Moisture Mass Balance Model for Predicting Residential Cooling Energy Consumption," ASHRAE Transactions, Vol. 90, pt. 2, 1984, (275-293)

Miller, J.D., W. Wang, and S. Katipamula. 2014. RTU Comparison Calculator Enhancement Plan. PNNL-23239, Pacific Northwest National Laboratory, Richland, WA. 


\section{APPENDiX A: BUILDING RESPONSE MODELS}

This appendix has the following objectives: (1) to illustrate the concept of the linear response model (Section A.1) and explain how these building models will be implemented in the RTUCC, (2) to demonstrate how this response data can be extracted from EnergyPlus runs, (3) to justify a simplified representation method, and (4) to document the supporting EnergyPlus runs and postprocessing analysis.

\section{A.1 InTRoduction}

The RTUCC uses a binned-weather analysis to estimate building loads and predict a corresponding air conditioning system's energy use. A fundamental part of this analysis is a linear model (see Figure A-1) that is used to represent the building's thermal response to outdoor temperature and its internal loads.

The linear-response model predicts the thermal load on a building's cooling system as affected by the temperature differential between the outdoor and indoor temperature. This response reflects the nature of the physical building and its internal loads.

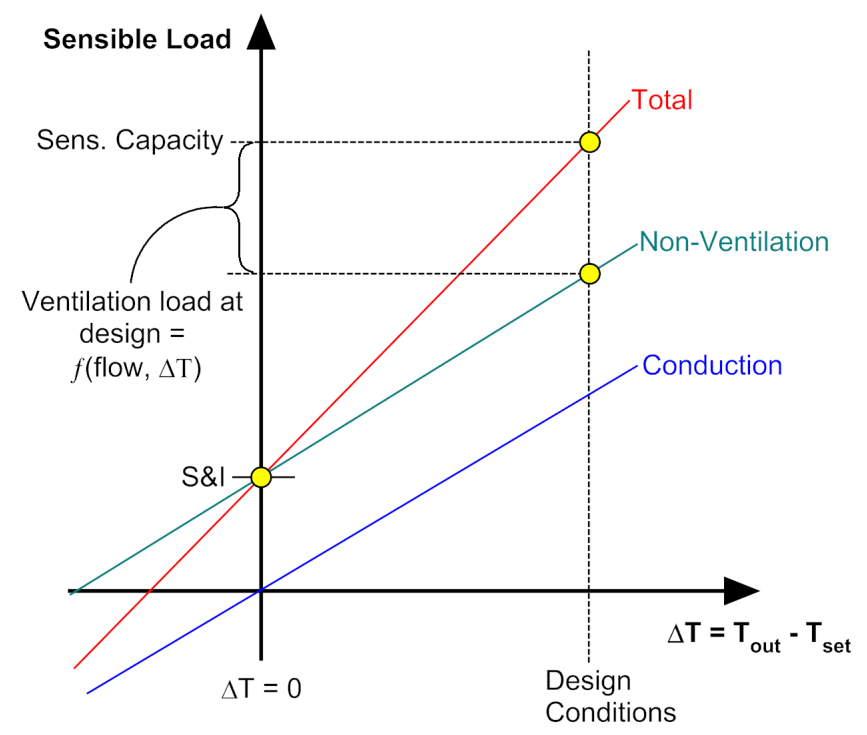

Where S\&I are the SOLAR and INTERNAL gains

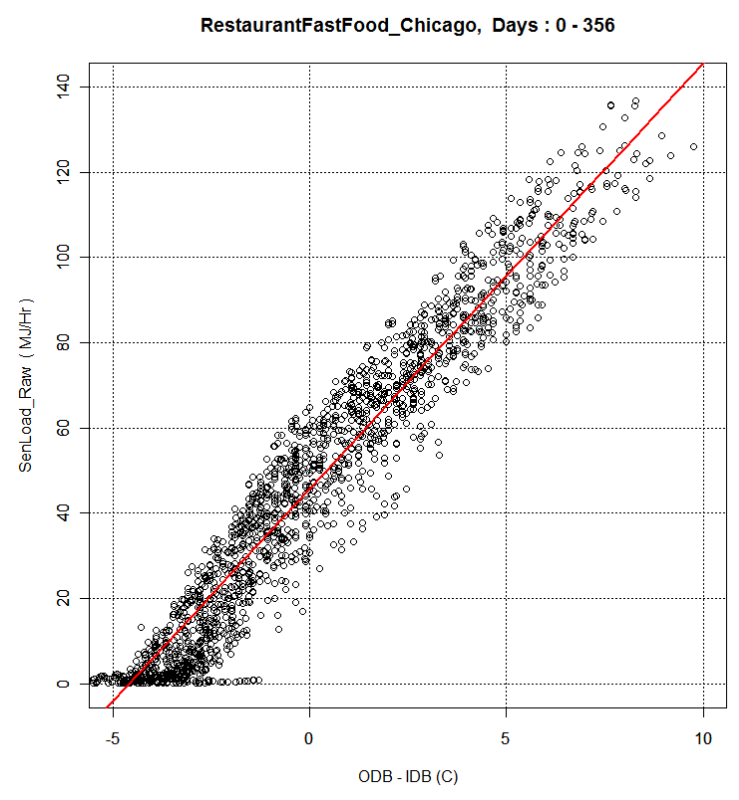

Figure A-1 Sensible load as affected by envelope temperature differential. Left: Load-line concept drawing. Right: Corresponding example of load data from an EnergyPlus simulation.

The analysis in this report serves to establish the slope and intercept of the load-line (see red lines in Figure A-1). This is done by modeling the aggregate sensible load as a linear function of the indoor-outdoor temperature differential. 
These response models are shown in this report to be approximately independent of the weather data driving the EnergyPlus runs. This means that one response model can be used to represent one building type at a variety of locations. Hotter climates will have more operation hours at higher delta-T, but the slope and the intercept will be similar to those seen in cooler climates.

A simple numeric indicator of the interaction between building type and climate can be determined from a calculation of the S\&I fraction. This fraction, as affected by the city's design temperature, is defined in the RTUCC as follows:

$$
F_{S \& I} \equiv \frac{T S L(\Delta T=0)}{T S L\left(\Delta T=T_{\text {design }}-T_{\text {set }}\right)}
$$

Equation A-1 states that the S\&I fraction is defined to be the ratio of the total sensible load (TSL) at neutral conditions, i.e., when outdoor temperature equals the indoor setpoint, to the TSL at design conditions. This can be visualized in Figure A-1 as the ratio of the loads at the two yellow dots on the red total-sensible load-line.

At neutral conditions, the load is primarily caused by solar and internal effects (this is the motivation for the S\&I name). A particular building design operating in a cooler climate will have a higher S\&I fraction than the same building running in a hot climate. One response model, for one building type, predicts a different S\&I fraction for each climate, depending on its design temperature. As expected, internal loads are a larger fraction of the design load in cooler climates.

The S\&I fraction and the unit's sensible capacity at design are two numbers from which a cityspecific total-sensible load-line can be formed. This calculation effectively scales the response model to match the sensible capacity of the RTU at the city's design conditions. This scaling process preserves the ratio between the slope and intercept of the response model. (Note that this use of the S\&I fraction in the scaling process comes from earlier versions of the RTUCC, which required the user to enter an S\&I fraction to specify the building load characteristics. This scaling process, which uses the $\mathrm{S} \& \mathrm{I}$ factor as an intermediate step, is completely equivalent to directly scaling the load model to match capacity at design. This equivalence can be verified in the RTUCC by comparing a non-ventilation version of the load model on the Controls page with the non-ventilation load-line that is reported on the Results page. The two slopes and the two intercepts are related by a simple scaling factor.)

An implicit assumption in this approach is that the response model scales simply with building size. "Simple scaling" means that the two parameters in the model, the slope and the intercept, both scale at the same rate as the building size increases or decreases. Improvements on this "simple scaling" assumption could possibly be incorporated by considering that internal loads and ventilation loads may scale with floor area and that the conduction loads may scale with external envelope area. The geometry of the building could be used to establish different scaling factors for the two model parameters.

This "simple scaling" assumption means that the total (including ventilation) sensible-load behavior of the building can be captured or represented by the ratio of the intercept and slope 
values of each linear model. This intercept to slope ratio ("I/S") is reported below in Table A.1. This single "I/S" number is sufficient representation; however, for clarity both the slope and the intercept values are used and displayed in the RTUCC.

An additional characterization of the building is done by modeling the ventilation-sensible loadline. The slope of this line can be compared to the slope of the total-sensible load-line. The ratio of the two slopes (ventilation-sensible-load slope / total-sensible-load slope) is a good indicator of the factional contribution of ventilation in the load-line. If this ratio is 0.6 , it means $60 \%$ of the temperature-dependent response is attributable to ventilation. This ratio is the third parameter that will be used to characterize a building type. This parameter (for a type of building) and a city's design conditions are used in the RTUCC to establish default ventilation rates.

Ideally a sensible-load model is preferred. Sensible models best reflect how a direct expansion (DX) system works to remove sensible loads as dictated by a sensible thermostat. It will be shown in this report that total-load models make reasonable substitutes for the preferred sensible models, especially with building simulations where the sensible loads are not available. 


\section{A.2 SuMmary OF FINDINGS}

- Variation between response models, by building type, is strong enough to justify a representative model for each building type.

- A building response model developed for Chicago is sufficient for representing similar buildings in different climate zones.

- Total (latent + sensible) and sensible load data produce similar results when developing load models.

- Models can be developed without turning off setback or ventilation in the building. Modifications to EnergyPlus Input Data Files (IDF) are only needed to change reporting variables.

Results from each EnergyPlus run are summarized in Table A.1. "Slope" and "Intercept" columns are shown in both metric (columns 3-5) and English units (columns 6-8). The "I/S" columns are the ratio of the intercept to the slope and are an indicator of the degree of internal loading in the structure (high for hospital, low for warehouse). The "Ventilation Fraction" column is the fraction of the slope in the load model that is attributable to ventilation. The "Assumed VF" column indicates an assumed ventilation-fraction value that is currently being used in substitution of the run result.

Table A.1 Summary of Run Results.

\begin{tabular}{|c|c|c|c|c|c|c|c|c|c|}
\hline & Building Type & $\begin{array}{l}\text { Slope } \\
(\mathrm{MJ} / \mathrm{hC})\end{array}$ & $\begin{array}{l}\text { Intercept } \\
(\mathrm{MJ} / \mathrm{h})\end{array}$ & $\mathrm{I} / \mathrm{S}(\mathrm{C})$ & $\begin{array}{l}\text { Slope } \\
\text { (KBTU/hF) }\end{array}$ & $\begin{array}{l}\text { Intercept } \\
\text { (KBTU/h) }\end{array}$ & $\mathrm{I} / \mathrm{S}(\mathrm{F})$ & $\begin{array}{l}\text { Ventilation } \\
\text { Fraction }\end{array}$ & $\begin{array}{c}\text { Assumed } \\
\text { VF }\end{array}$ \\
\hline 1 & Mid-Rise Apartment & 12.3 & 128.3 & 10.4 & 6.5 & 121.6 & 18.7 & NA & 0.30 \\
\hline 2 & High-Rise Apartment & NA & NA & NA & NA & NA & NA & NA & ------ \\
\hline 3 & Hospital Healthcare & 55.8 & 1370.0 & 24.6 & 29.4 & 1298.5 & 44.2 & NA & 0.80 \\
\hline 4 & Outpatient Healthcare & 41.8 & 704.0 & 16.8 & 22.0 & 667.3 & 30.3 & 0.05 & 0.25 \\
\hline 5 & Small Hotel & NA & NA & NA & NA & NA & NA & NA & ----- \\
\hline 6 & Large Hotel & 149.5 & 1313.0 & 8.8 & 78.7 & 1244.5 & 15.8 & 0.96 & 0.60 \\
\hline 7 & Small Office & 3.5 & 43.4 & 12.5 & 1.8 & 41.1 & 22.6 & 0.32 & ----- \\
\hline 8 & Medium Office & 33.7 & 382.0 & 11.3 & 17.7 & 362.1 & 20.4 & 0.50 & ----- \\
\hline 9 & Large Office & 410.0 & 4250.0 & 10.4 & 215.9 & 4028.2 & 18.7 & 0.69 & ------ \\
\hline 10 & Fast-Food Restaurant $^{3}$ & 10.0 & 45.8 & 4.6 & 5.2 & 43.4 & 8.3 & 0.76 & ----- \\
\hline 11 & Sit-Down Restaurant $^{4}$ & 17.7 & 98.0 & 5.6 & 9.3 & 92.9 & 10.0 & 0.79 & ------ \\
\hline 12 & Stand-Alone Retail & 26.5 & 245.4 & 9.3 & 14.0 & 232.6 & 16.7 & 0.63 & ------ \\
\hline 13 & Strip Mall Retail & 35.4 & 187.2 & 5.3 & 18.6 & 177.4 & 9.5 & 0.40 & ------ \\
\hline 14 & Primary School & 63.5 & 718.0 & 11.3 & 33.4 & 680.5 & 20.4 & 0.61 & ----- \\
\hline 15 & Secondary School & 95.0 & 779.0 & 8.2 & 50.0 & 738.4 & 14.8 & 0.13 & 0.40 \\
\hline 16 & Warehouse & 7.1 & 5.7 & 0.8 & 3.7 & 5.4 & 1.4 & 0.21 & ------ \\
\hline
\end{tabular}

\footnotetext{
${ }^{3}$ This building type is also referred to as Quick-Service Restaurant.
}

${ }^{4}$ This building type is also referred to as Full-Service Restaurant. 
Five of the ventilation levels are intended for future review (see rows with an "Assumed VF" value). These values were either not extractable from this initial EnergyPlus analysis or the determined values were considered significantly different from intuitively expected levels. The RTUCC is not critically sensitive to the ventilation-fraction values. The load slope and load intercept are more critical.

\section{A.3 EnergyPlus OUTPut AND Post-Processing APPRoACH}

The hourly report (the CSV file produced by EnergyPlus) is post processed using R, a statistics analysis language. R scripts are run for each building to scan in the CSV file and analyze the hourly record.

\section{A.3.1 IDF (INPUT DATA FILES)}

In support of the ASHRAE Standard 90.1 Committee, PNNL developed a suite of 16 prototype building models in EnergyPlus. There are 17 different models for each of the building types. Each model complies with the prescriptive requirements of Standard 90.1, 2004 in each of the 17 DOE climate zones.

The 2004 versions of the IDF files, representing various building types, were obtained and used in this analysis.

The following is the filename of an example IDF file used in the analysis:

ASHRAE30pct_OfficeMedium STD2004_Chicago.idf

(Note: the restaurant IDF file was unintentionally a 2010 version.)

\section{A.3.2 OUTPUT VARIABLES}

The following variables are among those recorded in the CSV output files and were the basis for much of the post-processing analysis:

\section{Loads}

DXCOIL.DX.Coil.Sensible.Cooling.Energy.J..Hourly. (DX only, gross sensible cooling by system)

DXCOIL.DX.Coil.Total.Cooling.Energy.J..Hourly. (DX only, gross total (=S+L) cooling) Air.Loop.Total.Cooling.Coil.Energy.J..Hourly. (Includes both DX and chiller, gross total $(=\mathrm{S}+\mathrm{L})$ cooling)

Zone.Sys.Sensible.Cooling.Energy..J..Hourly. (Net sensible cooling, after economizer and reheat, delivered to zones. Includes sensible cooling delivered to zone when coils are off, such as economizer.)

Air.Loop.Total.Heating.Coil.Energy.J..Hourly. (Heating done by system. Includes reheat.) 


\section{Ventilation}

Zone.Mechanical.Ventilation.Mass.Flow.Rate..kg.s..Hourly.

Zone.Mechanical.Ventilation.Volume.Flow.Rate.Current.Density..m3.s..Hourly.

Zone.Mechanical.Ventilation.Cooling.Load.Increase..J..Hourly.

\section{Indoor Air}

Zone.Mean.Air.Temperature..C..Hourly.

Zone.Mean.Air.Humidity.Ratio....Hourly.

\section{Outdoor Air}

Environment.Outdoor.Dry.Bulb..C..Hourly.

Environment.Outdoor.Wet.Bulb..C..Hourly.

Environment.Outdoor.Barometric.Pressure..Pa..Hourly.

Environment.Outdoor.Air.Density..kg.m3..Hourly.

\section{Electricity Consumption}

Air Loop DX Cooling Coil Electric Consumption (compressor and condenser fan)

Air Loop Fan Electric Consumption (fans: not sure if this is all fans or just the evaporator)

\section{A.3.3 Post Processing IN R}

The two plots in Figure A-2 contrast the two sensible-load report variables. In the left plot, DXCOIL.DX.Coil.Sensible is an aggregate (sum) of all the air-loop (system) sensible coil loads (energy removed by coils). The right, Zone.Sys.Sensible, is an aggregate of all the sensible cooling delivered to the zones. The zone result (right) includes all sensible cooling delivered to the zone, even when the compressor is off. There is likely economizer cooling (compressor off) in the zone aggregate. 

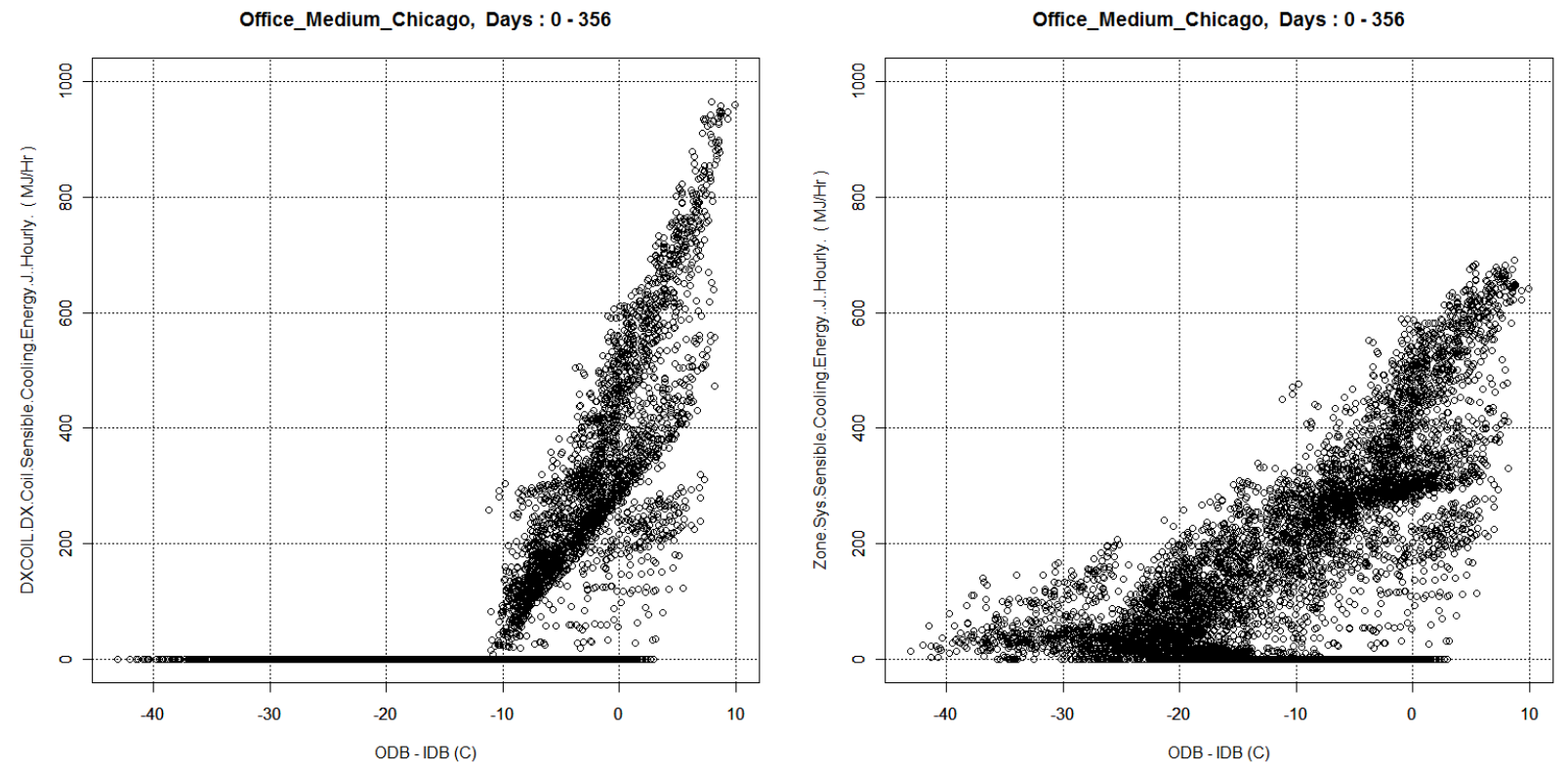

Figure A-2 Sensible cooling loads as affected by envelope temperature differential. Left: DX coil loads. Right: Aggregate of zone loads.

It is difficult to determine with certainty if these loads are net after the reheat. However, descriptions in the EnergyPlus Input Output reference lead the reader to assume the zone report (right plot) is a net effect (net cooling delivered to the zone, after reheat and economizing). The air-loop report (left plot) is strictly the output of the cooling coil (gross) needed to satisfy the thermostatic control. The air-loop cooling is the gross coil cooling needed to satisfy all sensible loads, including reheat. The two plots above support this interpretation; the right plot shows lower peak values in hot weather (because they are net) and more cooling in cool weather (because of economizing).

Annual sums for two plots in Figure A-2: Coil Loads $=9.13654 \mathrm{e}+11(\mathrm{~J}), \quad$ Zone Cooling $=$ $1.208048 \mathrm{e}+12(\mathrm{~J})$

The air-loop (or system) variable best reflects how hard the system has to work to satisfy all the loads. For this reason, this or related system variables will be the basis for developing response models.

In the analysis that follows, load variables are used as described here:

DXCOIL.DX.Coil.Sensible.Cooling.Energy is used whenever there is only DX cooling. Note that sensible coil load analysis is not possible in EnergyPlus if there is no DX cooling. System (Air.Loop) variables, such as Air.Loop.Total.Cooling.Coil, only have total $(=\mathrm{S}+\mathrm{L})$ versions.

DXCOIL.DX.Coil.Total.Cooling.Energy and Air.Loop.Total.Cooling.Coil.Energy are equivalent if there is only DX cooling. If there are both DX and chiller, the DXCOIL.DX.Coil.Total will provide the DX portion of the total $(=\mathrm{S}+\mathrm{L})$ cooling. If there are 
both DX and chiller, the Air.Loop.Total variable will provide a sum of the DX and chiller coil cooling.

In the R modeling of the loads, a "bottom scraping" approach is used to subset the hourly data. This removes zero-value or near-zero value loads before regression is done. (Zero-level loads are clearly visible in the raw load plot on the previous page.) The idea is that zeros should not contribute to the regression; the model should reflect the loads presented to and processed by the system. Including zeros in the regressions inappropriately lowers the predictions of the load model. The default scraping mode (GEZ), is used to Exclude Zero-value loads and any load data occurring at temperatures lower than the lowest Greater-than-Zero load. An alternate approach $(\mathbf{G M})$ is used on some buildings that produce a large number of near-zero loads. The GM approach accepts all data that is Greater than a minimum value as established as a fraction of the Maximum hourly load. In this approach, the tolerance for ground clutter (near-zero values) can be varied to see at what level their effect stops. The lowest tolerance that eliminates the effect is used.

Loads are aggregated (summed) with similar systems or zones. For example, the Medium Office building type has three DX systems supporting three air loops. These are summed to produce an aggregate DX load.

Indoor temperature is calculated as an average of the zone temperatures. These are not weighted by zone size and use only a simple average. For some buildings, unconditioned zones may be excluded from the average. For example, the Fast-Food Restaurant ${ }^{5}$ building type has an unconditioned attic zone that basically floats at the outdoor temperature; this is excluded from the temperature average.

Ventilation loads are calculated (in R post processing) for any zone reporting mechanical ventilation. Standard ASHRAE algorithms are used to calculate sensible and total ventilation loads. The calculation uses indoor and outdoor psychrometric conditions and reported hourly airmass flows. Ventilation loads are calculated at the zone level and then aggregated to produce a building sum.

The option of producing daily average loads was considered. Figure A-3 shows daily average loads for the DXCOIL.DX plot in Figure A-2. This plot includes the zero-value points in the daily average; this inclusion reduces the slope. This approach was not used because the hourly approach offered more control over excluding the near-zero value points. Also, with the hourly approach, it is clearer to the reader which points have been excluded.

\footnotetext{
${ }^{5}$ This building type is also referred to as Quick Service Restaurant.
} 


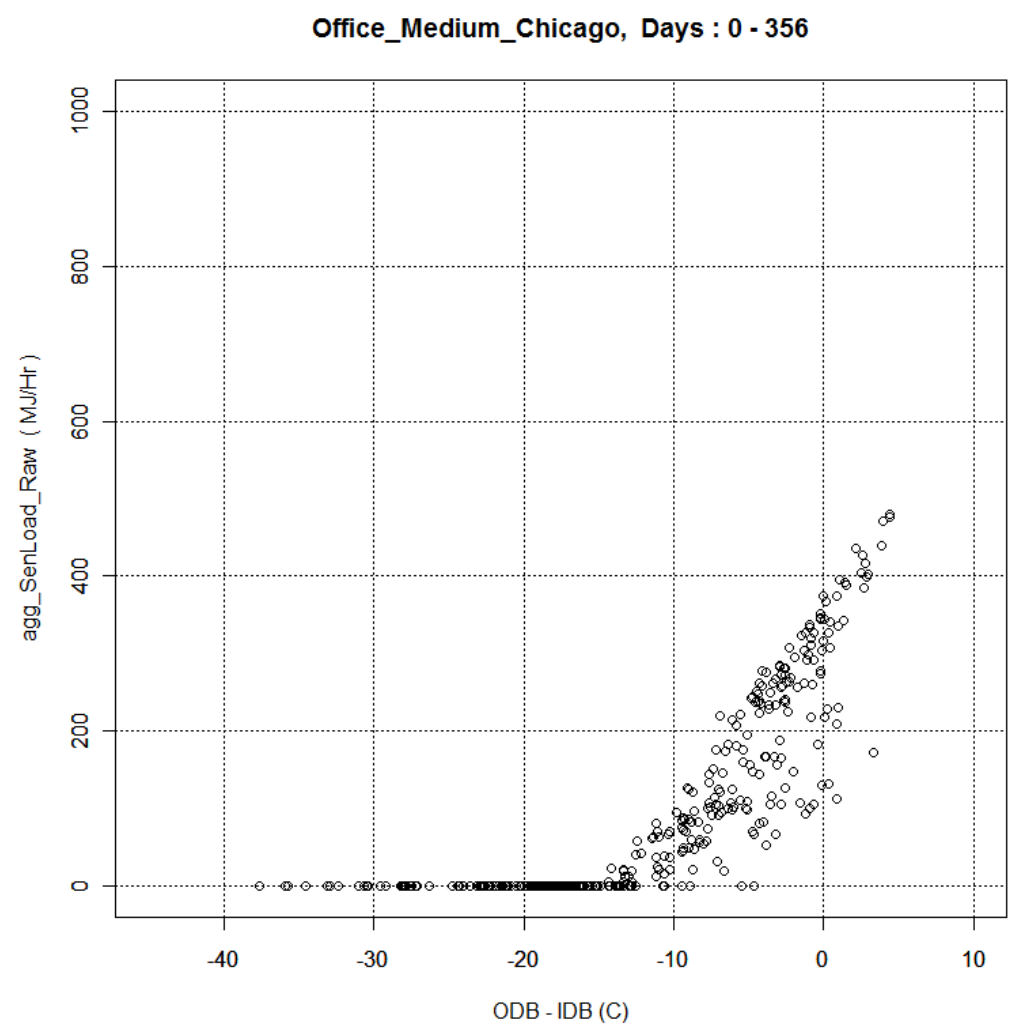

Figure A-3 Daily average sensible cooling loads for Chicago Medium Office as affected by envelope temperature differential.

\section{A.4 Sensitivity Testing}

The following sections have post-processing results from the EnergyPlus runs that are in support of the findings and sensitivity analysis. Section A.4.1 has the most detailed explanations and serves as a key for interpreting the results for each building type as reported in Section A.5.

\section{A.4.1 Chicago Fast-Food Restaurant}

The post-processing analysis output for this first example run is explained here in detail. Most of the following runs will show a similar output. Differences are noted.

\section{A.4.1.1 Sensible Load}

In the plots of Figure A-4 the sum of the sensible-coil loads is shown left and the sum of the sensible-ventilation loads is shown right. Both plots show how the loads respond to the outdoorindoor temperature differential. Both plots show a linear-model line in red. The plot title identifies the building type and any special adaptations done to the IDF input file. The title also identifies the range of days used in the plot. 

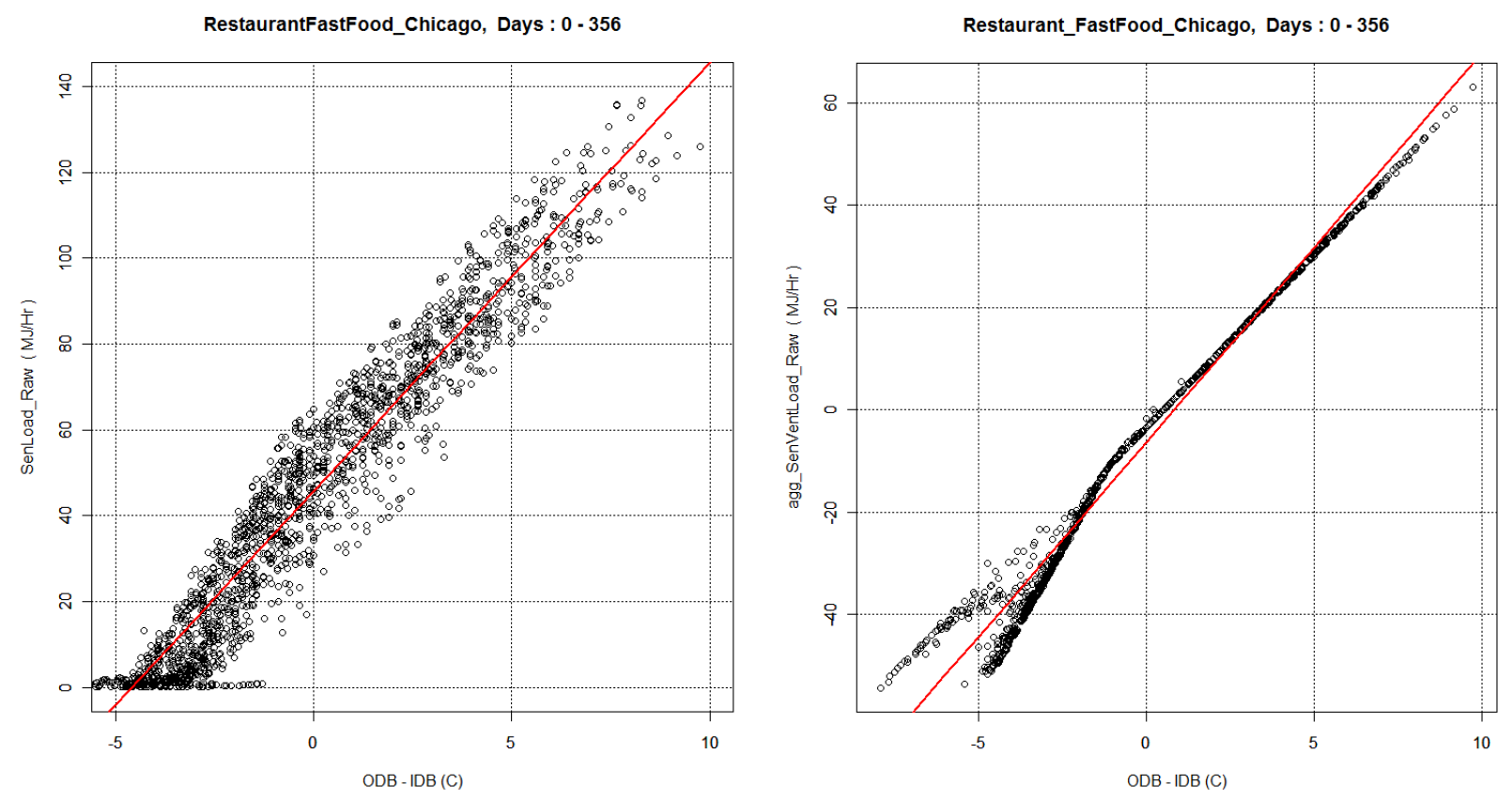

Figure A-4 Raw sensible loads for Chicago Fast-Food Restaurant as affected by envelope temperature differential. Left: sensible load (Intercept $=45.8+/-0.2 \mathrm{MJ} / \mathrm{h}$; Slope $=9.97+/-0.07$ $\mathrm{MJ} / \mathrm{hC})$. Right: sensible ventilation load $($ Slope $=7.61+/-0.03$; Slope Fraction $=7.61 / 9.97=$ $0.76)$.

Slope and intercept are reported in the figure caption for the response model for coil loads (left plot). Only slope is reported for the ventilation model (right plot). Generally ventilation models have a zero or a nearly zero intercept. The slope and intercept form the main part of coil-load response model. This is then used to calculate the S\&I fraction, as defined in Equation 1 in the introduction. In some cases this result is shown for three example cities (see Table A.2). For this case, the fraction of response caused by ventilation is $7.61 / 9.97=0.763$. This is shown in the figure caption after the ventilation slope. This slope fraction is the third parameter needed for the building response signature.

Table A.2 Calculation of S\&I fraction in three cities using the Fast-Food building model.

\begin{tabular}{c|c|c} 
City & DT & $\begin{array}{c}\text { S\&I } \\
\text { Fraction }\end{array}$ \\
\hline SanFran & 0.8 & 0.86 \\
\hline Chicago & 7.5 & 0.38 \\
\hline Phoenix & 18.0 & 0.20
\end{tabular}




\section{A.4.1.2 Sensible Ventilation Load}

The left plot in Figure A-5 shows the aggregate ventilation load for both kitchen and dining zones. The inside temperature is a simple average of the two zone temperatures. The raw plot on the right also includes loads less than or equal to zero. This is shown here for comparison with the following two individual-zone plots (see Figure A-6 and Figure A-7). This demonstrates that the model's slope, when based on aggregate zone data, is equal to the sum of the two individual zone-model slopes $(1.8+5.0=6.8)$. The right-side plot shows the raw, unfiltered, load data.
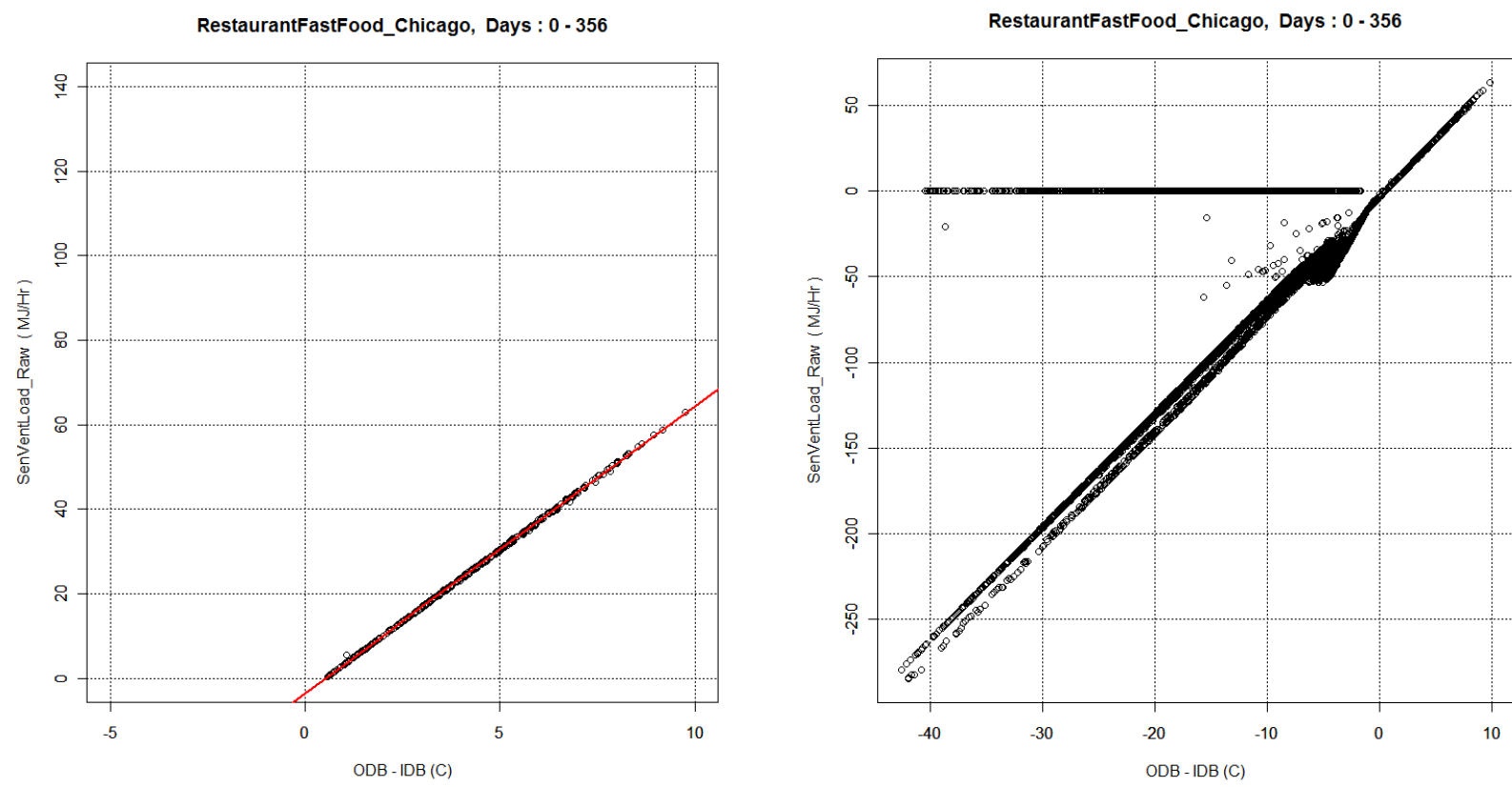

Figure A-5 Sensible ventilation load for Kitchen and Dining zones as affected by envelope temperature differential. Left: filtered to exclude negative loads; Slope $=6.8$. Right: raw data. 
Figure A-6 and Figure A-7 show single-zone ventilation loads. These plots demonstrate that the sum of the two zone slopes is equal to the slope in the aggregate plot $(1.8+5.0=6.8)$.

s.
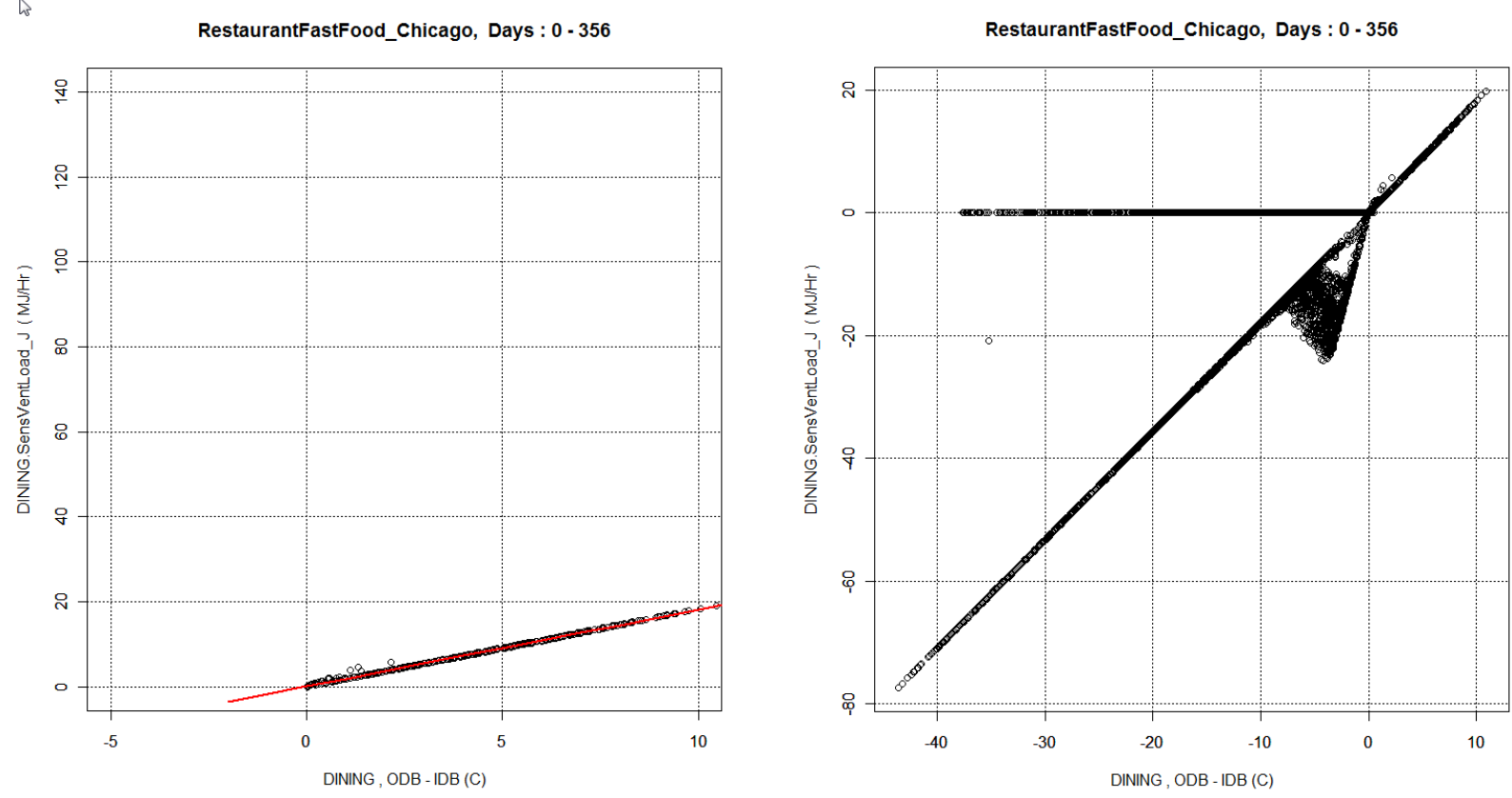

Figure A-6 Sensible ventilation load for Dining zone only. Left: filtered to exclude negative loads; Slope $=1.8$. Right: raw data.
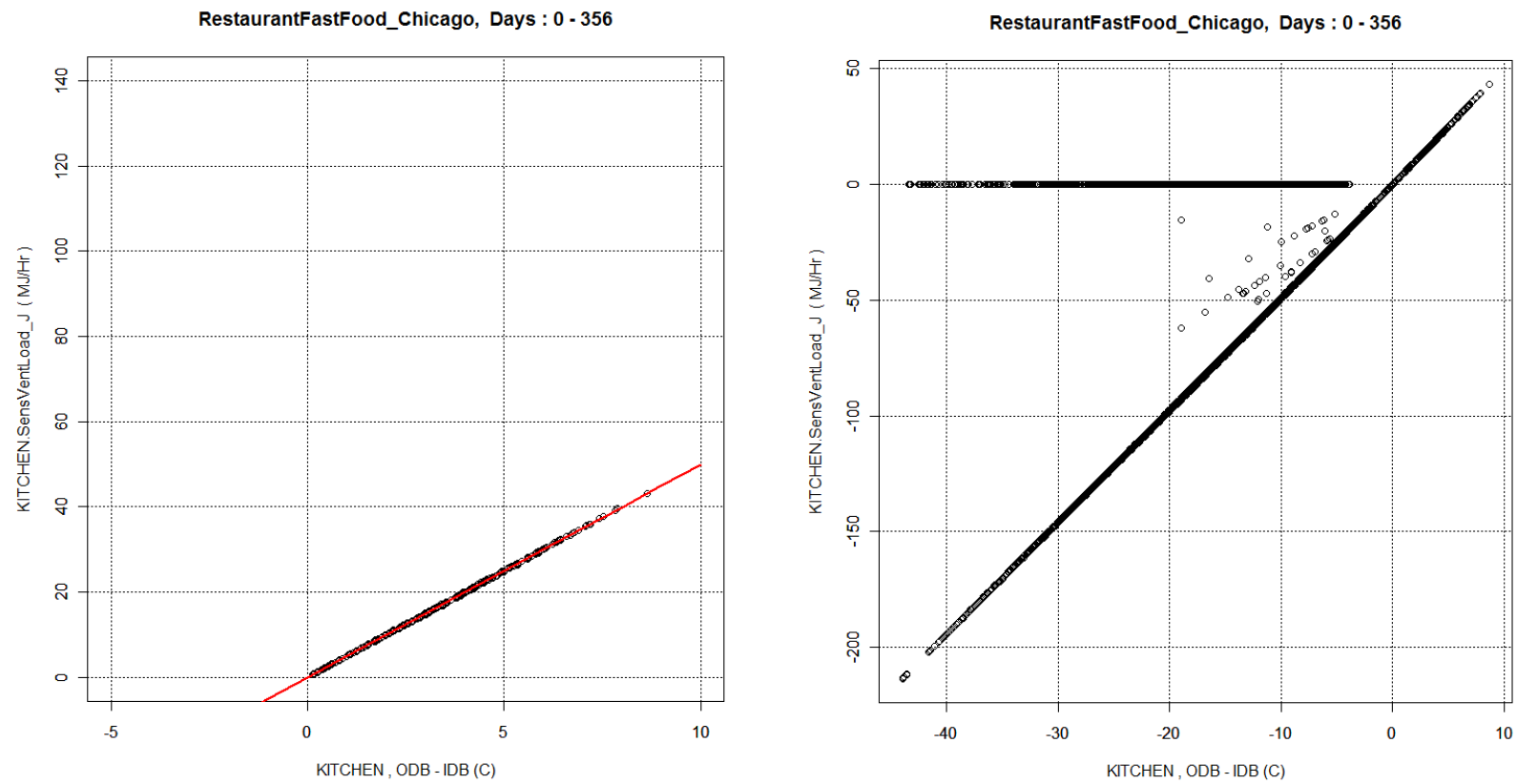

Figure A-7 Sensible ventilation load for Kitchen zone only. Left: filtered to exclude negative loads; Slope $=5.0$. Right: raw data. 


\section{A.4.1.3 Total (=sensible+latent) Loads}

The total (sensible + latent) load data in Figure A-8 illustrates how the response model from total loads produces S\&I fraction calculations in Table A.3 that are very similar to those in Table A.2 which result from the sensible data shown in Figure A-4. This result also demonstrates that the percentage of the ventilation line is similar to that in the sensible analysis. This comparison justifies using the total load data for those buildings that do not have DX cooling systems.
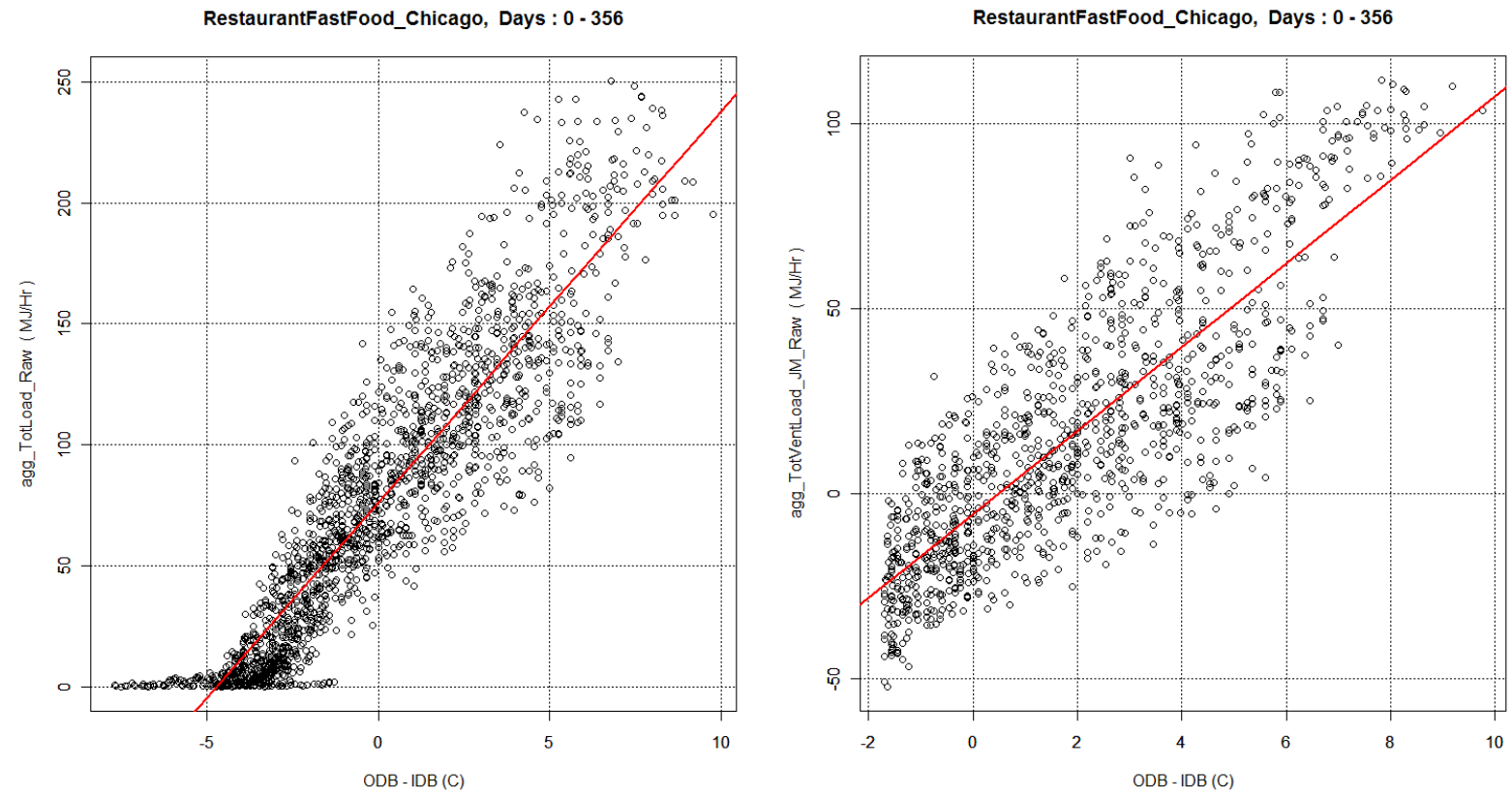

Figure A-8 Raw total (latent + sensible) load for Chicago Fast-Food Restaurant as affected by envelope temperature differential. Left: total load $($ Intercept $=76.28 \mathrm{MJ} / \mathrm{h}$; Slope $=16.16$ $\mathrm{MJ} / \mathrm{hC})$. Right: total ventilation load $($ Slope $=11.31$; Slope Fraction $=11.31 / 16.16=0.70)$.

Table A.3 Calculation of S\&I fraction in three cities using the total-load version of the FastFood Restaurant building model.

\begin{tabular}{c|c|c} 
City & DT & $\begin{array}{r}\text { S\&I } \\
\text { Fraction }\end{array}$ \\
\hline SanFran & 0.8 & 0.86 \\
\hline Chicago & 7.5 & 0.39 \\
\hline Phoenix & 18.0 & 0.21
\end{tabular}




\section{A.4.2 Chicago Medium Office / Response Models and Weather Data}

This section is a summary of the tests used to determine if the resulting response models are affected by the weather data (city) used in the simulation runs. Table A.4 shows that the ratio of the intercept to the slope is always within $5 \%$ of the baseline case (Chicago weather with the Chicago Medium Office building). The consistency in this ratio is a direct indicator of the similarity of these models and how they will behave when scaled to match capacity in the RTUCC. This expected similarity in the calculator's output is a result of the scaling operation, which is applied equally to both the slope and the intercept of the linear model. In the subsections that follow, detailed results and data plots are presented that yield each of the rows in Table A.4.

Table A.4 Summary of Chicago Medium Office response model as affected by the weather data of four cities.

\begin{tabular}{r|c|c|c}
$\begin{array}{r}\text { Weather } \\
\text { City }\end{array}$ & $\begin{array}{c}\text { Intercept } \\
(\mathrm{MJ} / \mathrm{hC})\end{array}$ & $\begin{array}{c}\text { Slope } \\
(\mathrm{MJ} / \mathrm{hC})\end{array}$ & $\begin{array}{c}\text { Intercept/slope } \\
(\mathrm{C})\end{array}$ \\
\hline Chicago & 382 & 33.7 & 11.3 \\
\hline Phoenix & 317 & 27.1 & 11.7 \\
\hline San Francisco & 404 & 34.1 & 11.8 \\
\hline Miami & 327 & 37.7 & 11.8
\end{tabular}

The following subsections also use their weather-specific version of the model to calculate the S\&I fraction for several cities. Again, the S\&I fraction is the ratio of internal load to the capacity of the unit at design conditions. It is to be expected that this calculated ratio for a particular city should be similar in each of these subsections, and that is the case. For example, the four different weather versions of the model yield S\&I values of 0.39, 0.39, 0.40, and 0.33 when used in calculating the S\&I ratio for Phoenix.

In summary, this test supports the idea that one linear response model (for each building type) can be applied across the country. Refinements could be made by developing multiple versions of each building model to better represent secondary climate/building interactions. But the gain offered in this type of refinement appears to be small relative to general expectations for the uncertainty of the calculator. 


\section{A.4.2.1 Chicago Weather}

Figure A-9 and Table A.5 illustrate the baseline case: the Chicago version of the Medium Office building run with Chicago weather. This case will be compared to others where the Chicago Medium Office building is run with different weather data. It will also be compared to a different city version of the Medium Office building type run with the corresponding weather data.
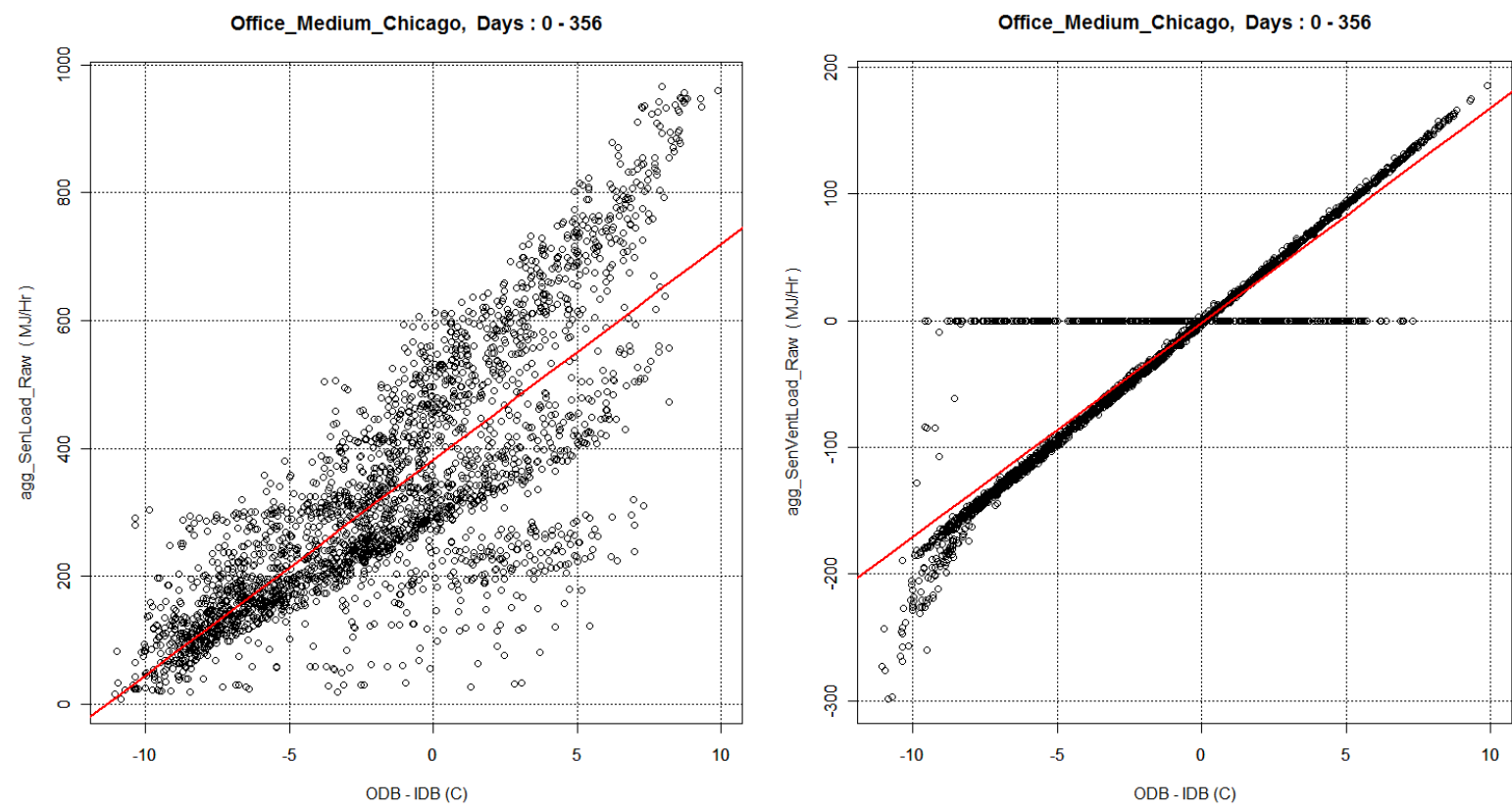

Figure A-9 Chicago Medium Office with Chicago weather. Raw sensible load as affected by envelope temperature differential. Left: sensible load (Intercept $=382+/-2 \mathrm{MJ} / \mathrm{h}$; Slope $=33.7$ +/- 0.5 MJ/hC). Right: sensible ventilation load (Slope $=16.91+/-0.13$; Slope Fraction $=0.50$ ).

Table A.5 Calculation of S\&I fraction in three cities using a Chicago Medium Office building model with Chicago weather.

\begin{tabular}{r|c|c} 
City & DT & $\begin{array}{c}\text { S\&I } \\
\text { Fraction }\end{array}$ \\
\hline SanFran & 0.8 & 0.94 \\
\hline Chicago & 7.5 & 0.60 \\
\hline Phoenix & 18.0 & 0.39
\end{tabular}




\section{A.4.2.1.1 Simple Outdoor Temperature Plot}

The plot in Figure A-10 for the Chicago Medium Office building type uses the raw outdoor drybulb (ODB) temperature, not the $\Delta T$ that is based on the aggregate zone temperature, as is used in previous plots. Correlation levels are similar; some parts of the data appear more tightly correlated. The $\Delta T$ plot offers independence from thermostat setpoint and setback.

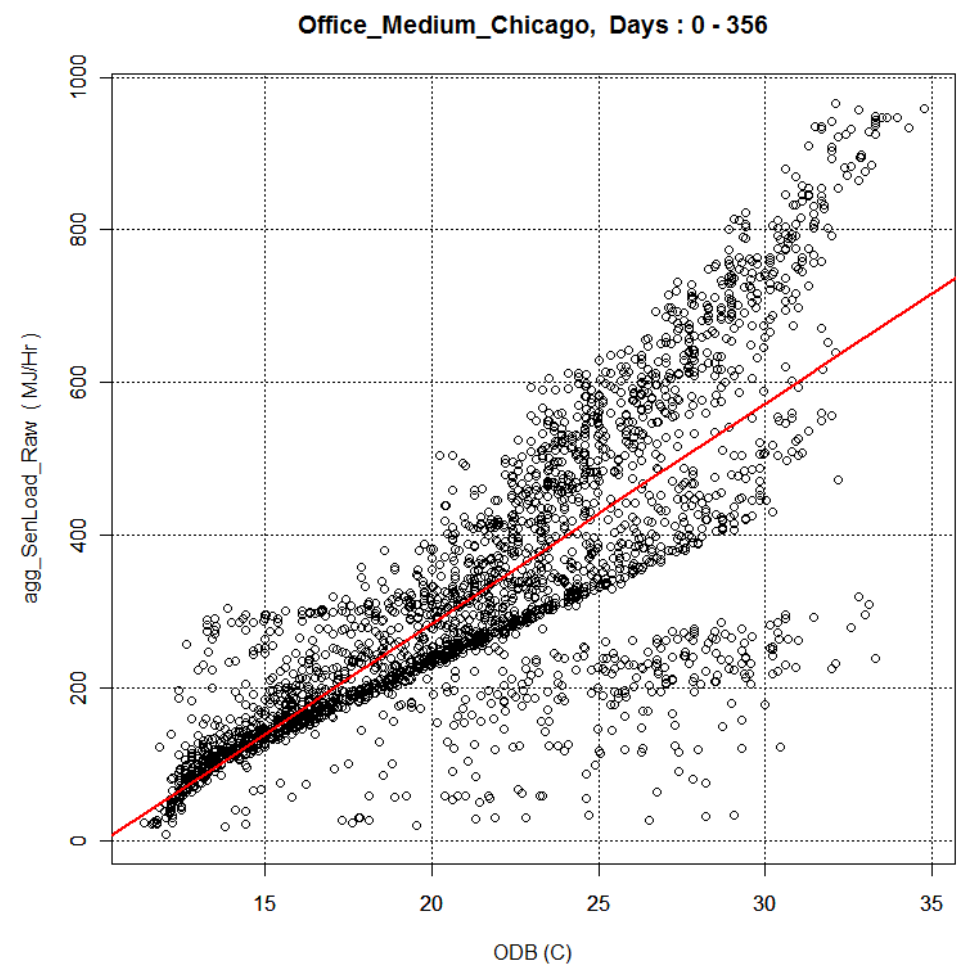

Figure A-10 Raw sensible load for the Chicago Medium Office building type. Load response shown as affected by outdoor temperature $(T$, not $\Delta T)$. 


\section{A.4.2.1.2 Setback Turned Off}

The zone-aggregated temperature plots in Figure A-11 illustrate the effect of turning off setback in the IDF file. The left plot has setback turned off and has a higher winter setpoint. The right plot uses setpoints and setback and corresponds to the base case Chicago run. The plots in Figure A-12 show the impact on the cooling-load response model caused by removing setback.
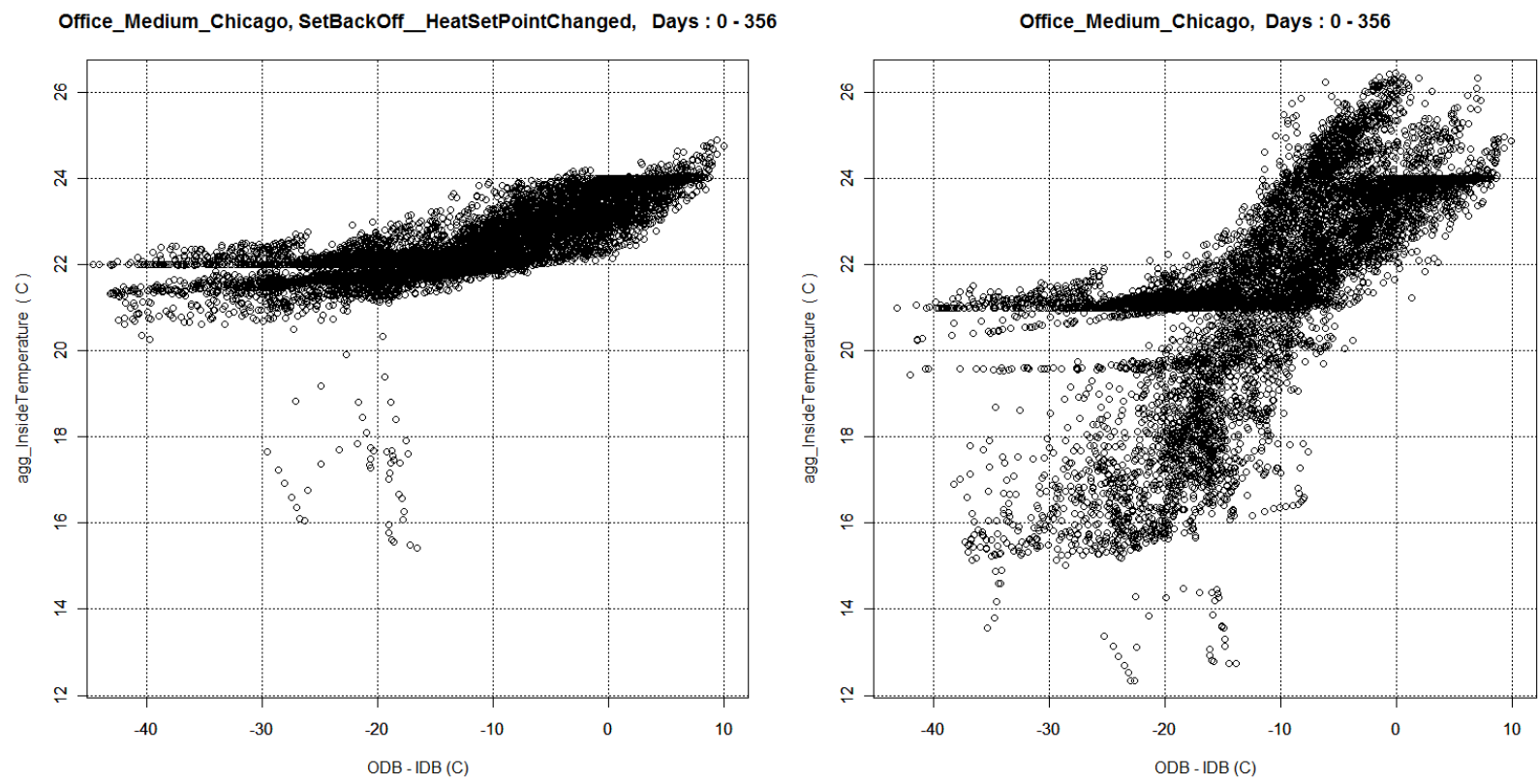

Figure A-11 Indoor temperature as correlated with temperature differential. Left: no setback. Right: setback enabled. 
For the data in Figure A-12 and Table A.6 the setback has been turned off. The effect of setback on the load model projections is small. Refer to Figure A-11 for a corresponding illustration using a temperature plot.
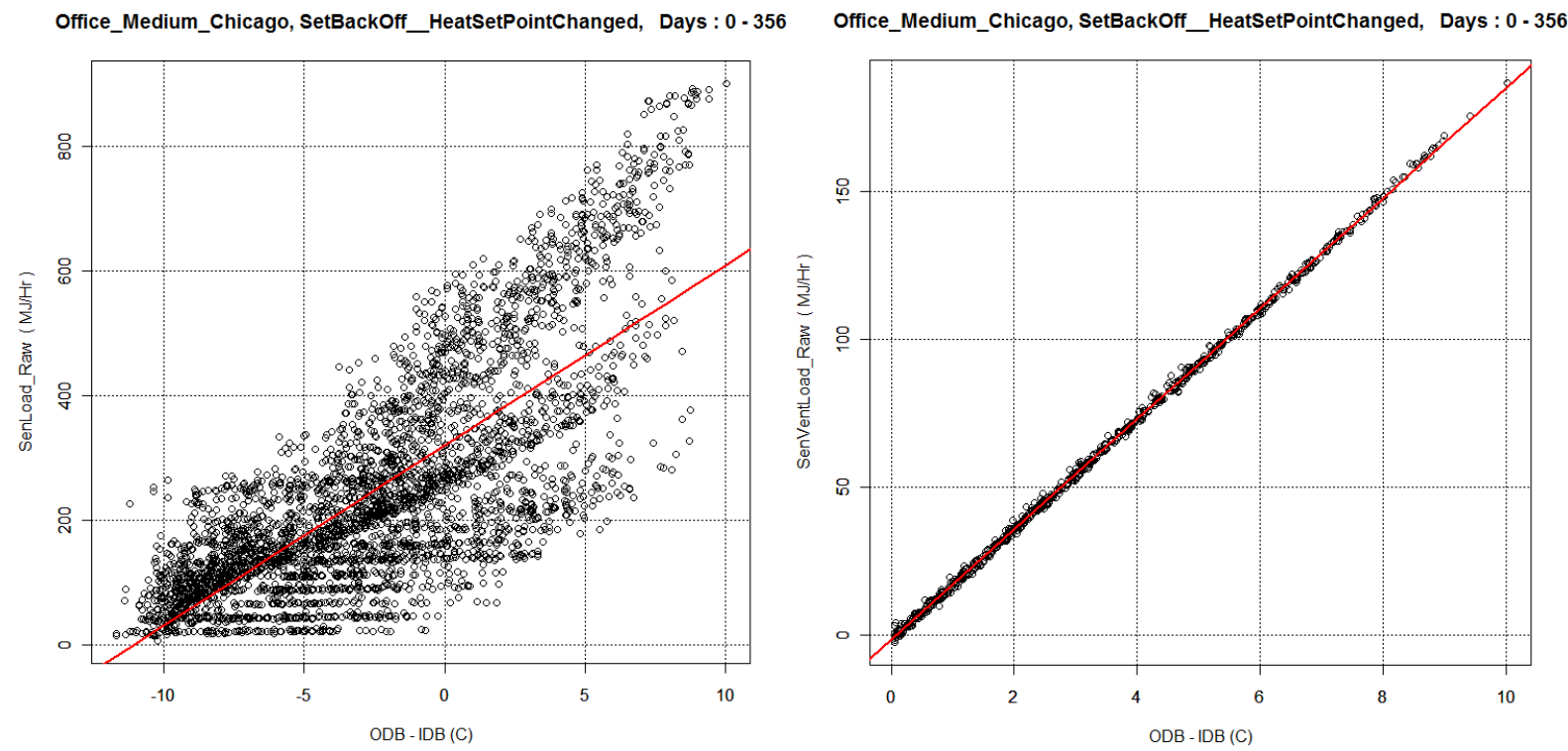

Figure A-12 Chicago Medium Office with no setback. Raw sensible load as affected by envelope temperature differential. Left: sensible load (Intercept $=320.4 \mathrm{MJ} / \mathrm{h}$; Slope $=28.87$ $\mathrm{MJ} / \mathrm{hC})$. Right: sensible ventilation load (Slope $=18.67$; Slope Fraction $=18.67 / 28.87=0.65)$.

Table A.6 Calculation of S\&I fraction in three cities using the Chicago Medium Office building model with no setback.

\begin{tabular}{c|c|c} 
City & DT & $\begin{array}{c}\text { S\&I } \\
\text { Fraction }\end{array}$ \\
\hline SanFran & 0.8 & 0.93 \\
\hline Chicago & 7.5 & 0.60 \\
\hline Phoenix & 18.0 & 0.38
\end{tabular}

\section{A.4.2.1.3 No Ventilation}

The plots in Figure A-13 illustrate the effect of turning off mechanical ventilation in the IDF file. The two plots on the right show the base case with ventilation on (bottom right shows daily on/off pattern). For the two plots on the left, the mechanical cooling ventilation has been effectively turned off. However, there appears to be some ventilation in the heating season. The slope of the red load-line (bottom left) is 19.2. 

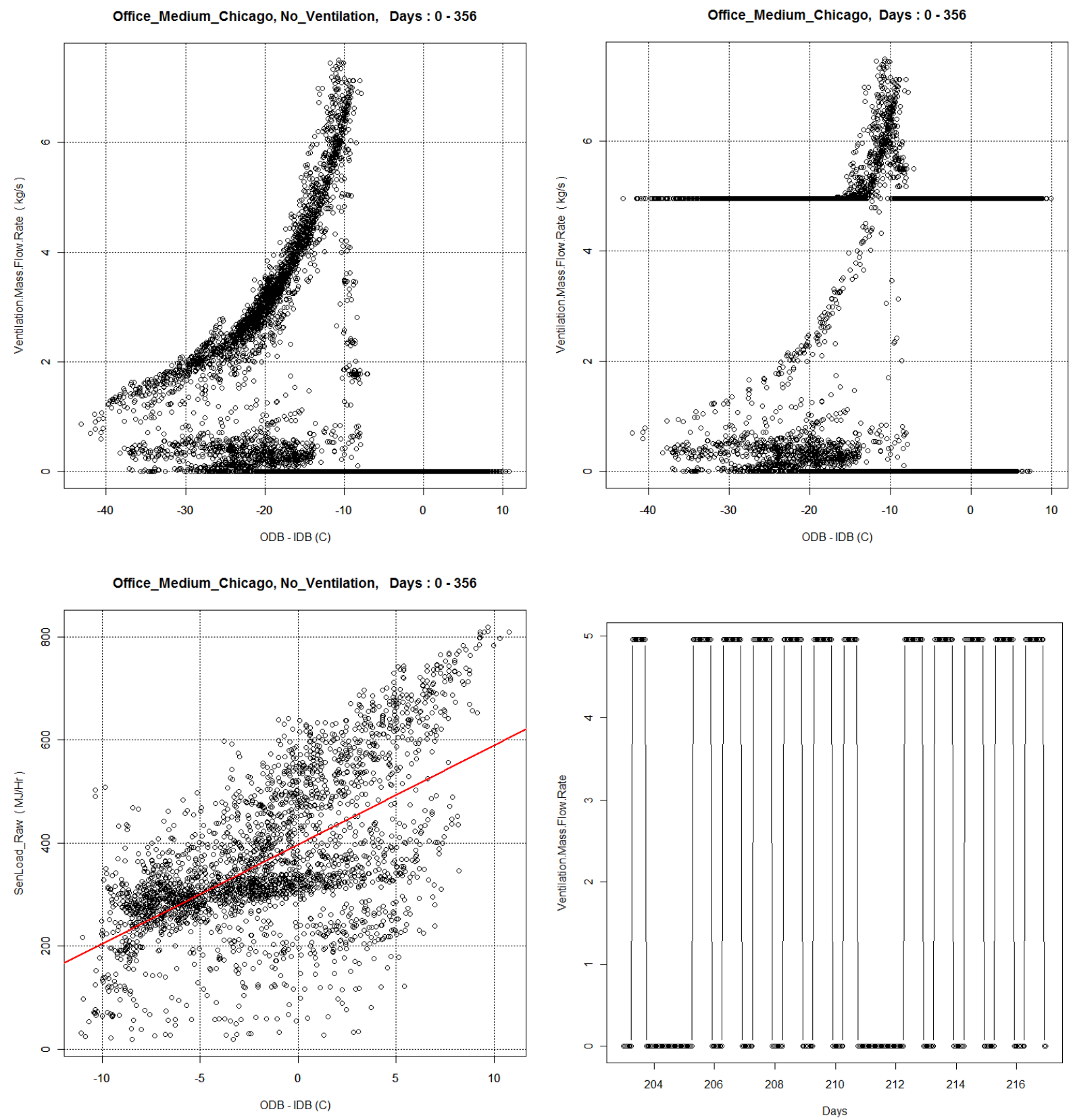

Figure A-13 Data plots illustrating the patterns and impact of mechanical ventilation. Left: no mechanical ventilation. Right: mechanical ventilation enabled.

The end result of controlling ventilation levels this way (in the IDF files) is similar to that when done by calculating ventilation loads in post processing. The slope of 19.2 for the red load-line in Figure A-13 is reasonably close to what would be predicted by subtracting the slope of the calculated ventilation load (18.7) from the slope of the total sensible load-line of the baseline case that includes ventilation (see Figure A-9: 33.7 - 18.7 = 15.0). 
The two plots in Figure A-14 are provided to show the building's heating and cooling loads on the same plot. The possibility illustrated here is that together the heating and cooling loads might give a cleaner overall picture of the building's response to outdoor temperature.
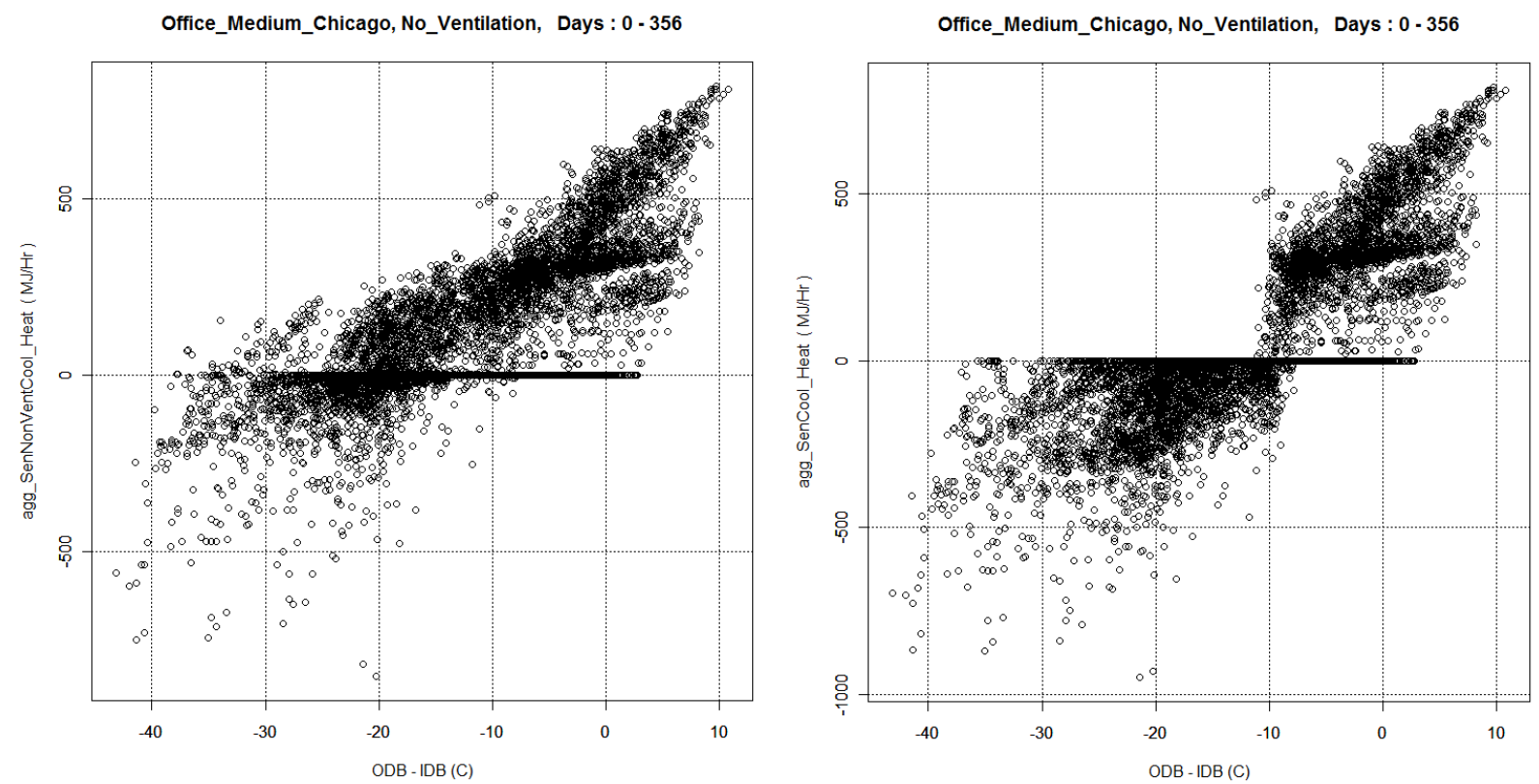

Figure A-14 Heating and cooling loads in response to changing $\Delta T$. Left: heating-ventilation load is removed. Right: raw data (includes heating-ventilation loads). Both plots have mechanical ventilation turned off.

Heating loads are assigned negative values and then are added to the time series of cooling loads. Both plots have the mechanical ventilation turned off in their IDF file. (Note: this shutdown left some mechanical ventilation in the heating season.)

The right side plot is raw data. The left side has the calculated heating ventilation subtracted. This tends to shift up heating load and produce a true non-ventilation load-line for both heating and cooling.

This was not conclusive as to whether cooling and heating load data would produce a better load model. The idea is presented here for possible future analysis. 


\section{A.4.2.2 Phoenix Weather}

The results in Figure A-15 illustrate the behavior of the Chicago Medium Office building with Phoenix weather. A significant part of the data is at higher temperatures (the Chicago data is mostly below a 10 degree differential). However, the S\&I calculations in Table A.7, where the model is based on Phoenix weather, are very similar to those based on Chicago weather (Table A.5).
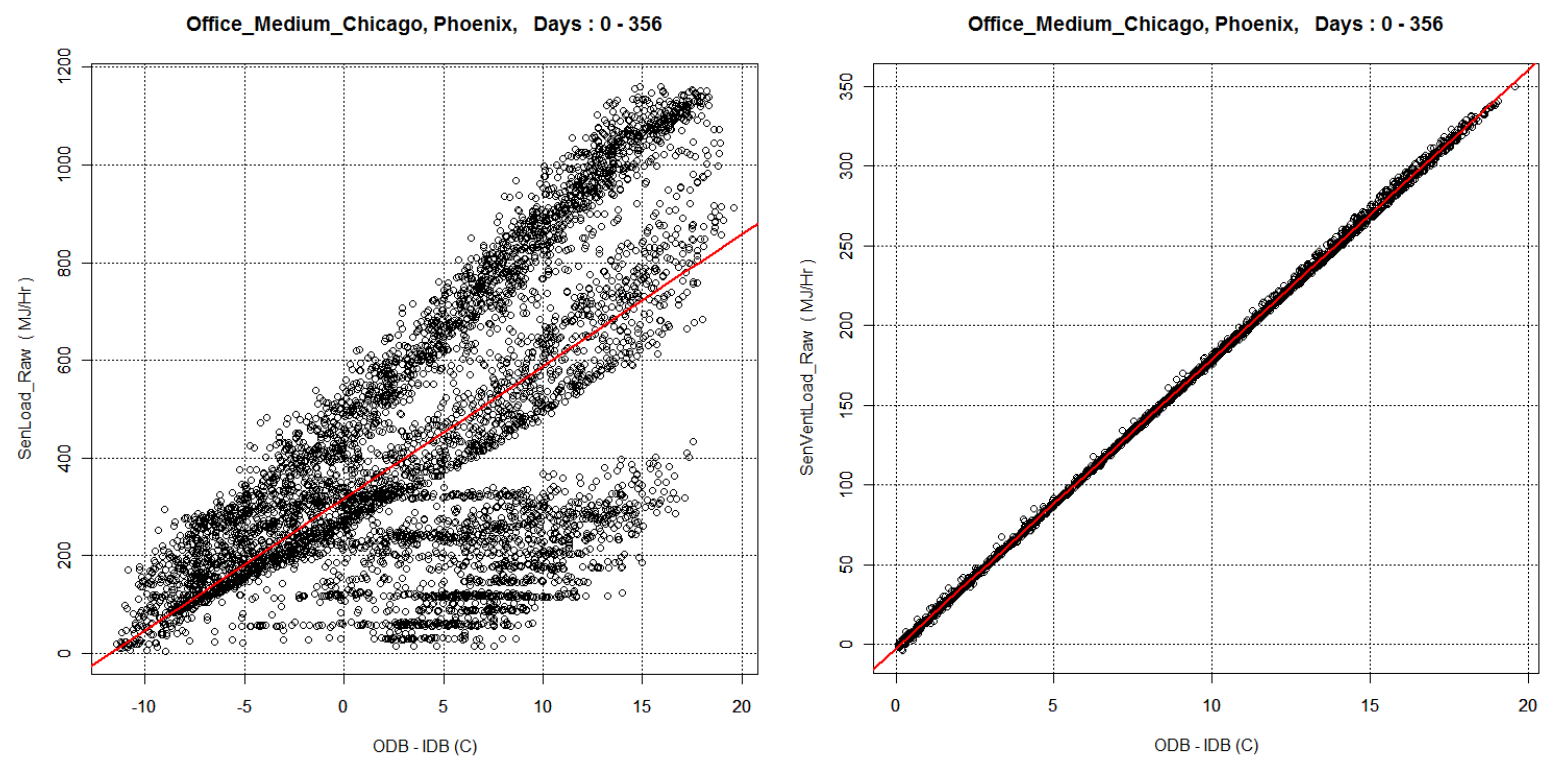

Figure A-15 Raw sensible loads for the Chicago Medium Office building simulated with Phoenix weather as affected by envelope temperature differential. Left: sensible load (Intercept $=317.1 \mathrm{MJ} / \mathrm{h}$; Slope = 27.06 MJ/hC). Right: sensible ventilation load (Slope = 18.13; Slope Fraction $=18.13 / 27.06=0.67)$.

Table A.7 Calculation of S\&I fraction in three cities using the linear model resulting from the Chicago Medium Office building with Phoenix weather.

\begin{tabular}{c|c|c} 
City & DT & $\begin{array}{c}\text { S\&I } \\
\text { Fraction }\end{array}$ \\
\hline SanFran & 0.8 & 0.94 \\
\hline Chicago & 7.5 & 0.61 \\
\hline Phoenix & 18.0 & 0.39
\end{tabular}




\section{A.4.2.3 San Francisco Weather}

This run (Figure A-16) simulates the Chicago Medium Office building with San Francisco weather. Note that most of the data is at outdoor temperatures below the setpoint. However, the S\&I results (Table A.8) are very similar to the corresponding results when simulated with Chicago weather (Table A.5).
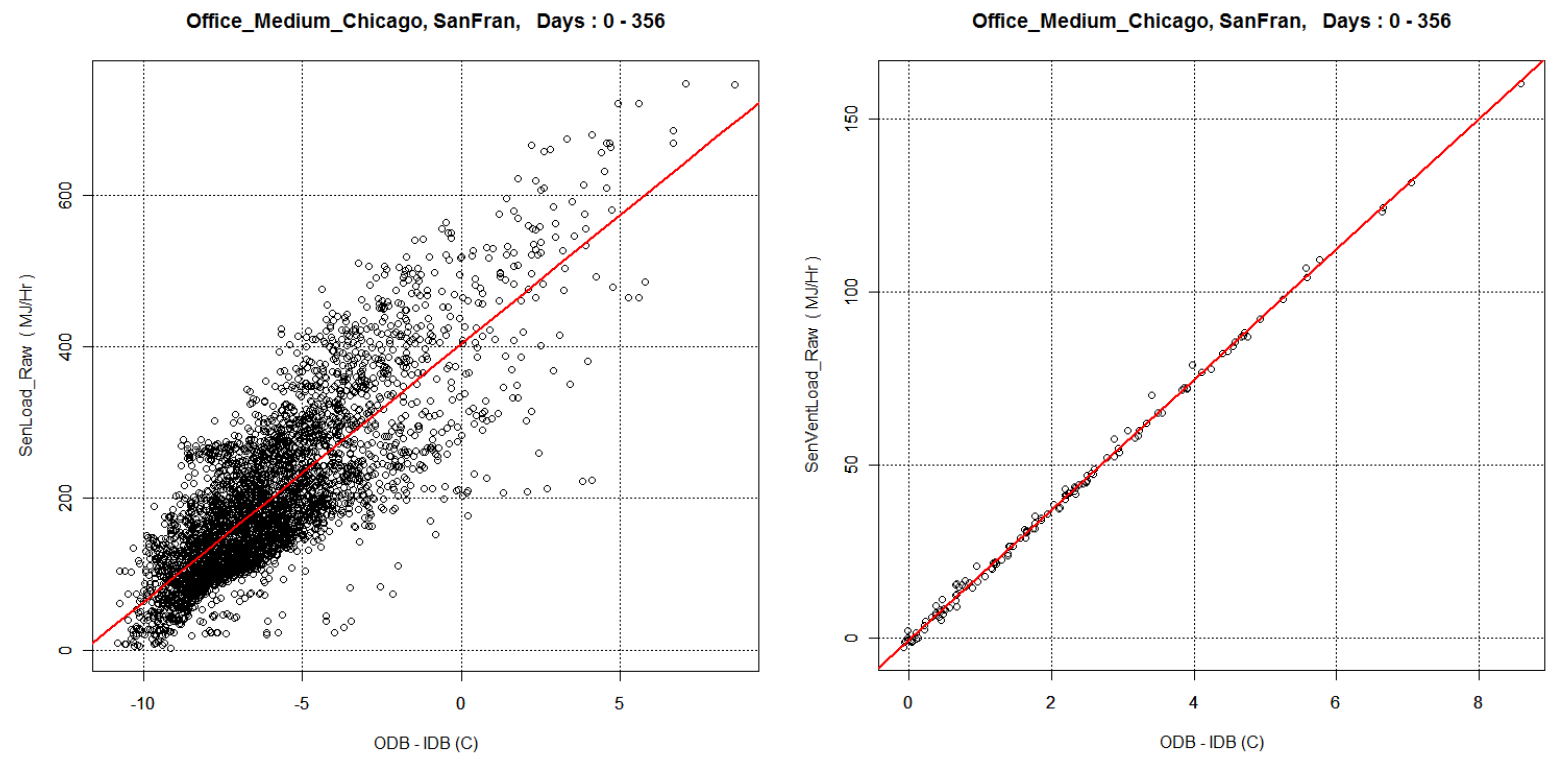

Figure A-16 Raw sensible loads for the Chicago Medium Office building simulated with San Francisco weather as affected by envelope temperature differential. Left: sensible load $($ Intercept $=403.9 \mathrm{MJ} / \mathrm{h}$; Slope $=34.05 \mathrm{MJ} / \mathrm{hC})$. Right: sensible ventilation load $($ Slope $=18.84$; Slope Fraction $=18.84 / 34.05=0.55$ ).

Table A.8 Calculation of S\&I fraction in three cities using the linear model resulting from the Chicago Medium Office building with San Francisco weather.

\begin{tabular}{c|c|c} 
City & DT & $\begin{array}{c}\text { S\&I } \\
\text { Fraction }\end{array}$ \\
\hline SanFran & 0.8 & 0.94 \\
\hline Chicago & 7.5 & 0.61 \\
\hline Phoenix & 18.0 & 0.40
\end{tabular}




\section{A.4.2.4 Miami Weather}

This run (Figure A-17) simulates the Chicago Medium Office building with Miami weather. Notice that most of the data is at outdoor temperatures cooler than Phoenix, a similar overall range to Chicago, but with much more of the data at deltas above setpoint $(\Delta T=0)$. However, the S\&I results (Table A.9) are lower but still reasonably similar to those calculated from a model based on Chicago weather (Table A.5).
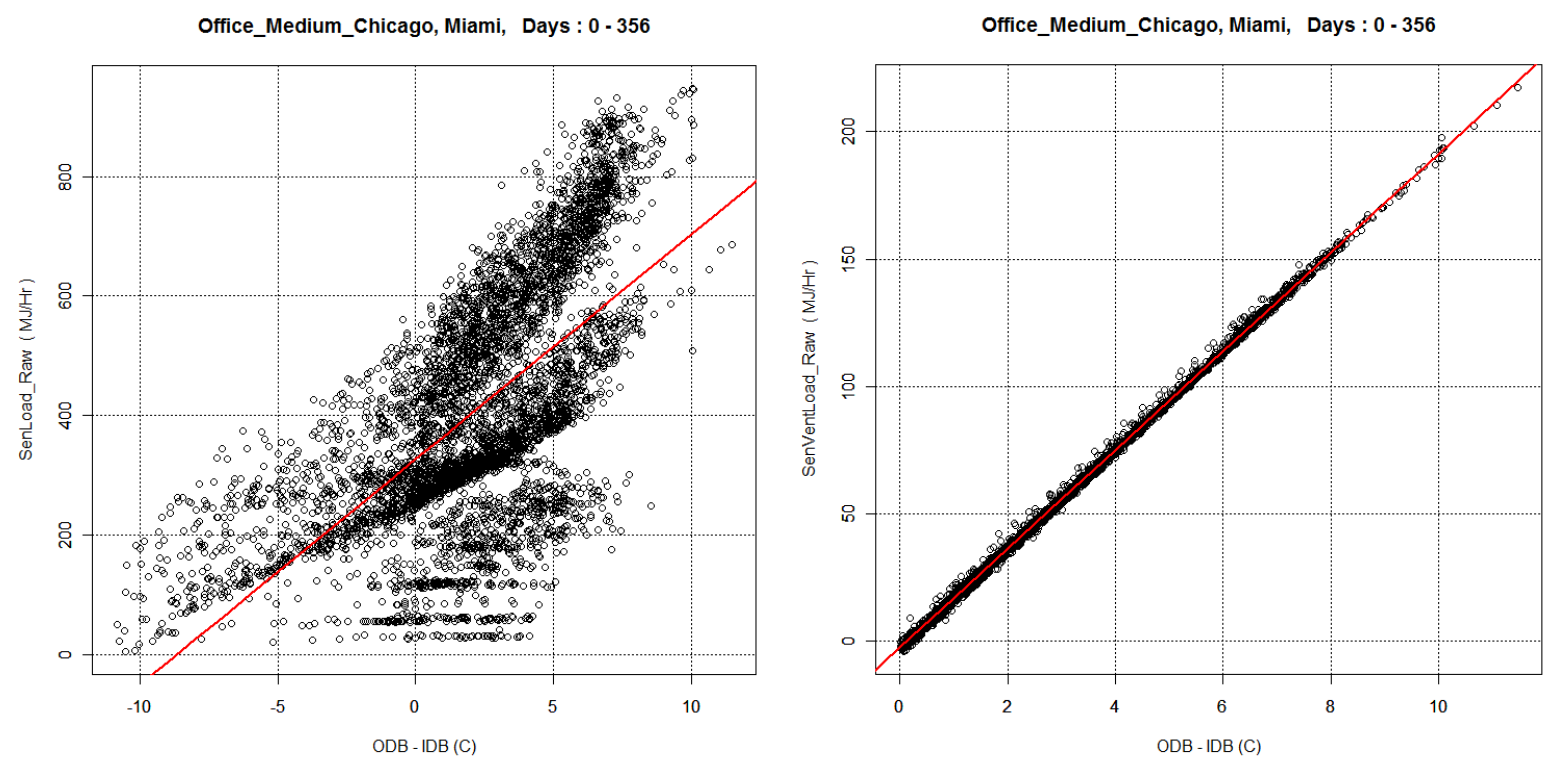

Figure A-17 Chicago Medium Office building type with Miami Weather. Raw sensible load as affected by envelope temperature differential. Left: sensible load (Intercept $=326.55 \mathrm{MJ} / \mathrm{h}$; Slope $=37.66 \mathrm{MJ} / \mathrm{hC})$. Right: sensible ventilation load (Slope = 19.38; Slope Fraction $=$ $19.38 / 37.66=0.51)$.

Table A.9 Calculation of S\&I fraction in three cities using the Chicago Medium Office building model with Miami weather.

\begin{tabular}{c|c|c} 
City & DT & $\begin{array}{c}\text { S\&I } \\
\text { Fraction }\end{array}$ \\
\hline SanFran & 0.8 & 0.92 \\
\hline Chicago & 7.5 & 0.54 \\
\hline Phoenix & 18.0 & 0.33
\end{tabular}




\section{A.4.3 Phoenix Medium Office}

This run result (see Figure A-18 and Table A.10) is for the Phoenix Medium Office (the Phoenix version) of the Medium Office building type simulated with Phoenix weather. The intercept to slope ratio of $327.65 / 29.45=11.1$ for this run is within $2 \%$ of the Chicago version run with Chicago weather (shown in Table A.4). Also notice the S\&I results here in Table A.10 are very similar to the Chicago-Chicago result (also shown in Table A.4).

This result is supporting evidence for using only one version of the IDF files to represent a building.
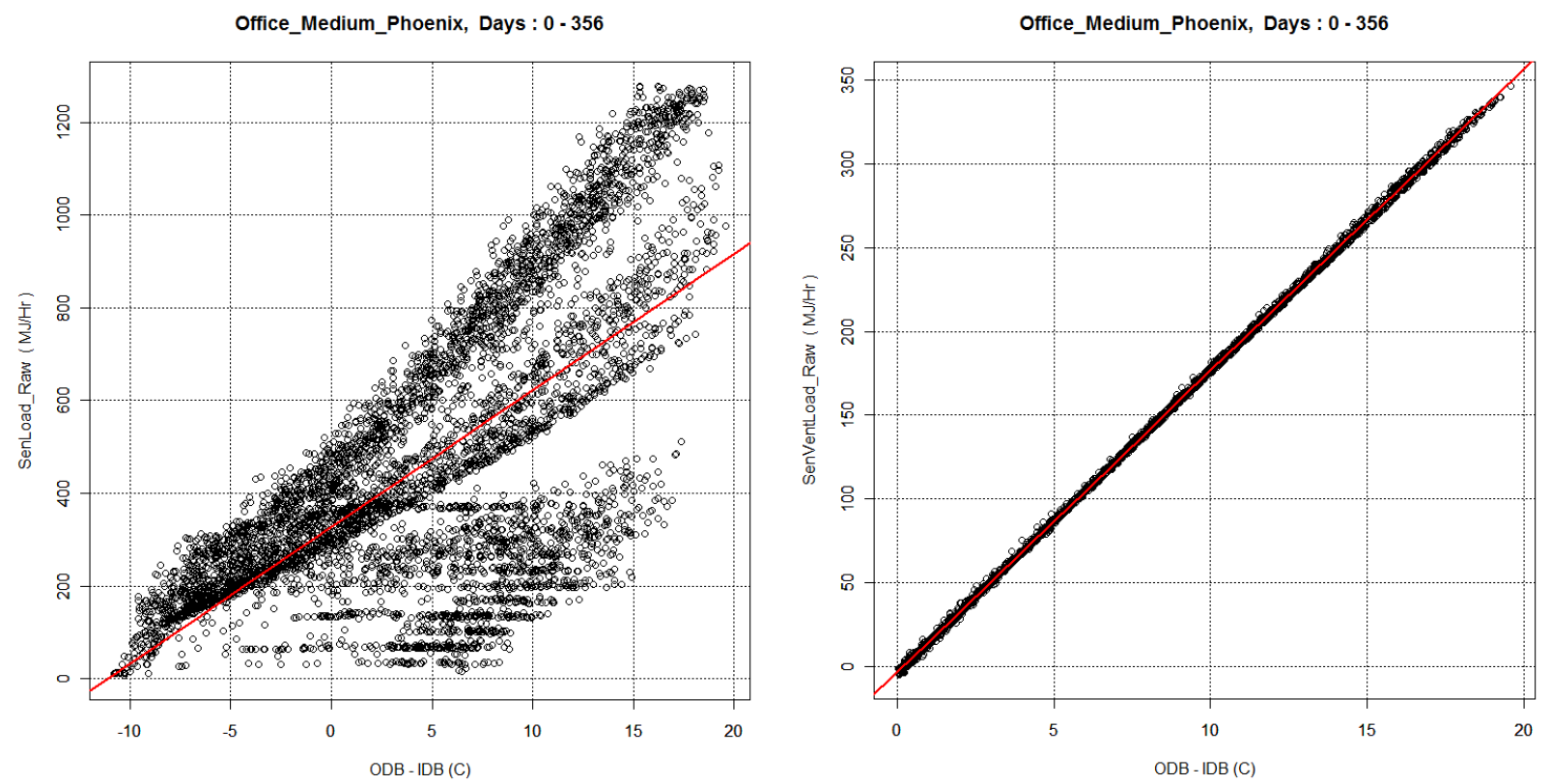

Figure A-18 Phoenix Medium Office building type with Phoenix weather. Raw sensible load as affected by envelope temperature differential. Left: sensible load (Intercept $=327.65 \mathrm{MJ} / \mathrm{h}$; Slope $=29.45 \mathrm{MJ} / \mathrm{hC})$. Right: sensible ventilation load $($ Slope $=18.01$; Slope Fraction $=$ $18.01 / 29.45=0.61)$.

Table A.10 Calculation of S\&I fraction in three cities using the Phoenix Medium Office building model with Phoenix weather.

\begin{tabular}{c|c|c} 
City & DT & $\begin{array}{c}\text { S\&I } \\
\text { Fraction }\end{array}$ \\
\hline SanFran & 0.8 & 0.93 \\
\hline Chicago & 7.5 & 0.60 \\
\hline Phoenix & 18.0 & 0.38
\end{tabular}




\section{A.4.4 Chicago Large Office}

The Large Office building type uses chillers, no DX, so this response model (Figure A-19 and Table A.11) was based on total loads. The intercept-to-slope ratio is $4250 / 410=10.36$, which is significantly smaller than values shown for Medium Office in Table A.4. This is evidence supporting the need to distinguish between these two versions of the Office building type.
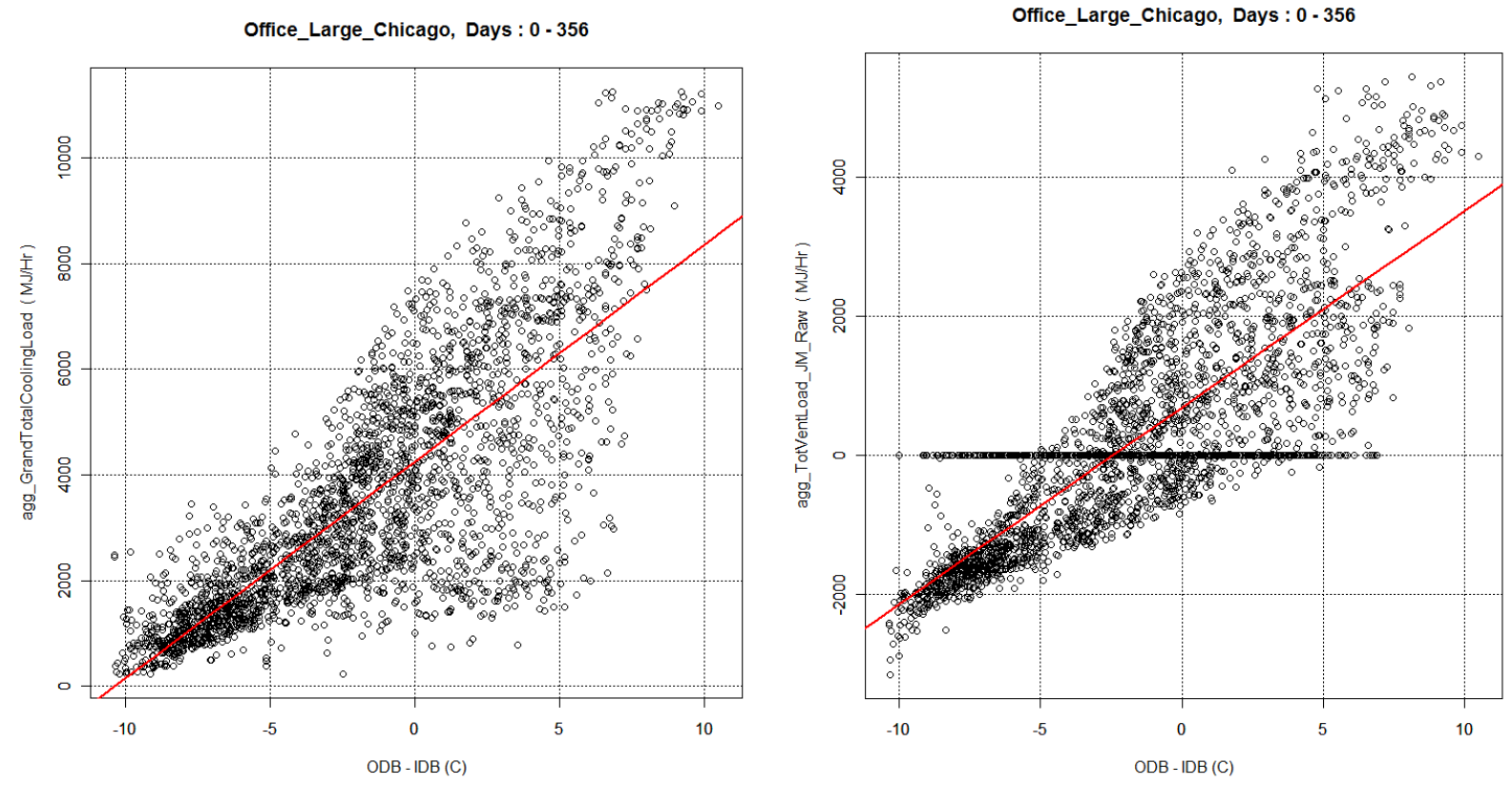

Figure A-19 Raw total load for the Chicago Large Office building type with Chicago weather as affected by envelope temperature differential. Left: total load (Intercept $=4250+/-30 \mathrm{MJ} / \mathrm{h}$; Slope $=410+/-6 \mathrm{MJ} / \mathrm{hC})$. Right: total ventilation load $($ Slope $=283+/-4$; Slope Fraction $=$ $0.69)$.

Table A.11 Calculation of S\&I fraction in three cities using the Chicago Large Office building model with Chicago weather.

\begin{tabular}{c|c|c} 
City & DT & $\begin{array}{c}\text { S\&I } \\
\text { Fraction }\end{array}$ \\
\hline SanFran & 0.8 & 0.93 \\
\hline Chicago & 7.5 & 0.58 \\
\hline Phoenix & 18.0 & 0.37
\end{tabular}




\section{A.4.5 Chicago Secondary School}

This is a comparison of the sensible and total load models for the Chicago version of the Secondary School building type. The sensible version is used in the RTUCC and reported in Table A.1.

\section{A.4.5.1 Sensible Loads}

The Secondary School building uses both DX and chiller. This sensible response model (Figure A-20) is based on the DX systems in the building and is the version reported in Table A.1.
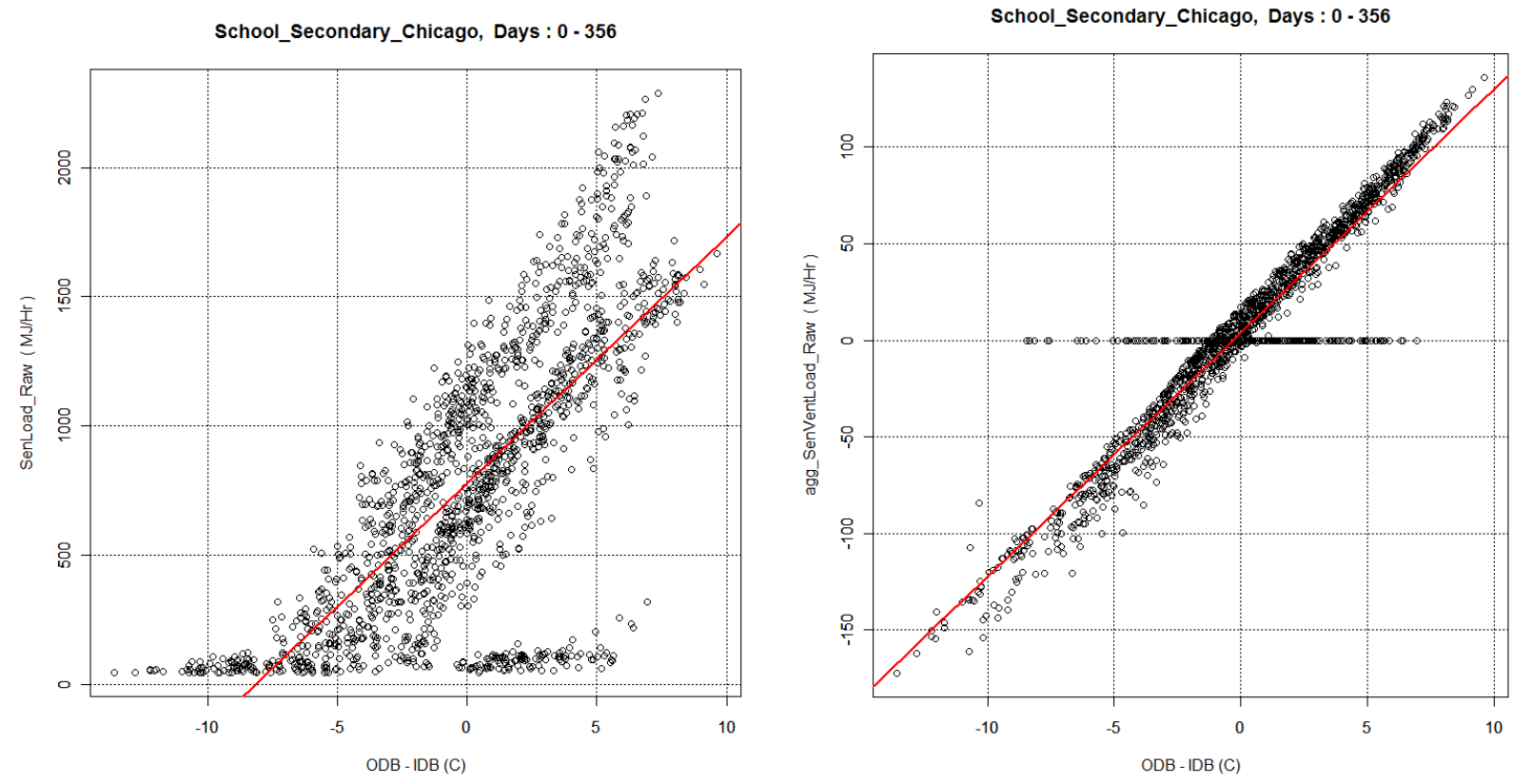

Figure A-20 Sensible load for the Secondary School building type. Left: sensible load $($ Intercept $=779+/-9 \mathrm{MJ} / \mathrm{h}$; Slope $=95+/-2 \mathrm{MJ} / \mathrm{hC} ; \mathrm{I} / \mathrm{S}=8.2 \mathrm{C})$. Right: sensible ventilation load $($ Slope $=12.62+/-0.10$; Slope Fraction $=0.13)$. 


\section{A.4.5.2 Total Loads}

The Secondary School building uses both DX and chiller. This total (= Sensible + Latent) model is based on both the DX and chiller systems in the building (Figure A-21). The I/S ratios here $(3256 / 339.1=9.6)$ is similar to the ratio $(779 / 95=8.0)$ which can be calculated in Section A.4.5.1, which is based only on the DX systems.
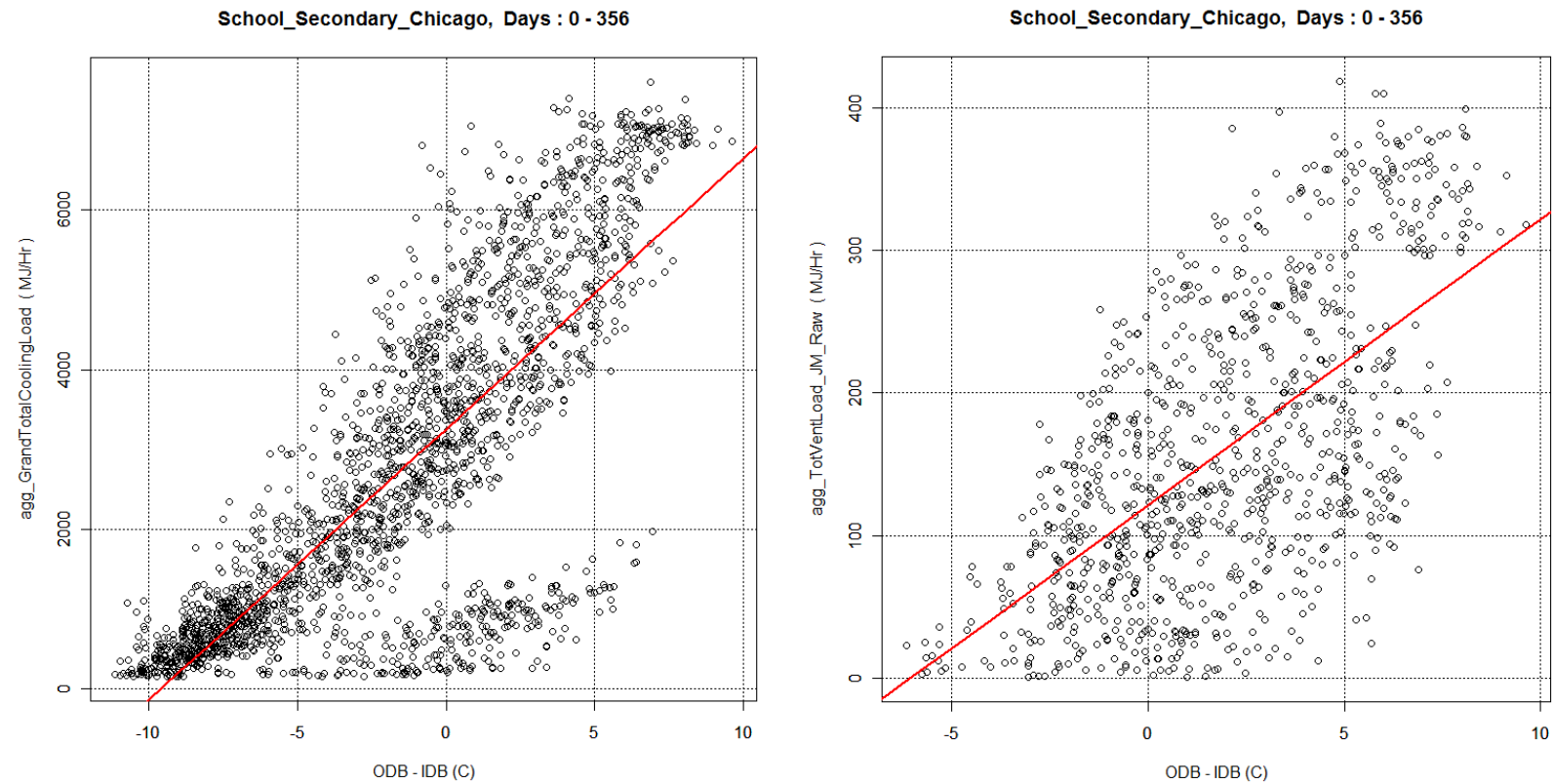

Figure A-21 Total load for the Secondary School building type. Left: total load (Intercept = $3256 \mathrm{MJ} / \mathrm{h}$; Slope $=339.1 \mathrm{MJ} / \mathrm{hC}$; I/S = 9.6C). Right: total ventilation load (Slope = 20.04; Slope Fraction $=0.06$ ). 


\section{A.5 Model Runs With Chicago Weather}

The results in sections A.5.1 through A.5.14 document the EnergyPlus runs that serve as the basis for each building response model. All of these building types have been simulated with Chicago weather. Response models are based on sensible-load predictions from EnergyPlus unless noted to be total-load data. Each response model (the red lines) has two components: a sensible load-line, and a sensible-ventilation load-line. In the sections that follow A.5.1, figures that are similar to Figure A-22 will use a more abbreviated caption showing mainly numerical results; refer back to Figure A-22 for the full explanation.

\section{A.5.1 Chicago Small OfFice}

EnergyPlus run results and response-model characteristics for the Chicago Small Office building type are shown in Figure A-22.
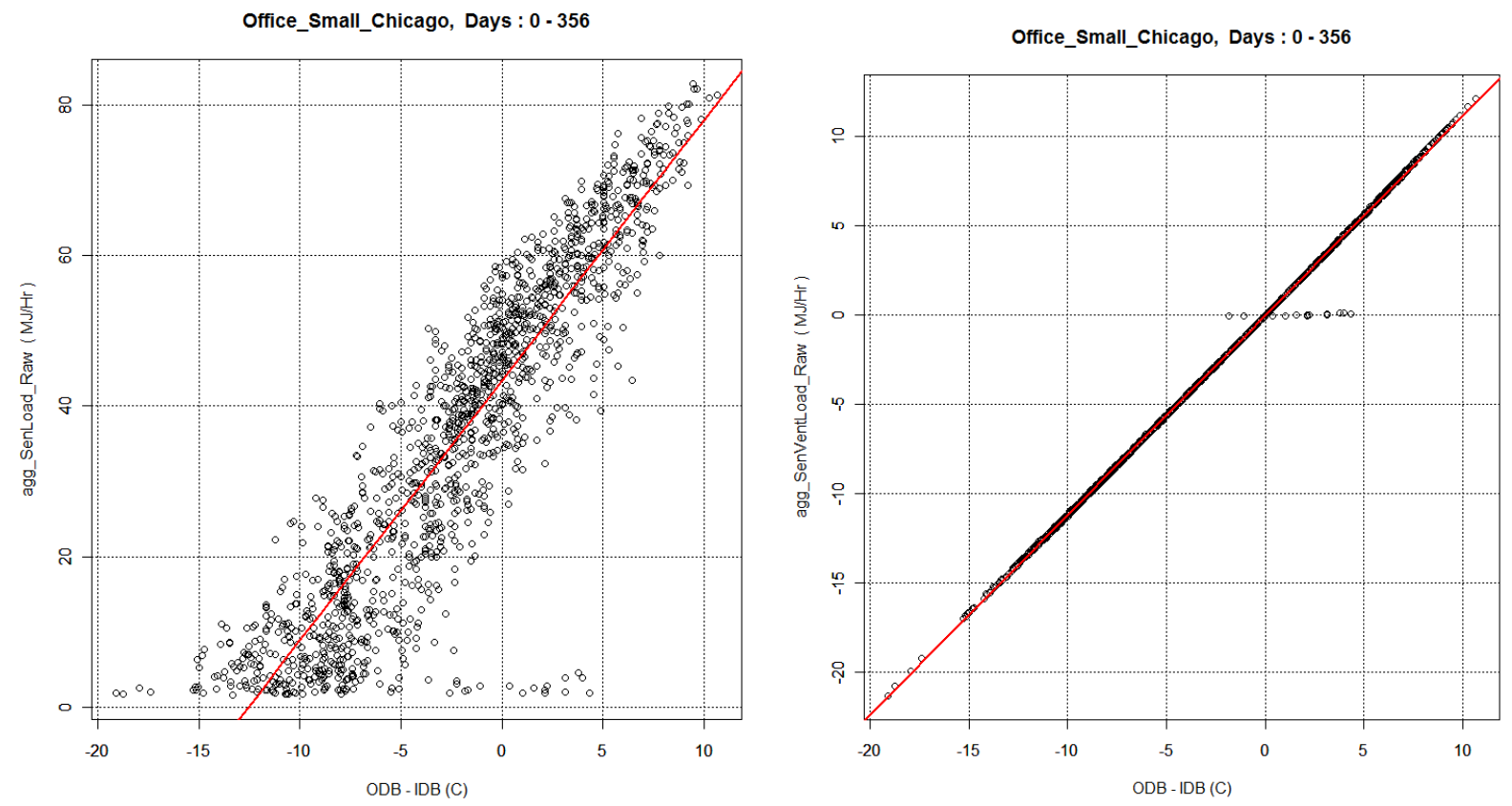

Figure A-22 Chicago Small Office building with Chicago weather. Raw sensible load as affected by envelope temperature differential. Left: sensible load (Intercept $=43.4+/-0.3 \mathrm{MJ} / \mathrm{h}$; Slope $=3.46+/-0.04 \mathrm{MJ} / \mathrm{hC})$. Right: sensible ventilation load (Slope $=1.120+/-0.001$; Slope Fraction $=0.32$ ).

\section{A.5.2 Chicago Medium OfFice}

See Section A.4.2.1 and Figure A-9. 


\section{A.5.3 Chicago Large OfFice}

See Section A.4.4 and Figure A-19.

\section{A.5.4 Chicago Primary School}

EnergyPlus run results and response-model characteristics for the Chicago Primary School building type are shown in Figure A-23.
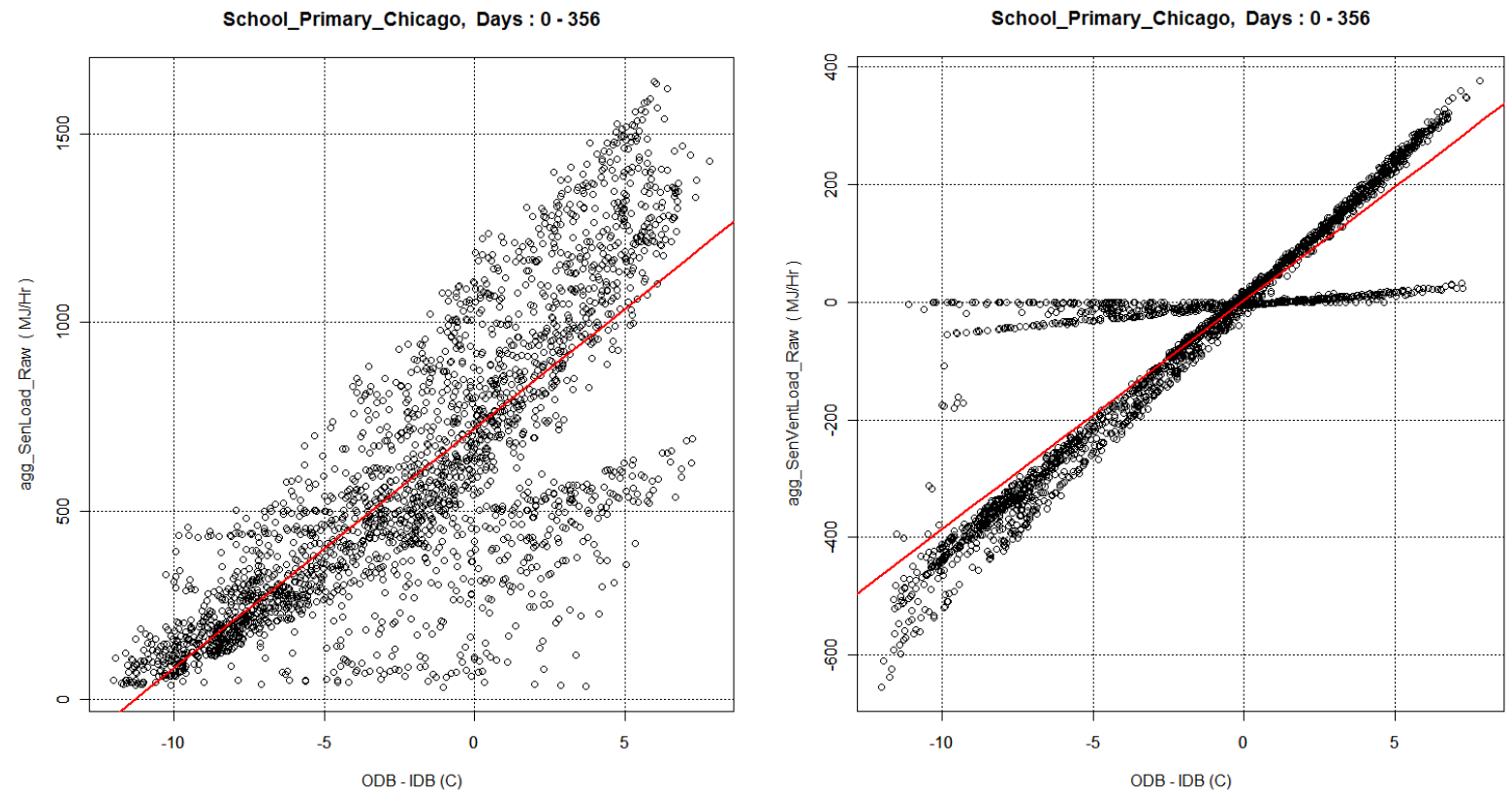

Figure A-23 Chicago Primary School. Left: sensible load (Intercept $=$ Intercept $=718+/-5$ $\mathrm{MJ} / \mathrm{h}$; Slope $=63.5+/-1.0 \mathrm{MJ} / \mathrm{hC}$ ). Right: sensible ventilation load (Slope = $38.8+/-0.3$; Slope Fraction $=0.61$ ).

\section{A.5.5 Chicago Secondary School}

See Section A.4.5.1 and Figure A-20. 


\section{A.5.6 WAREHOUSE}

This initial analysis shown in Figure A-24 indicates strong economizer effects for the Warehouse building type. It was re-analyzed in Figure A-25 to use only hours where the ventilation loads are positive.
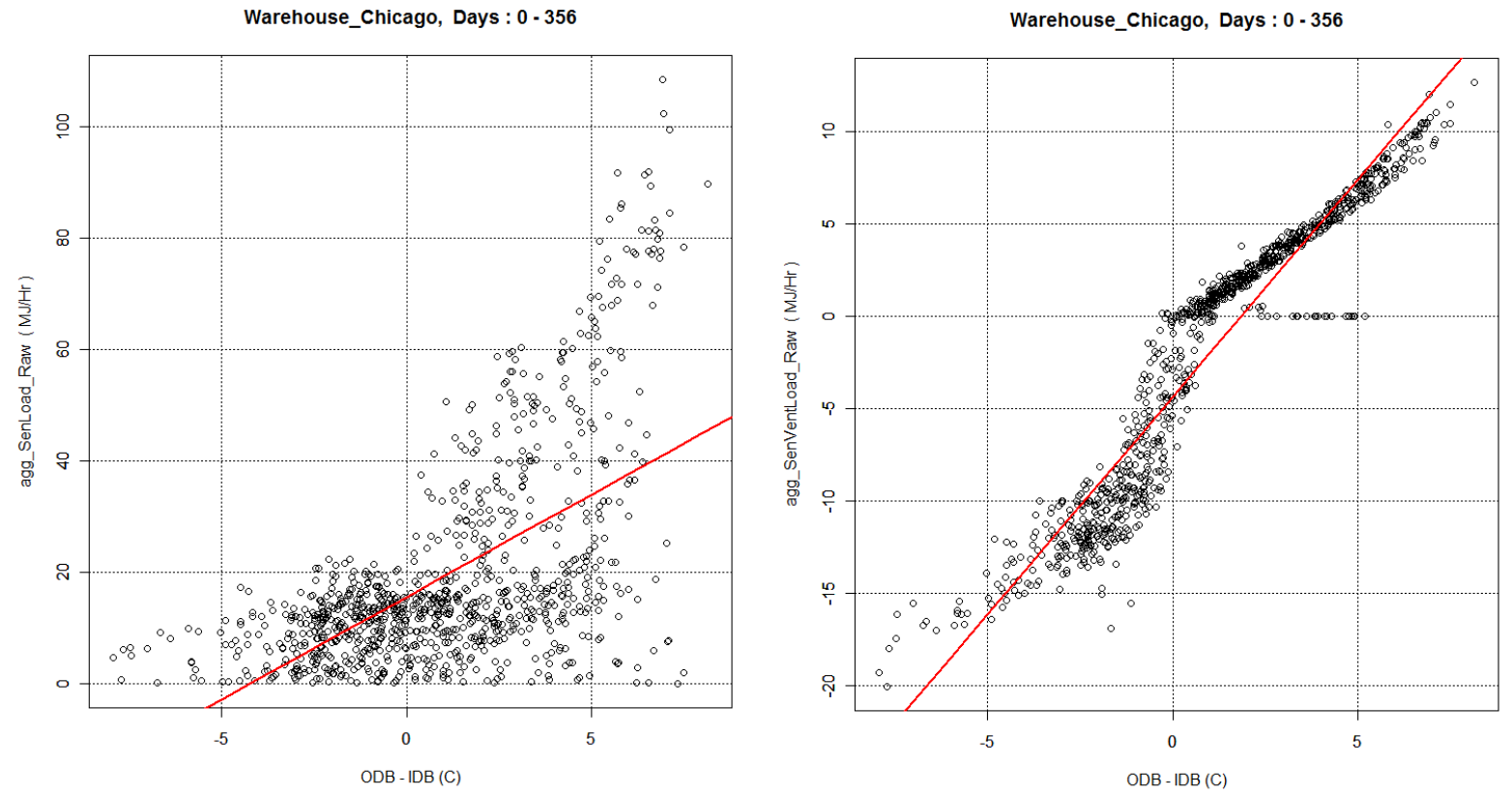

Figure A-24 Warehouse. Left: sensible load (Intercept $=15.5+/-0.5 \mathrm{MJ} / \mathrm{h}$; Slope $=3.68+/$ $0.16 \mathrm{MJ} / \mathrm{hC})$. Right: sensible ventilation load (Slope $=2.35+/-0.02$; Slope Fraction $=0.64$ ).

In Figure A-25 negative ventilation loads are excluded. This is done by filtering on the ventilation load data and then using the resulting set of hours in the sensible load plot. This still left some zero-load points. These were excluded in one more level of filtering, as shown in Figure A-26. These final results are the ones used in the RTUCC. 

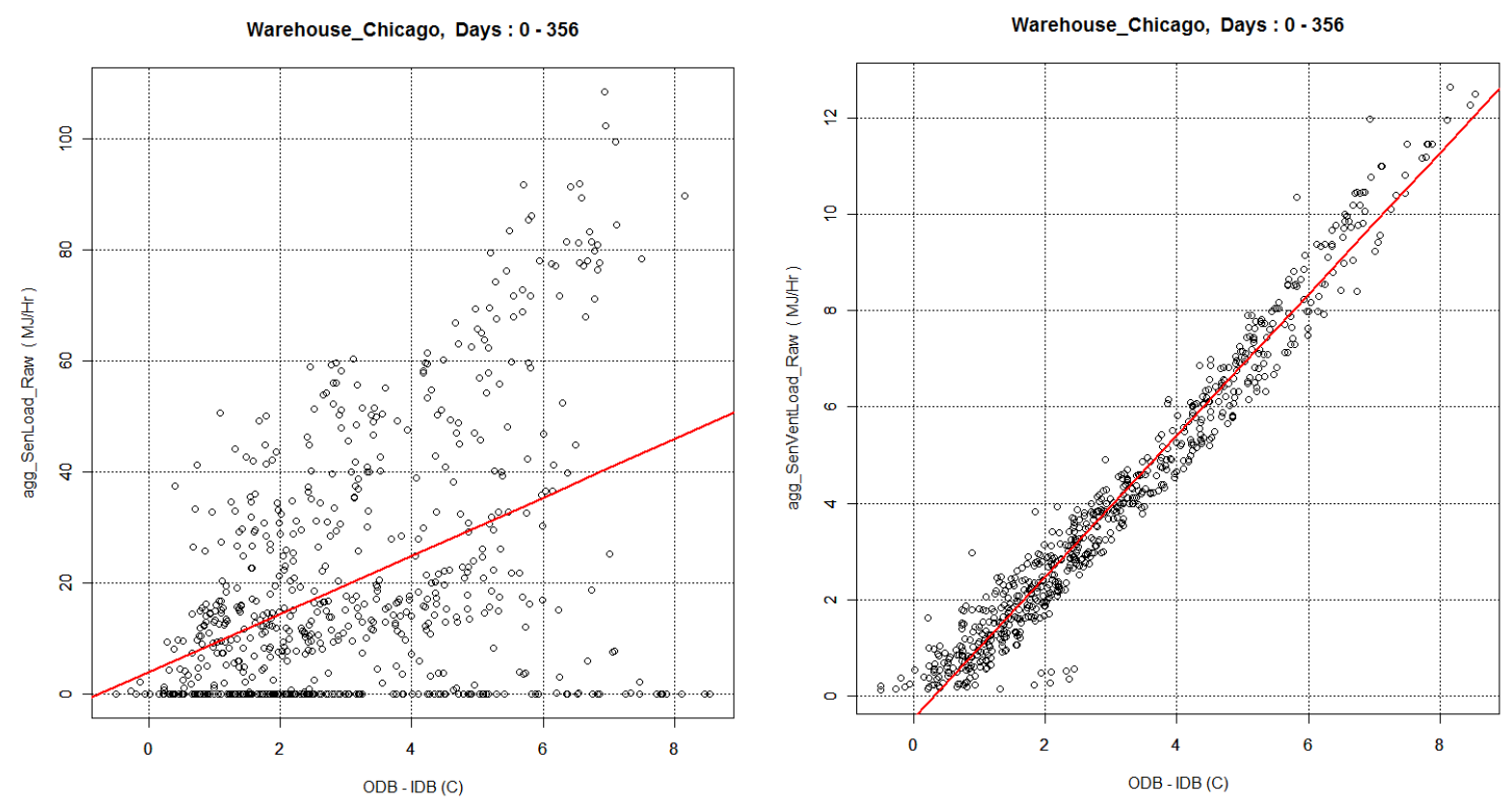

Figure A-25 Warehouse. Left: sensible load (Intercept $=3.8+/-1.5 \mathrm{MJ} / \mathrm{h}$; Slope $=5.3+/-0.4$ $\mathrm{MJ} / \mathrm{hC})$. Right: sensible ventilation load (Slope $=1.47$ +/- 0.01; Slope Fraction $=0.28)$.
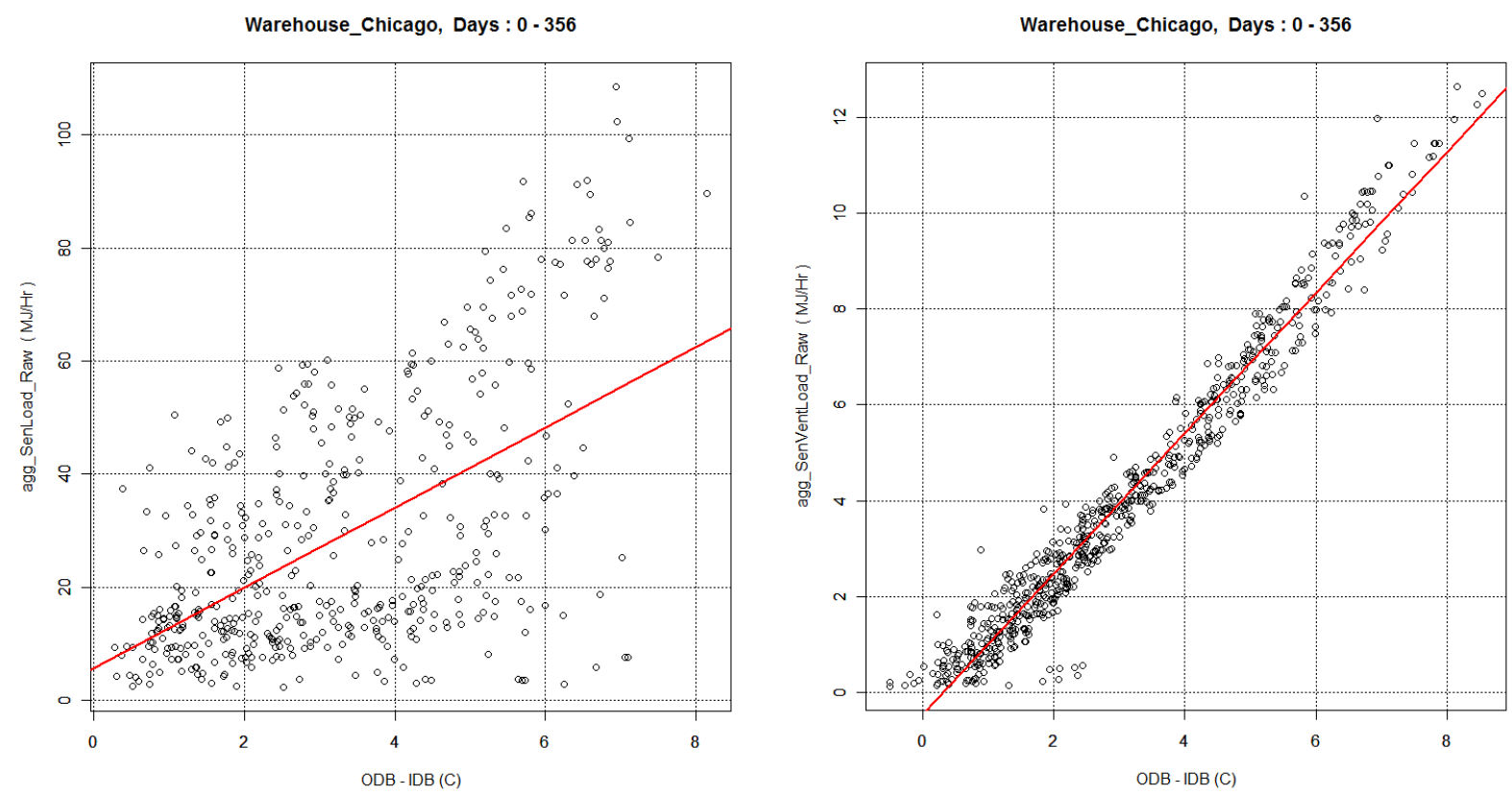

Figure A-26 Warehouse; hours with zero load are excluded. Left: sensible load (Intercept $=5.7$ +/- $1.7 \mathrm{MJ} / \mathrm{h}$; Slope =7.1 +/- 0.5 MJ/hC). Right: sensible ventilation load (Slope $=1.47$ +/0.01 ; Slope Fraction $=0.21$ ). 


\section{A.5.7 STAND-Alone RetaIL}

EnergyPlus run results and response-model characteristics for the Stand-Alone Retail building type are shown in Figure A-27.
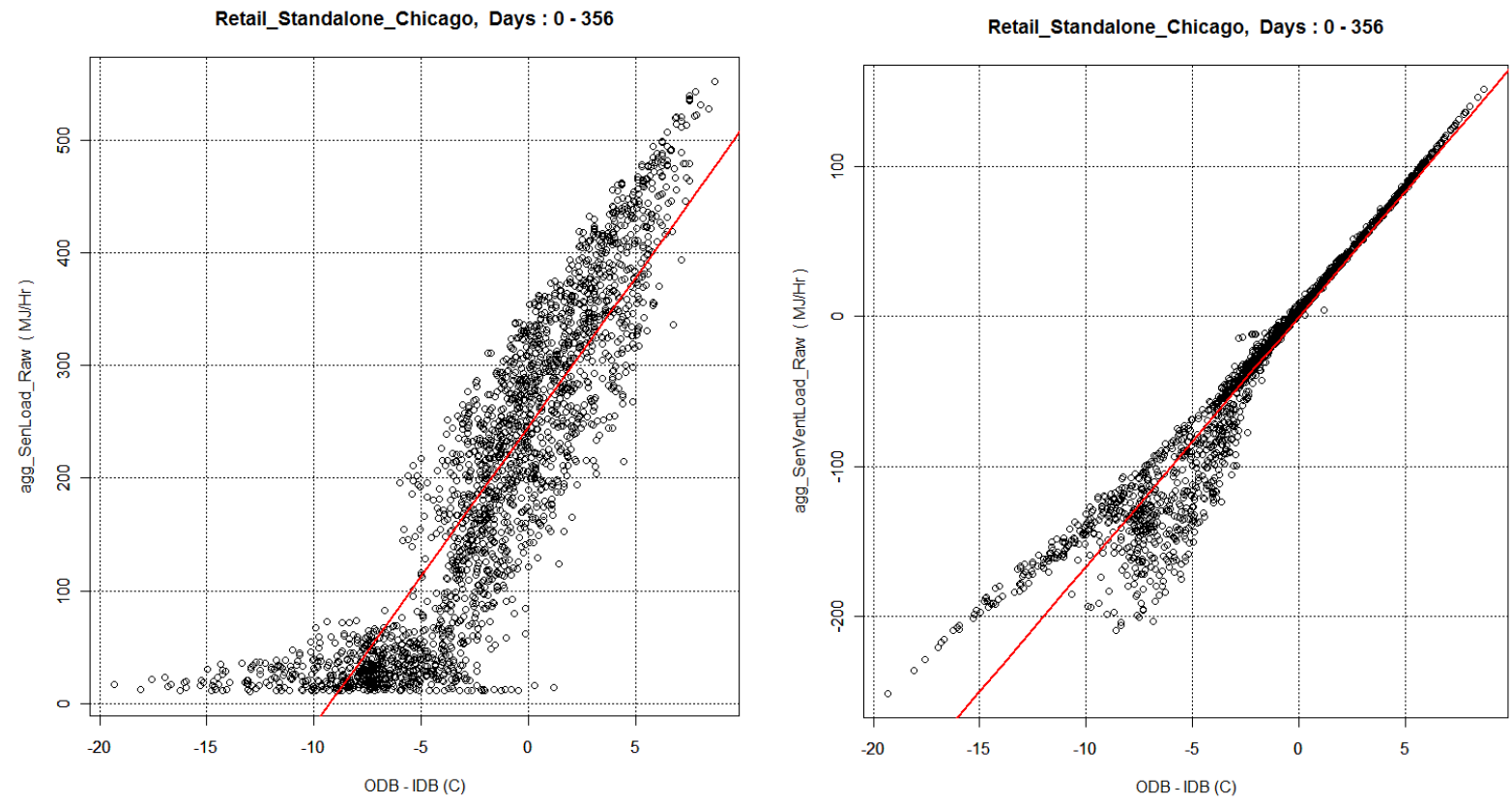

Figure A-27 Stand-Alone Retail. Left: sensible load (Intercept $=245.4+/-1.5 \mathrm{MJ} / \mathrm{h}$; Slope $=$ $26.5+/-0.3 \mathrm{MJ} / \mathrm{hC})$. Right: sensible ventilation load (Slope $=16.69+/-0.09$; Slope Fraction $=$ $0.63)$. 


\section{A.5.8 STRIP Mall Retail}

EnergyPlus run results and response-model characteristics for the Strip Mall Retail building type are shown in Figure A-28.
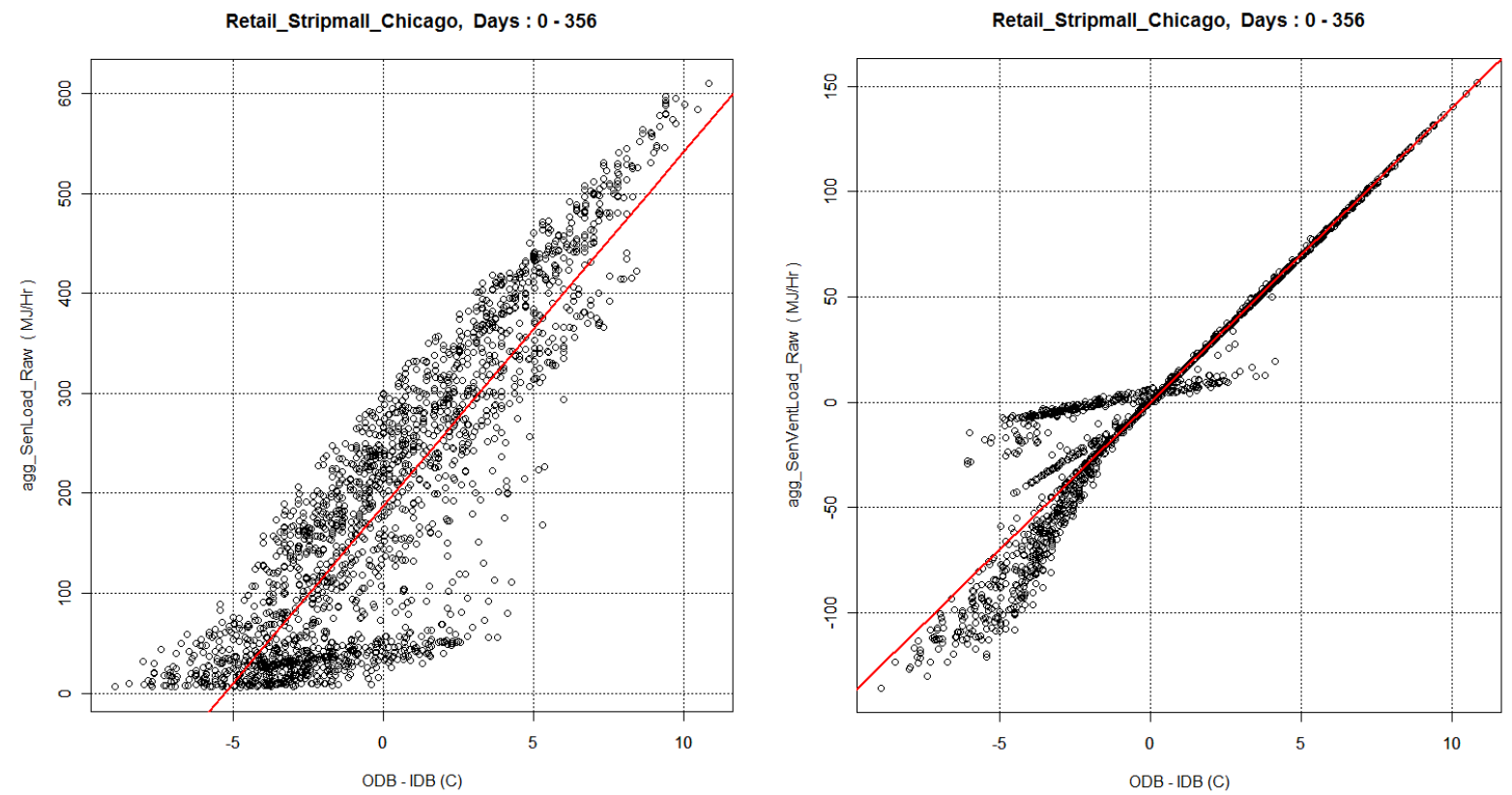

Figure A-28 Strip Mall Retail. Left: sensible load (Intercept $=187.2+/-1.6 \mathrm{MJ} / \mathrm{h}$; Slope $=35.4$ +/- 0.4 MJ/hC). Right: sensible ventilation load $($ Slope $=14.00$ +/- 0.09; Slope Fraction = 0.40). 


\section{A.5.9 Mid-Rise APARTMENT}

EnergyPlus run results and response-model characteristics for the Mid-Rise Apartment building type are shown in Figure A-29.

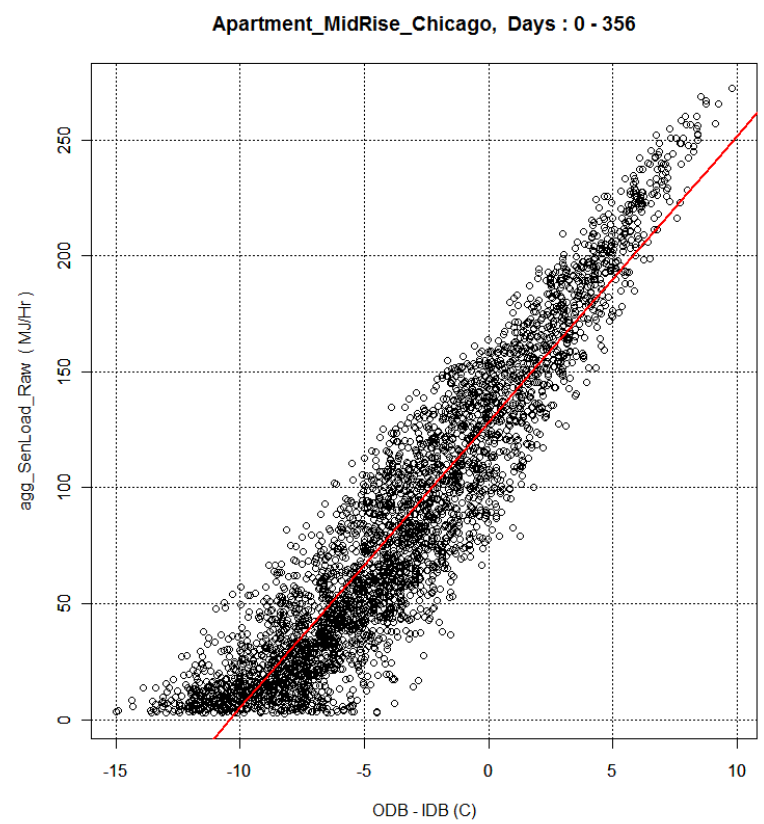

(Mechanical ventilation not reported.)

Figure A-29 Mid-Rise Apartment. Left: sensible load (Intercept $=128.3+/-0.5 \mathrm{MJ} / \mathrm{h}$; Slope = $12.32+/-0.08 \mathrm{MJ} / \mathrm{hC})$. Right: (none). Slope Fraction = NA (0.30 assumed). 


\section{A.5.10 Sit-Down Restaurant}

EnergyPlus run results and response-model characteristics for the Sit-Down Restaurant building type are shown in Figure A-30.
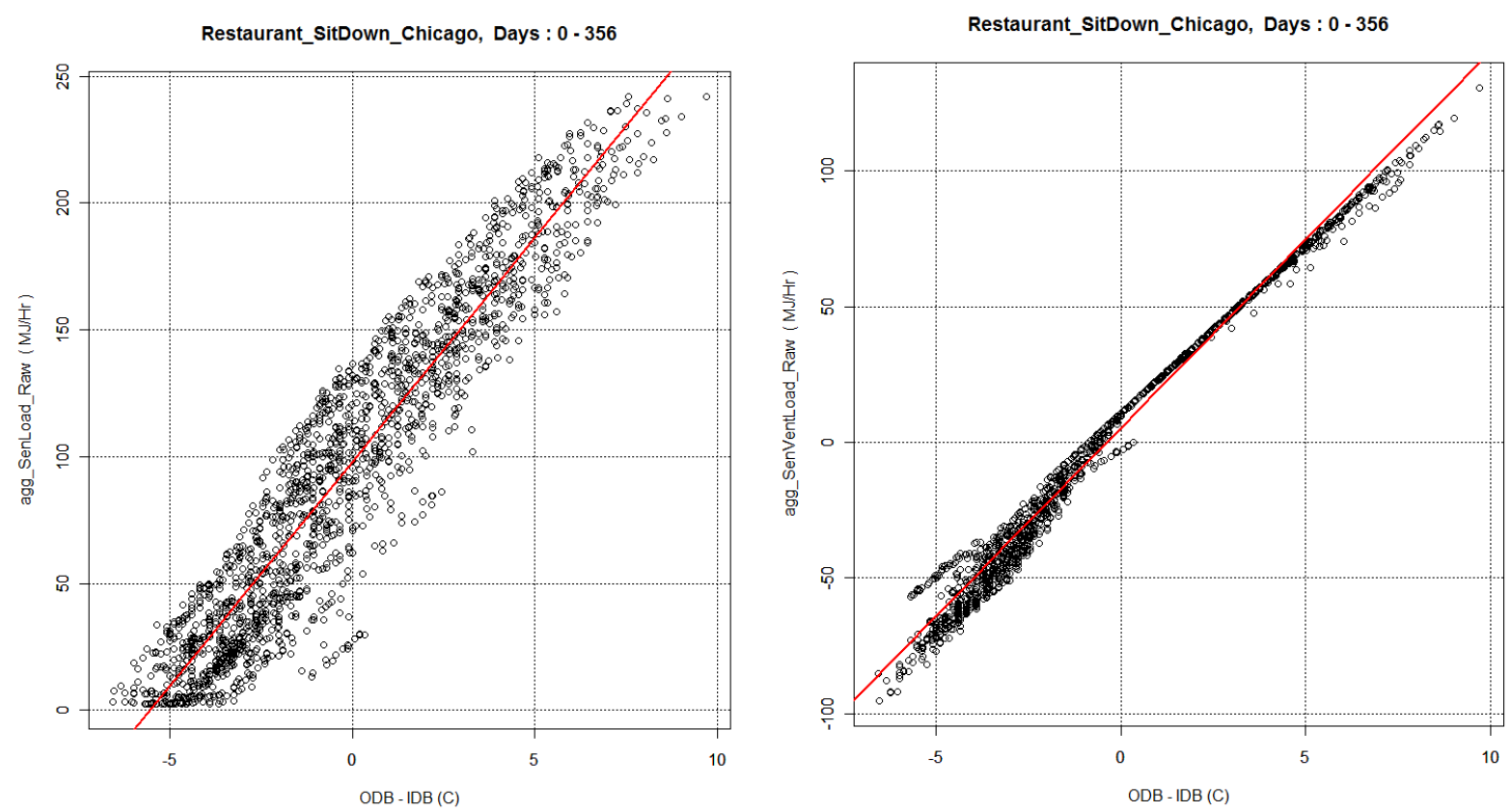

Figure A-30 Sit-Down Restaurant. Left: sensible load (Intercept $=98.0+/-0.5 \mathrm{MJ} / \mathrm{h}$; Slope $=$ $17.65+/-0.14 \mathrm{MJ} / \mathrm{hC}$ ). Right: sensible ventilation load (Slope = $13.88+/-0.04$; Slope Fraction $=0.79$ ).

\section{A.5.11 FAST-FoOd RESTAURANT}

See Section A.4.1.1 and Figure A-4. 


\section{A.5.12 LARgE Hotel}

EnergyPlus run results and response-model characteristics for the Large Hotel building type are shown in Figure A-31.
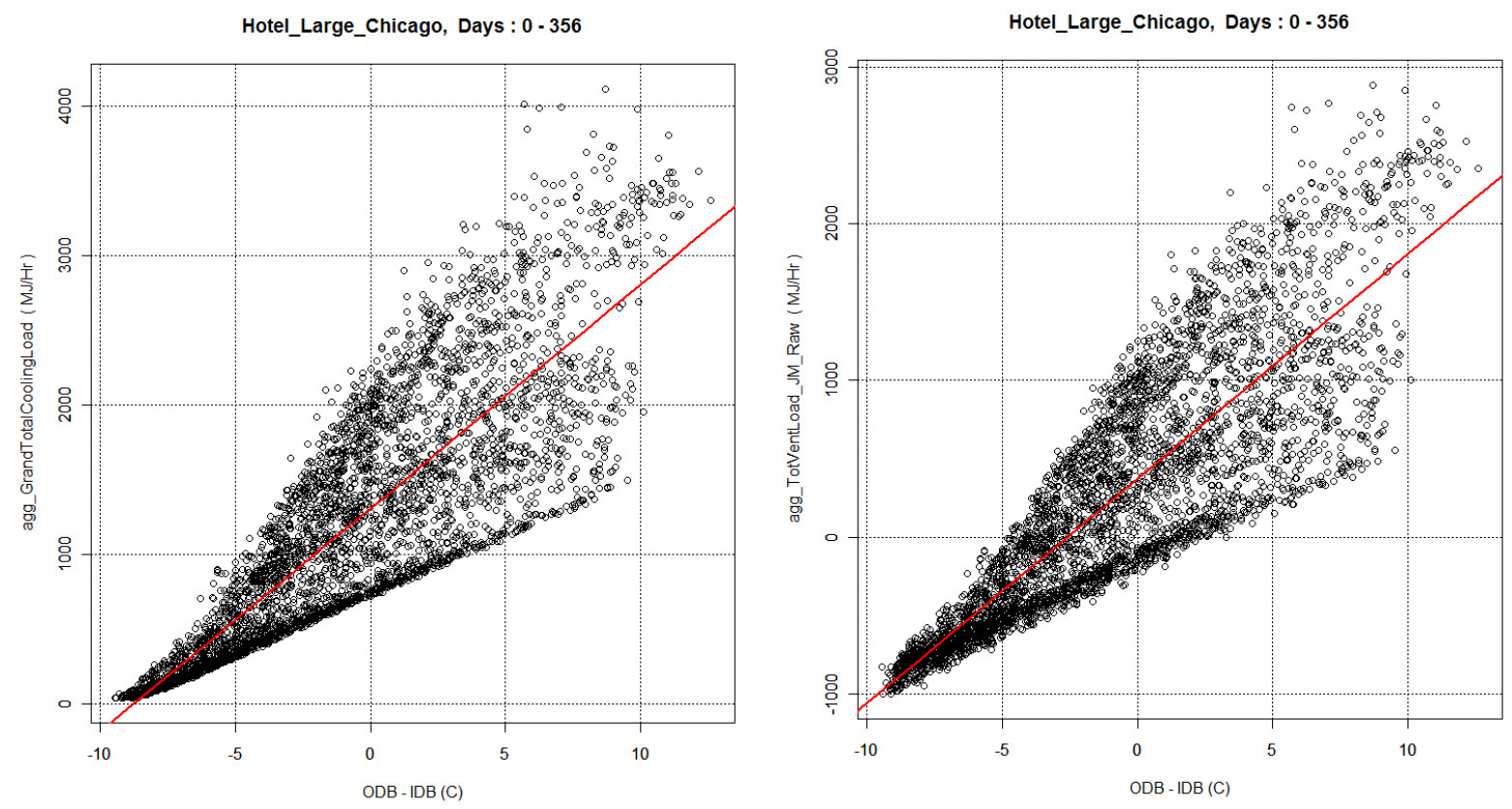

Figure A-31 Large Hotel. Left: sensible load (Intercept $=1313+/-7 \mathrm{MJ} / \mathrm{h}$; Slope $=149.5+/$ 1.4 MJ/hC). Right: sensible ventilation load (Slope $=143.3+/-1.3$; Slope Fraction $=0.96$ ). 


\section{A.5.13 Hospital HealthCare}

EnergyPlus run results and response-model characteristics for the Hospital Healthcare building type are shown in Figure A-32.

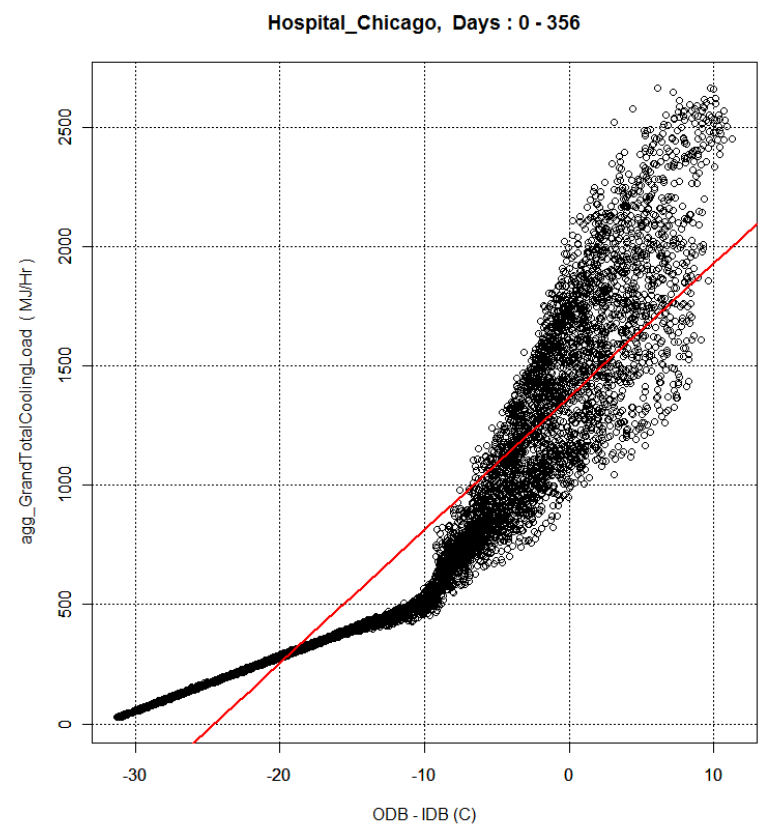

(Ventilation loads not reported)

Figure A-32 Hospital Healthcare. Left: sensible load (Intercept $=1370+/-4 \mathrm{MJ} / \mathrm{h}$; Slope $=$ $55.8+/-0.3 \mathrm{MJ} / \mathrm{hC})$. Right: (none). Slope Fraction = NA (0.8 assumed). 


\section{A.5.14 OutPatient HealthCare}

EnergyPlus run results and response-model characteristics for the Outpatient Healthcare building type are shown in Figure A-33.
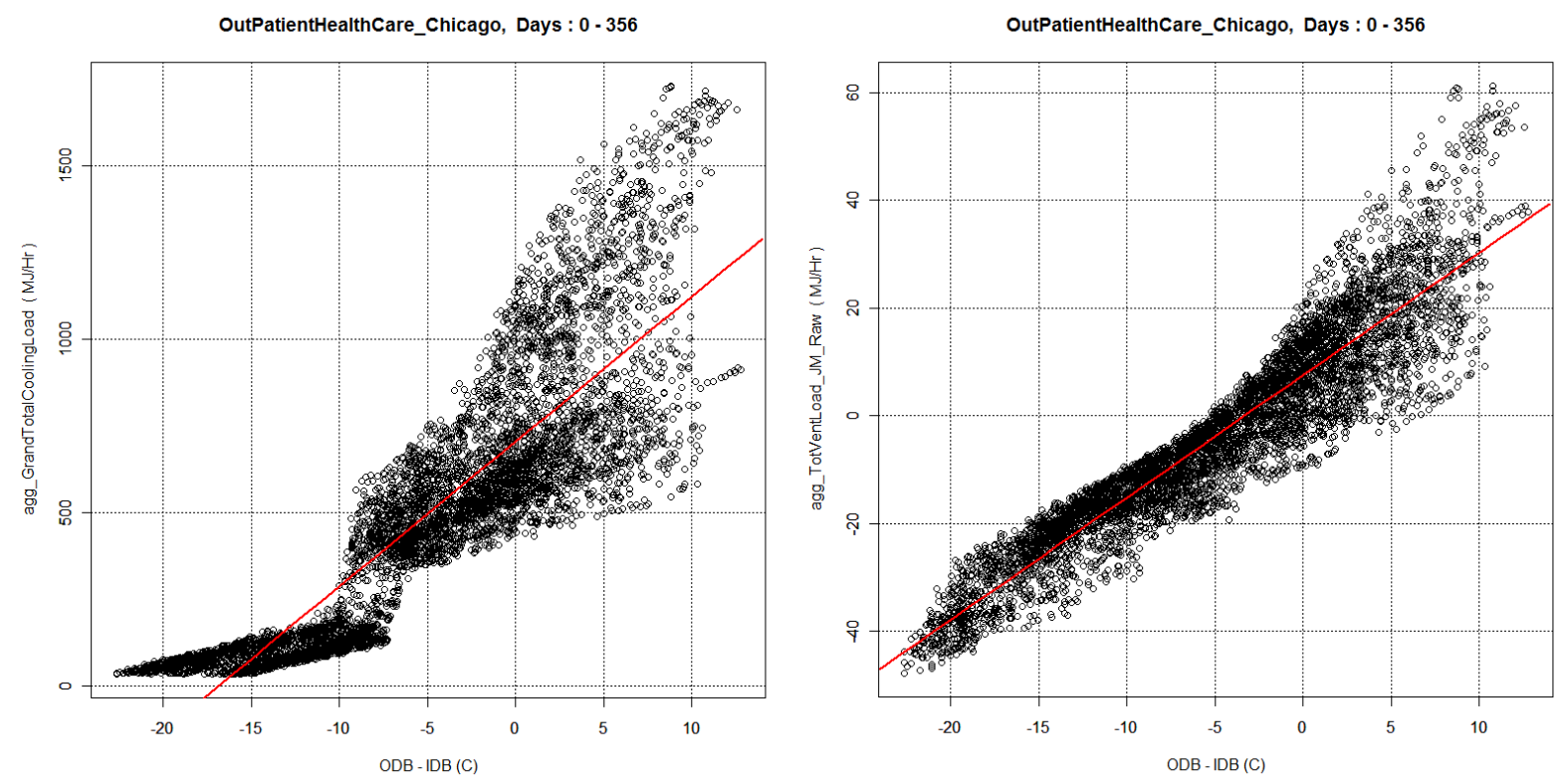

Figure A-33 Outpatient Healthcare. Left: sensible load (Intercept $=704+/-3 \mathrm{MJ} / \mathrm{h}$; Slope $=$ $41.8+/-0.3 \mathrm{MJ} / \mathrm{hC}$ ). Right: sensible ventilation load (Slope $=2.270+/-0.011$; Slope Fraction = $0.054)$. 


\section{A.6 Check of Total Ventilation Load Calculation}

The plots in Figure A-34 are a comparison check between the total-ventilation loads calculated in the post processing and the ventilation loads reported by EnergyPlus in the Zone.Mechanical.Ventilation.Cooling.Load.Increase variable. There is primarily one-to-one agreement in the Sit-Down Restaurant data (right plot). EnergyPlus documentation does not specify if their ventilation variable is sensible or total; agreement here indicates it must be total. A similar sensible variable is not available in EnergyPlus. For this reason, ventilation loads were calculated wherever sensible loads were used to establish the building model.
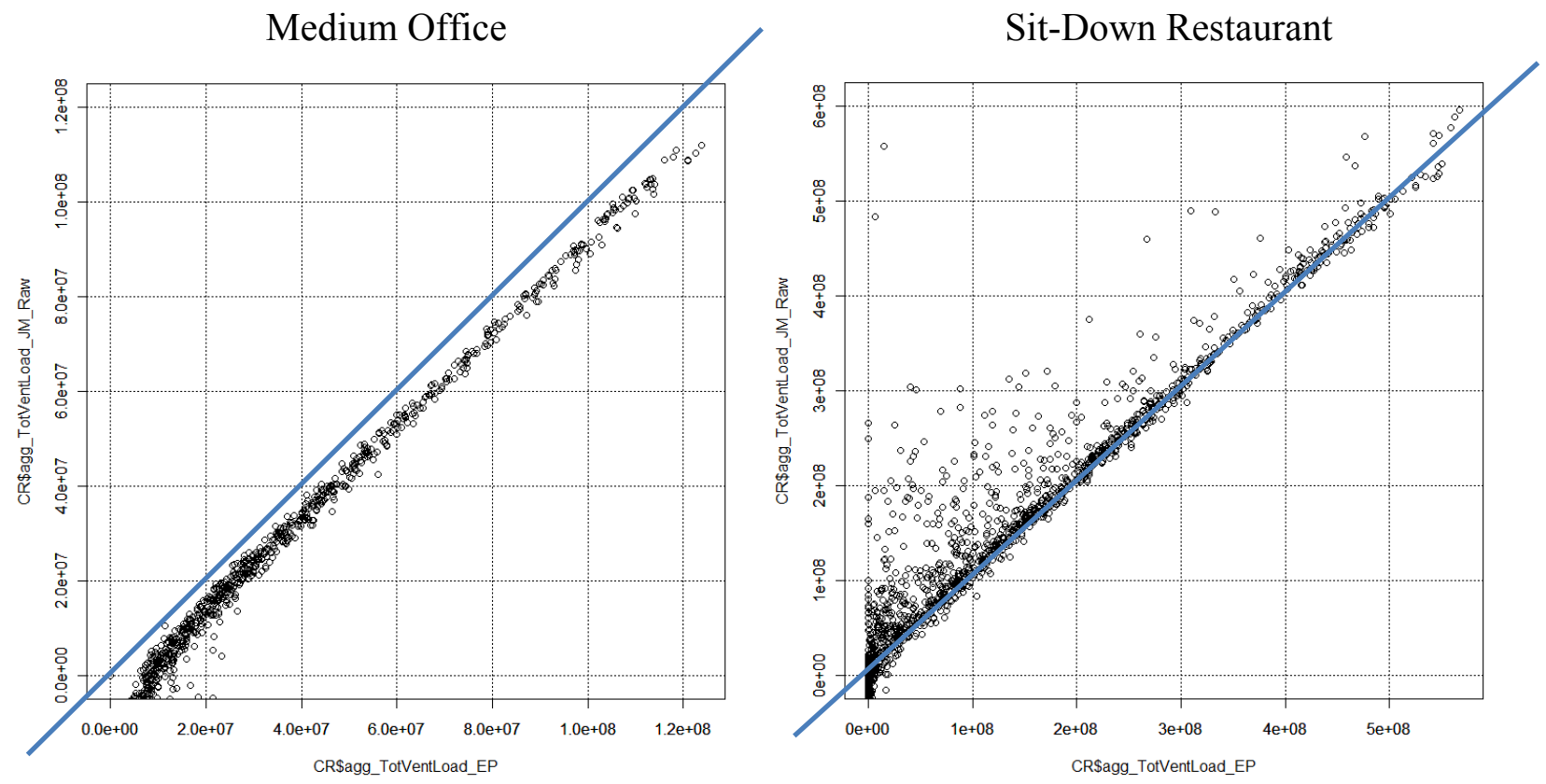

Figure A-34 Comparison of the calculated total-ventilation load (calculated in post processing using ventilation mass flow) and reported ventilation load (load reported directly from EnergyPlus). The blue line indicates where data would be expected if there was one-to-one agreement. Left: Medium Office. Right: Sit-Down Restaurant. 


\section{A.7 HuMidity-RATio DifFERENCE}

The plots in Figure A-35 illustrate the difference between the outside and inside humidity ratio throughout the year, as indexed by temperature differential. This shows a positive humidity-ratio difference during the summer cooling season and a negative difference in the winter. Summer cooling and the resulting dehumidification acts to suppress the indoor humidity ratio. This is shown here for future reference in support of developing an indoor humidity-ratio model based on EnergyPlus runs.
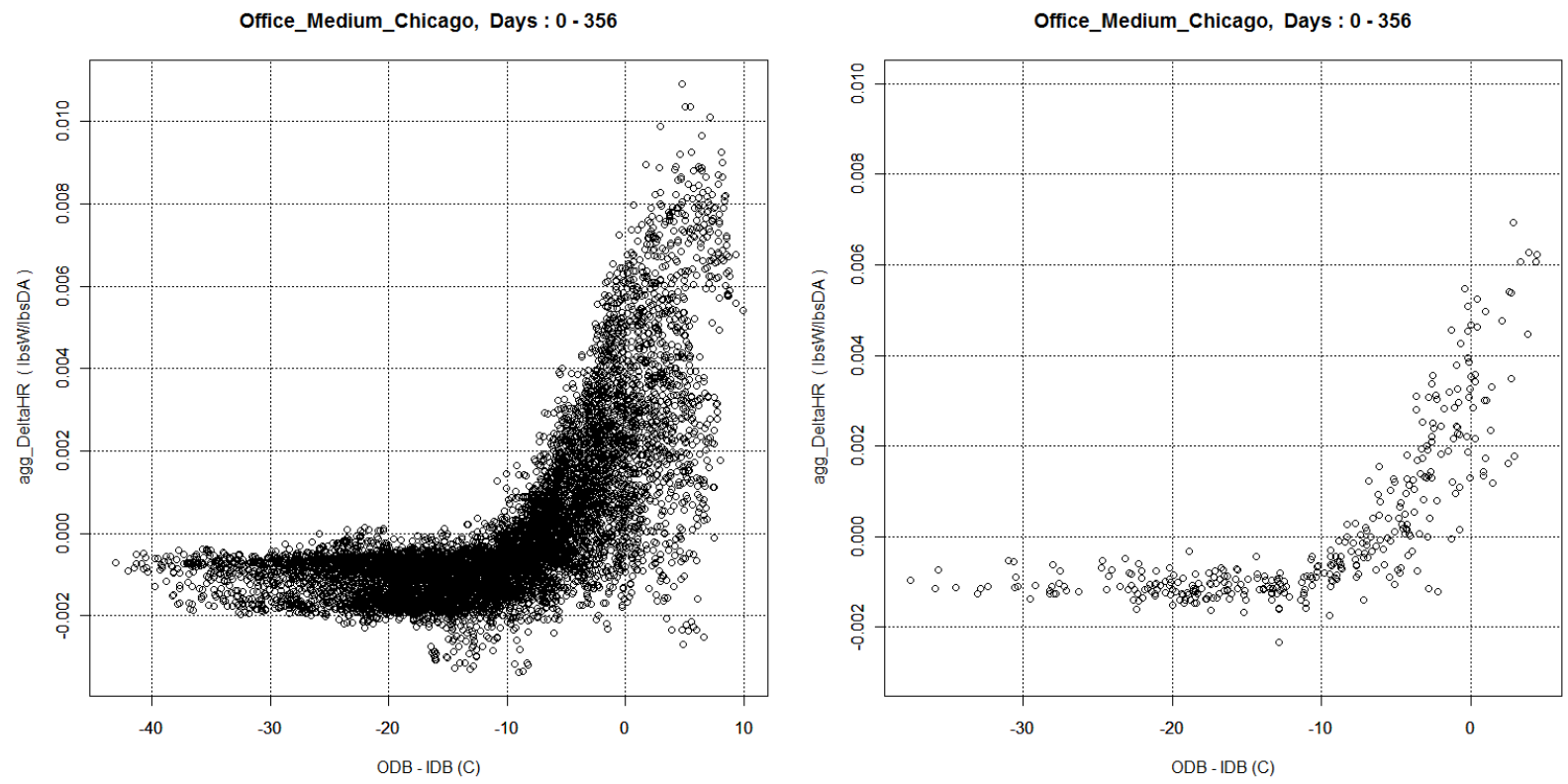

Figure A-35 Humidity-ratio differential (outdoor minus indoor) as affected by temperature differential (outdoor minus indoor). Left: raw hourly data. Right: daily averages. 


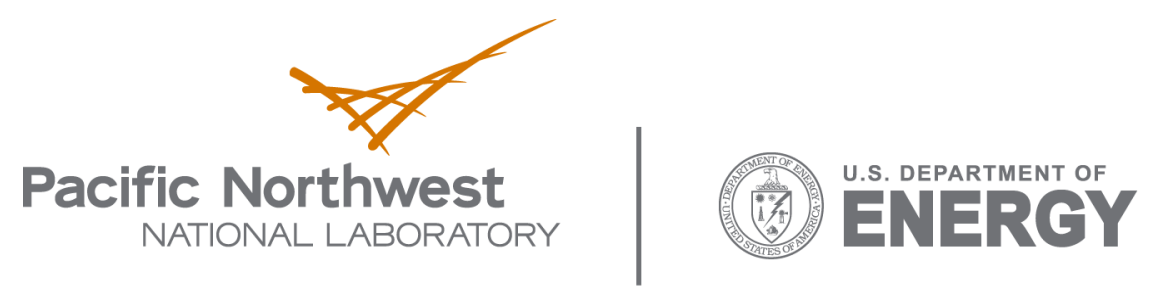

902 Battelle Boulevard

P.O. Box 999

Richland, WA 99352

1-888-375-PNNL (7665)

www.pnl.gov 